\title{
Hadronization model tuning in GENIE v3
}

Júlia Tena-Vidalø, ${ }^{1, *}$ Costas Andreopoulos, ${ }^{1,2}$ Christopher Barry, ${ }^{1}$ Steve Dennis, ${ }^{1, \dagger}$ Steve Dytman, ${ }^{3}$ Hugh Gallagher, ${ }^{4}$ Steven Gardiner, ${ }^{5}$ Walter Giele, ${ }^{5}$ Robert Hatcher, ${ }^{5}$ Or Hen, ${ }^{6}$ Igor D. Kakorin, ${ }^{7}$ Konstantin S. Kuzmin, ${ }^{7,8}$ Anselmo Meregaglia, ${ }^{9}$ Vadim A. Naumov, ${ }^{7}$ Afroditi Papadopoulou, ${ }^{6}$ Marco Roda $\odot,{ }^{10}$ Vladyslav Syrotenko, ${ }^{4}$ and Jeremy Wolcott ${ }^{4}$

(GENIE Collaboration)

\author{
${ }^{1}$ University of Liverpool, Department of Physics, Liverpool, England L69 7ZE, United Kingdom \\ ${ }^{2}$ Science and Technology Facilities Council, Rutherford Appleton Laboratory, \\ Particle Physics Department, Oxfordshire, England OX11 OQX, United Kingdom \\ ${ }^{3}$ University of Pittsburgh, Deptartment of Physics and Astronomy, Pittsburgh, Pennsylvania 15260, USA \\ ${ }^{4}$ Tufts University, Department of Physics and Astronomy, Medford, Massachusetts 02155, USA \\ ${ }^{5}$ Fermi National Accelerator Laboratory, Batavia, Illinois 60510, USA \\ ${ }^{6}$ Massachusetts Institute of Technology, Department of Physics, Cambridge, Massachusetts 02139, USA \\ ${ }^{7}$ Joint Institute for Nuclear Research (JINR), Dubna, Moscow region 141980, Russia \\ ${ }^{8}$ Alikhanov Institute for Theoretical and Experimental Physics (ITEP) of NRC "Kurchatov Institute", \\ Moscow 117218, Russia \\ ${ }^{9}$ CENBG, Université de Bordeaux, CNRS/IN2P3, 33175 Gradignan, France \\ ${ }^{10}$ University of Liverpool, Department of Physics, Liverpool, England L69 7ZE, United Kingdom
}

(Received 8 September 2021; accepted 30 November 2021; published 24 January 2022)

\begin{abstract}
The GENIE neutrino Monte Carlo describes neutrino-induced hadronization with an effective model, known as Andreopoulos-Gallagher-Kehayias-Yang (AGKY), which is interfaced with PYTHIA at high invariant mass. Only the low-mass AGKY model parameters were extracted from hadronic shower data from the FNAL $15 \mathrm{ft}$ and BEBC experiments. In this paper, the first hadronization tune on averaged charged multiplicity data from deuterium and hydrogen bubble chamber experiments is presented, with a complete estimation of parameter uncertainties. A partial tune on deuterium data highlights the tensions between hydrogen and deuterium datasets.
\end{abstract}

DOI: 10.1103/PhysRevD.105.012009

\section{INTRODUCTION}

The next generation of neutrino oscillation experiments will rely on the precise understanding of neutrino interactions at the percent level. Experiments such as T2K [1], NOvA [2], MINERvA [3], and MicroBooNE [4] study neutrino interactions over a broad energy range. In the few $\mathrm{GeV}$ region, $0 \pi$ and $1 \pi$ contributions dominate the event rate. Hence, most of the effort has been focused on the theoretical understanding of these interactions [5-9] as well as the precise measurement of quasielastic [10-16] and pion production cross sections [10,17-23]. Pions, before

\footnotetext{
publications@genie_mc.org

${ }^{\dagger}$ Present address: University of Cambridge.
}

Published by the American Physical Society under the terms of the Creative Commons Attribution 4.0 International license. Further distribution of this work must maintain attribution to the author(s) and the published article's title, journal citation, and DOI. Funded by SCOAP ${ }^{3}$. final-state interactions (FSIs), can be produced by either neutrino resonance interactions [24] or hadronization processes. Hadronization models provide information about the multiplicities and kinematics of the hadrons before FSI given the neutrino-nucleon interaction and the event kinematics. The knowledge of the exact mixture of hadrons in showers affects the efficiency to distinguish between neutral-current (NC) and charged-current (CC) events, the event topological characterization $[11,20]$, impacts the estimation of backgrounds [25], and the calorimetric energy reconstruction. FSI modeling and detector efficiency corrections are also crucial to avoid confusion in measurements of neutrino-induced hadron production. Unfortunately, due to the lack of unified models for exclusive hadronic multiparticle production over the energy range of interest for neutrino experiments, one must resort to stitching together different modeling ingredients. The GENIE neutrino Monte Carlo (MC) event generator [26] uses the Andreopoulos-Gallagher-KehayiasYang (AGKY) hadronization model [27] whose validity 

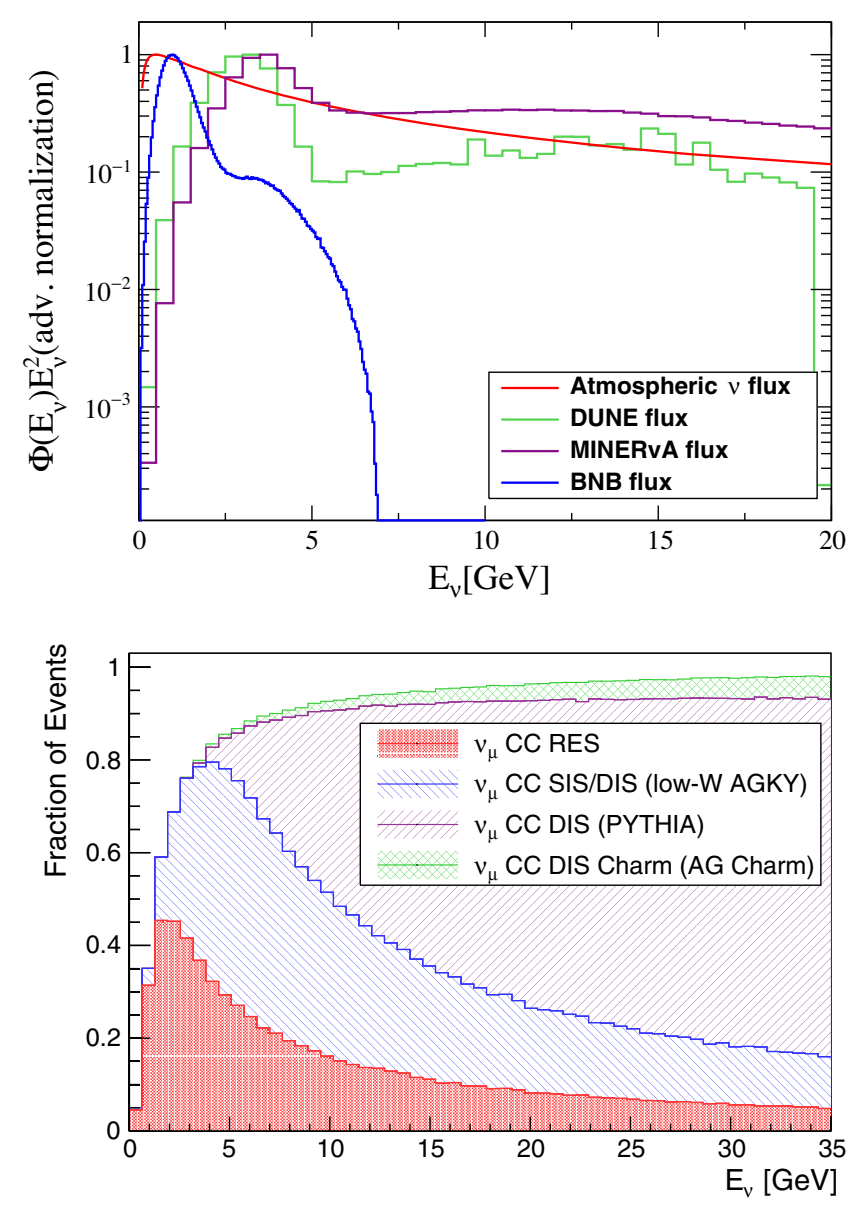

FIG. 1. Normalized neutrino fluxes are shown for the atmospheric neutrino flux at Kamioka [33], DUNE [34], MINERvA [35], and BNB [36] flux predictions (top plot). Breakdown of CC events as a function of the neutrino energy from $\nu_{\mu}$ scattering on ${ }^{40} \mathrm{Ar}$ (bottom plot). The plot was obtained with GENIE v3.00.06 using tune G18_02a_02_11a. The main components are resonance (RES), shallow and deep inelastic scattering (SIS/DIS), and deep inelastic charm production (DIS Charm). DIS contributions are split according to the hadronization model used: low- $W$ AGKY and PYTHIA.

extends down to the inelastic threshold. At low hadronic invariant mass $W$, the model is based on the Koba-NielsenOlesen scaling law (KNO), while at high $W$, it is based on the PYTHIA MC [28].

Current and future experiments operate at high energies, where potential biases originating from hadronization mismodeling become important. For instance, DUNE [29], IceCube-Gen2 [30,31], and ORCA [32] will focus on the 2 to $20 \mathrm{GeV}$ energy range where deep inelastic scattering (DIS) events are dominant. The neutrino energy dependence on the main inelastic components of the expected event rate for $\mathrm{CC} \nu_{\mu}{ }^{40} \mathrm{Ar}$ scattering is shown in Fig. 1. Some relevant neutrino fluxes of interest are shown in Fig. 1 (top). It is seen that the contribution to the event rate from $\mathrm{GeV}$ neutrinos is mainly driven by $\mathrm{CC}$ resonant (RES) events as well as shallow inelastic scattering (SIS) and DIS events from the low-W AGKY model, whereas PYTHIA events dominate at high neutrino energies.

The description of the AGKY hadronization model implementation in GENIE is described in Sec. II. There is a separate hadronization model to simulate DIS charm production, the Aivazis, Olness, and Tung model [37]. Hadronic remnants produced in the interaction are hadronized with PYTHIA.

The AGKY model parameters controlling hadronization at low invariant masses were extracted from some of the FNAL $15 \mathrm{ft}$ bubble chamber and the Big European Bubble Chamber (BEBC) analyses [38,39]. PYTHIA has never been tuned to low-energy neutrino-induced hadronization data. In 2010, GENIE revisited the AGKY parameter values and modified a number of PYTHIA parameters using information from the NUX PYTHIA tune [40], as discussed in Sec. II. We refer to this parameter set as the 2010 GENIE AGKY tune or 2010 GENIE tune. Despite the modifications, several discrepancies between the model and neutrino-induced hadron shower data remained [41,42].

This paper summarises the results of the first tune of the AGKY hadronization model against averaged charged multiplicity data on hydrogen and deuterium targets from bubble chamber experiments. The analysis is performed within the GENIE v3.00.06 global analysis framework [24]. The base configuration used for all the plots presented here is the G18_02a_02_11a. This paper is organized as follows. The AGKY model specifics relevant for this work are described in Sec. II, followed by an explanation of the analysis procedure applied to the hydrogen and deuterium datasets in Sec. III. Section V discusses the free parameters in the model, and Sec. VI presents the construction of the likelihood function used for fitting. The AGKY best-fit results are summarized in Sec. VII.

\section{AGKY MODEL}

The AGKY [27] model is the main hadronization model used in GENIE. As a function of hadronic invariant mass $W$, three different regimes are defined: an empirical model anchored to bubble chamber data at low $W\left(W<W_{\text {min }}^{\text {tr }}\right)$, a pure PYTHIA region for high $W\left(W>W_{\max }^{\mathrm{tr}}\right)$, and a transition region that connects them. In the transition region, the probability to produce a PYTHIA event increases linearly with $W$, from zero at $W_{\min }^{\mathrm{tr}}$ to 1 at $W_{\max }^{\mathrm{tr}}$. The values of the transition region limits are $W_{\min }^{\mathrm{tr}}=2.3 \mathrm{GeV} / c^{2}$ and $W_{\max }^{\mathrm{tr}}=3.0 \mathrm{GeV} / c^{2}$. The empirical low- $W$ model and PYTHIA are valid in different mass ranges, and they are combined accordingly.

The low- $W$ AGKY and PYTHIA algorithms are described in the Secs. II A and II B, respectively. The contribution of the main inelastic components as a function of $W$ for events generated with the DUNE flux [34] is shown in Fig. 2. Most of the DIS/SIS events use the low- $W$ AGKY model, 


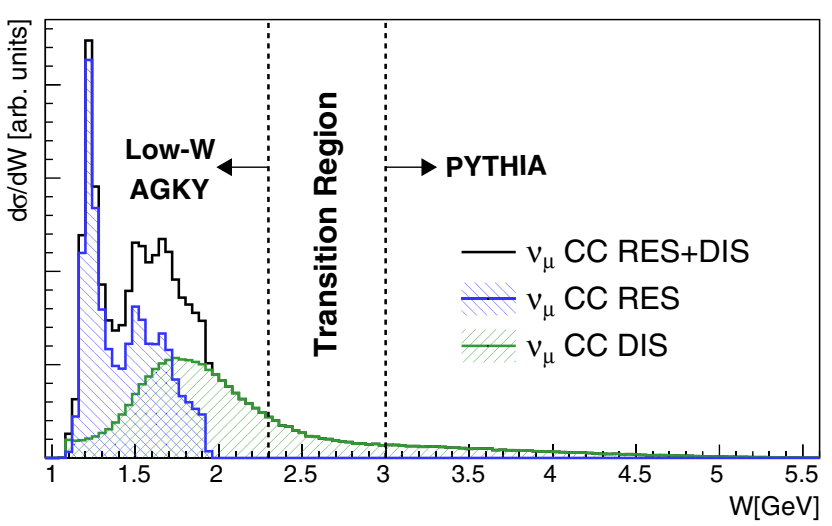

FIG. 2. Flux integrated CC inelastic differential cross section as a function of the hadronic invariant mass for a DUNE $\nu_{\mu}$ beam on ${ }^{40} \mathrm{Ar}$, obtained with the G18_02a_02_11a tune. The distribution is decomposed in RES and DIS contributions. The DIS contribution to the total number of events is $38 \%$ and $36 \%$ for RES events. The $\nu_{\mu}$ flux maximum is between 1 and $5 \mathrm{GeV}$.

while the PYTHIA events are coming from the high-energy tail of the beam.

\section{A. Effective low- $W$ AGKY hadronization model}

At low $W$, the showers are made of one baryon and any number of $\pi$ or $K$ consistent with momentum, charge, baryon and strange number, isospin and parity conservation laws:

$$
\begin{aligned}
& \nu_{\mu}+p \rightarrow \mu^{-}+X^{++}, \\
& \nu_{\mu}+n \rightarrow \mu^{-}+X^{+}, \\
& \bar{\nu}_{\mu}+p \rightarrow \mu^{+}+X^{0}, \\
& \bar{\nu}_{\mu}+n \rightarrow \mu^{+}+X^{-} .
\end{aligned}
$$

For instance, when approaching the pion production threshold, the $\nu_{\mu} p$ interaction would produce a shower made of a proton and a $\pi^{+}$. In general, the hadron multiplicity at the lowest possible $W$ is 2 as the hadronic final state can only be made of a pion and a nucleon.

As $W$ increases, more possibilities are available. The model draws random integer numbers from the simulated hadronic multiplicity distribution to generate the number of particles in the shower; then, the particles are labeled so that baryon number, charge, and strangeness are conserved. The particle content of a shower is selected so that the total mass is not exceeding $W$. The 4-momenta of the hadronic shower particles are generated by a weighted phase space decay of a particle of mass $W$ to the selected hadronic-multiparticle state. There are many ingredients in the simulation of the hadronic probability distribution: average hadronic multiplicity data, the KNO scaling law, particle content rules, phase space weighting, and others, as discussed in detail in Ref. [27]. In this paper, we focus on the description of the hadronic multiplicity. The hadronic multiplicity probability distribution depends on two ingredients: the measured average as a function of $W$, and an empirical parametrization of multiplicity dispersion. Both parametrizations must be extracted from data.

Empirical observation suggests that the average charged multiplicity is linear with $\ln W^{2}$ :

$$
\left\langle n_{\mathrm{ch}}\right\rangle(W)=\alpha_{\mathrm{ch}}+\beta_{\mathrm{ch}} \ln \left(\frac{W^{2}}{\mathrm{GeV}^{2} / c^{4}}\right) .
$$

The coefficients $\alpha_{\mathrm{ch}}$ and $\beta_{\mathrm{ch}}$ depend on the initial state, and their values can be extracted from neutrino-induced hadronization data; see Sec. III. This behavior has also been proven to be true for heavier nuclear targets $[43,44]$. From fits to $\pi^{0}$ production data, it is known that $\left\langle n_{\mathrm{ch}}\right\rangle \sim 0.5\left\langle n_{\pi^{0}}\right\rangle$ [45]. Therefore, the total hadronic multiplicity is obtained from the charged one as

$$
\langle n\rangle(W) \equiv 1.5\left\langle n_{\mathrm{ch}}\right\rangle(W) .
$$

Given the average $\langle n\rangle$, the hadronic multiplicity distribution, $n$, can be obtained from the KNO scaling law, which relates the dispersion of hadron multiplicities with a universal scaling function [46],

$$
\langle n\rangle P(n)=f\left(\frac{n}{\langle n\rangle}\right) .
$$

The scaling function $f(n /\langle n\rangle)$ is parametrized with the Levy function $L(n /\langle n\rangle ; \mathrm{c})$,

$$
L(n /\langle n\rangle ; \mathrm{c})=\frac{2 e^{-\mathrm{c}} \mathrm{c}^{\mathrm{c} \frac{n}{\langle n\rangle}+1}}{\Gamma\left(\mathrm{c} \frac{n}{\langle n\rangle}+1\right)},
$$

where $\Gamma$ is the gamma function and $\mathrm{c}$ is the free parameter that has to extracted from data and depends on the interaction isospin. By construction, the dispersion of the hadronic multiplicity distribution is independent from the average; see Fig. 3. The 2010 GENIE AGKY values of $\alpha_{\mathrm{ch}}$, $\beta_{\mathrm{ch}}$, and $\mathrm{c}$ are specified in Table I.

\section{B. PYTHIA in GENIE}

The PYTHIA algorithm is well known for its wide use in high-energy collider experiments to simulate the evolution from a few-body hard process to a multihadronic final state $[28,47]$. The PYTHIA hadronization model is based on the Lund string fragmentation framework, which describes the hadronization process as breakups in a string throughout production of new $q \bar{q}$ pairs [48]. Each string represents a color flux which is subject to a linear confined potential. In the Lund model, the $q \bar{q}$ pairs break by tunneling, which, together with causality, defines the Lund symmetric fragmentation function,

$$
f(z) \propto \frac{(1-z)^{a}}{z} \exp \left(\frac{-b m_{\perp}^{2}}{z}\right),
$$




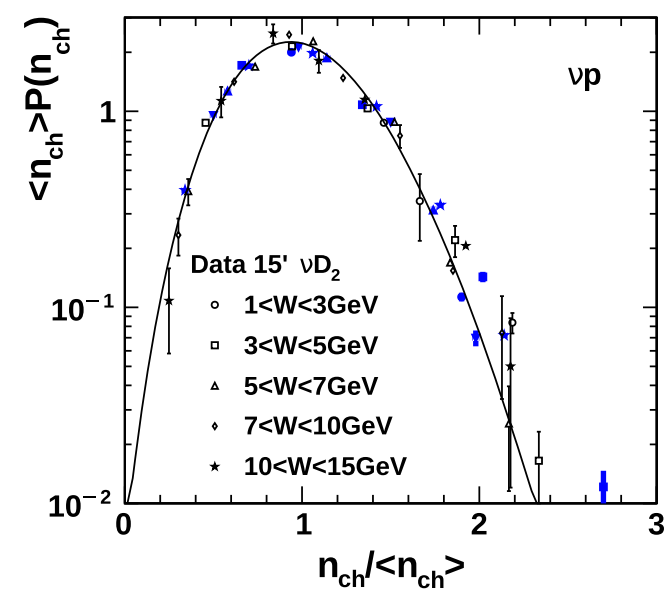

(a)

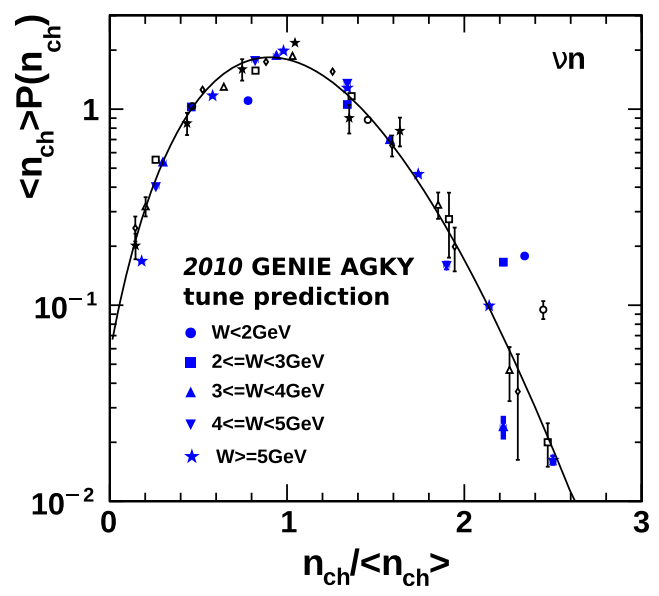

(b)

FIG. 3. KNO scaling distributions for neutrino interactions on deuterium [27]. The solid line is the best-fit result of the Levy function to FNAL $15 \mathrm{ft}$ bubble chamber data [38]. Blue dots show the 2010 GENIE AGKY prediction for a given $W$ range specified in the legend. (a) KNO distribution for $\nu p$ interactions and (b) KNO distribution for $\nu n$ interactions.

TABLE I. 2010 GENIE tune low- $W$ AGKY parameters.

\begin{tabular}{lcrcc}
\hline \hline Parameter & $\nu_{\mu} p$ & \multicolumn{1}{c}{$\nu_{\mu} n$} & $\bar{\nu}_{\mu} p$ & $\bar{\nu}_{\mu} n$ \\
\hline$\alpha_{\mathrm{ch}}$ & 0.40 & -0.20 & 0.02 & 0.80 \\
$\beta_{\mathrm{ch}}$ & 1.42 & 1.42 & 1.28 & 0.95 \\
$\mathrm{c}$ & 7.93 & 5.22 & 5.22 & 7.93 \\
\hline \hline
\end{tabular}

with the transverse mass of the hadron defined as $m_{\perp}^{2} \equiv$ $m^{2}+p_{\perp}^{2} / c$ and $z$ being quantities that characterize the hadronic shower [49]. The transverse momentum is defined as $p_{\perp}^{2}=p_{x}^{2}+p_{y}^{2} . z$ describes the fraction of available lightcone momentum $E+p_{z}$ transferred to the hadrons produced with energy $E$, and it is defined as $z=E / \nu$. The parameters $a$ and $b$, known as Lund $a$ and Lund $b$, are free parameters of the model that are responsible for distributing the longitudinal energy of the hadronic system after the interaction, and they should be tuned to reproduce experimental data [49]. In terms on the effect on $\left\langle n_{\mathrm{ch}}\right\rangle$, as Lund $a$ increases, the multiplicity increases as well, while the opposite is happening for Lund $b$.

In GENIE, PYTHIA is used to simulate the hadronization at high-energy invariant masses. Specifically, GENIE v3.00.06 uses PYTHIA6. Future GENIE releases will slowly transition to PYTHIA8. In particular, in v3.00.06, PYTHIA8 is partially integrated in GENIE and it is fully integrated in the AGKY model. After the partial integration of PYTHIA8, simulation outputs remained unchanged. Hence, the tune presented in this paper is also valid for PYTHIA8. Moreover, different GENIE comprehensive model configurations (CMCs) [24] have no impact on the hadronization predictions.

The default PYTHIA parameters shown in Table II come from fits to high-energy $e^{+}-e^{-}$experiments [50-61] $(\sqrt{s} \sim 35 \mathrm{GeV})$. PYTHIA's description of data at low energy, such as modern neutrino oscillation experiments $(1-10 \mathrm{GeV})$ or even lower-energy $e^{ \pm}-p$ experiments such as the HERMES experiment (at $27 \mathrm{GeV}$ ) [62], is not accurate; see Sec. IV. The first attempt to improve this disagreement was in 2010, when some of the PYTHIA parameters were tweaked according to a NUX PYTHIA tune [40]. The parameters modified by the NUX PYTHIA tune are as follows:

(i) $P_{s \bar{s}}$ controls the $s \bar{s}$ production suppression,

(ii) $\left\langle p_{\perp}^{2}\right\rangle$ determines the average hadron transverse momentum squared,

(iii) $E_{\text {CutOff }}$ is the energy cutoff for the fragmentation process.

These parameters are related to important hadron shower characteristics. The assumption of tunneling breakups implies the suppression of heavy-quark production, limiting its production in soft fragmentation processes. The

TABLE II. Summary of different PYTHIA parameterizations. The parameter configuration for PYTHIA, NUX, HERMES, and 2010 GENIE tunes are specified. The details on the HERMES tune are given in Sec. IV B.

\begin{tabular}{|c|c|c|c|c|c|}
\hline Parameter & Name in PYTHIA & PYTHIA default & NUX tune & HERMES tune & 2010 GENIE tune \\
\hline$\overline{P_{s \bar{s}}}$ & $\operatorname{PARJ}(2)$ & 0.30 & 0.21 & 0.25 & 0.30 \\
\hline$\left\langle p_{\perp}^{2}\right\rangle\left(\mathrm{GeV}^{2} / c^{2}\right)$ & PARJ(21) & 0.36 & 0.44 & 0.42 & 0.44 \\
\hline$E_{\text {CutOff }}(\mathrm{GeV})$ & PARJ(33) & 0.80 & 0.20 & 0.47 & 0.20 \\
\hline Lund $a$ & PARJ(41) & 0.30 & 0.30 & 0.68 & 0.30 \\
\hline Lund $b\left(c^{4} / \mathrm{GeV}^{2}\right)$ & PARJ(42) & 0.58 & 0.58 & 0.35 & 0.58 \\
\hline
\end{tabular}




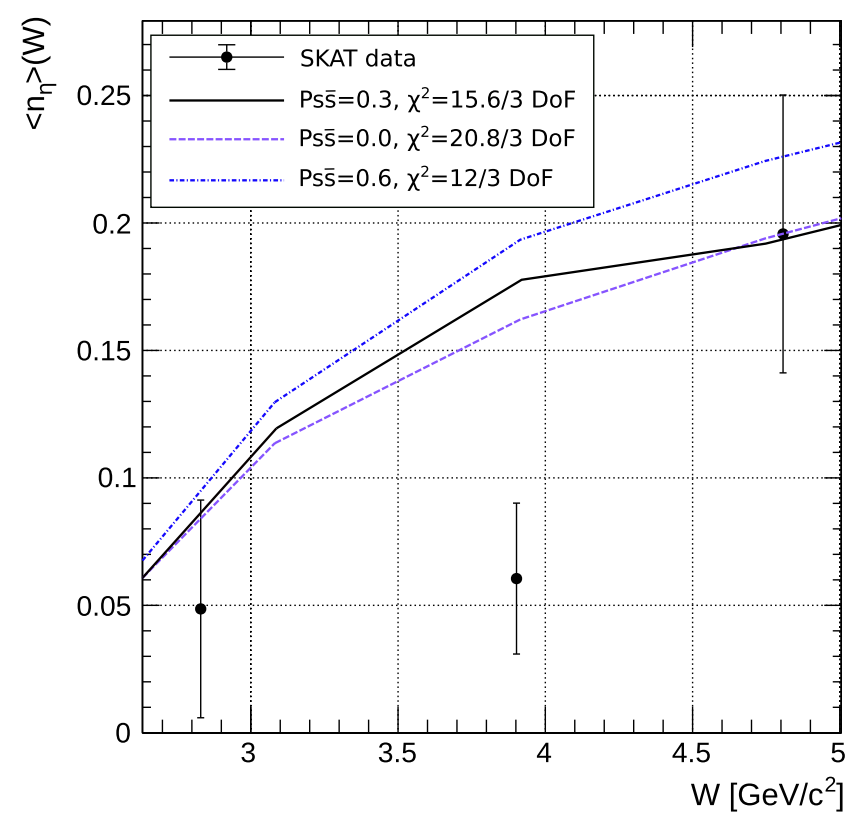

FIG. 4. Parameter impact on the averaged $\eta$ production data from SKAT [63].

suppression factor for heavy quarks is $u \bar{u}: d \bar{d}: s \bar{s}: c \bar{c} \sim$ $1: 1: 0.3: 10^{-11}$ [49]. This is supported by $\eta$ production data, Fig. 4. Previous tunes are in agreement with this fact; see Table II. Each quark-antiquark pair receives opposite $p_{\perp}$ kicks at each string breaking point according to a Gaussian distribution. The $\left\langle p_{\perp}^{2}\right\rangle$ parameter controls the variance of the Gaussian distribution used at the breaking point. There are different datasets available to constrain this parameter [27]; see, for instance, Fig. 5. Finally, $E_{\text {Cutoff }}$ determines the minimum energy at which the fragmentation of the parton system can occur, set to $0.8 \mathrm{GeV}$ in PYTHIA. 2010 GENIE uses the best-fit value from the NUX PYTHIA tune, where $E_{\text {Cutoff }}=0.20 \mathrm{GeV}$.

In GENIE v3 and previous releases, there is only one parameter set configuration for the low- $W$ AGKY model (Table I) and PYTHIA (Table II) that is common for all CMCs.

\section{NEUTRINO-INDUCED HADRONIZATION DATA REVIEW}

The characterization of the AGKY parameters relies on neutrino-induced hadronization data from BEBC and FNAL $15 \mathrm{ft}$ experiments. These experiments published a variety of observables related to hadronization. This work is based mainly on charged multiplicity data as a function of the hadronic invariant mass; hence, it is what this review is focusing on. The analyses procedure for both experiments are similar and depend on the target type that can be hydrogen or deuterium. The different analysis requirements need to be implemented in the GENIE hadronization analyses for a meaningful data/MC comparison; see Sec. VI. In this section, the analyses of interest for this work are discussed in detail.

\section{A. Hydrogen data}

The bubble chamber at Fermilab (FNAL $15 \mathrm{ft}$ ) and BEBC at CERN follow similar analyses procedures. The data considered in this work are those listed in Table III.

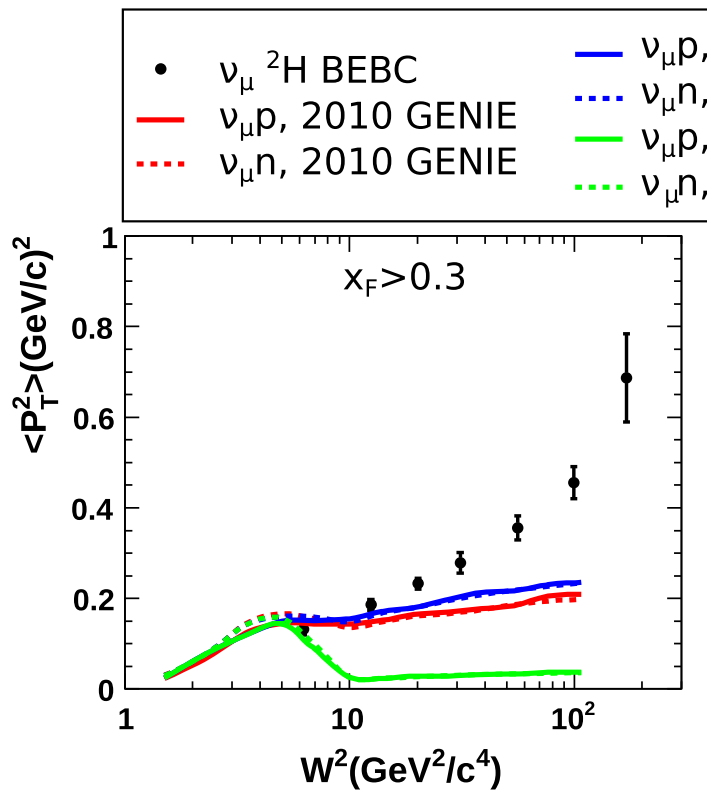


TABLE III. Compilation of historical data from the BEBC and FNAL $15 \mathrm{ft}$ bubble chamber experiments on averaged charged hadron multiplicity in muon (anti)neutrino on hydrogen interactions. Information about the number of points in each dataset, $N_{p}$; the $W^{2}$ range covered; and the cuts applied in each analysis is provided. Unless specified, the systematic errors were not included in the data release error bands and have been added in quadrature by the amount specified in this table; see details in Sec. III C. The sixth column specifies whether a dataset is included, discarded or partially included in the fit; see Sec. IV C. The complete list of data points removed in this analysis is specified in the Sec. VII.

\begin{tabular}{|c|c|c|c|c|c|c|}
\hline Experiment & $\mathbf{N}_{\mathbf{p}}$ & $\mathbf{W}^{2}\left(\mathrm{GeV}^{2} / \mathrm{c}^{4}\right)$ & Cuts & Systematic errors & In fit & References \\
\hline \multicolumn{7}{|c|}{$\nu_{\mu}+p \rightarrow \mu^{-}+X^{++}$} \\
\hline \multirow[b]{2}{*}{ FNAL 15 ft (1976) } & & & $E_{\nu}^{\text {reco }} \geq 15 \mathrm{GeV}$ & & & \\
\hline & 25 & {$[1.5,150]$} & $\begin{array}{c}p_{L}^{\text {visible }} \geq 10 \mathrm{GeV} / c \\
p^{\mu} \geq 5 \mathrm{GeV} / c \\
p_{T}^{\mu} \geq 1 \mathrm{GeV} / c\end{array}$ & Included & $W^{2}<20 \mathrm{GeV}^{2} / c^{4}$ & [64] \\
\hline \multirow[b]{2}{*}{ BEBC (1983) } & & & $p^{\mu} \geq 3 \mathrm{GeV} / c$ & & & \\
\hline & 11 & {$[9,121]$} & $\begin{array}{c}E^{\text {visible }} \geq 5 \mathrm{GeV} \\
W^{2} \geq 9 \mathrm{GeV}^{2} / \mathrm{c}^{4}\end{array}$ & $3-5 \%$ & $x$ & [65] \\
\hline \multirow[b]{2}{*}{ BEBC (1990) } & & & $Q^{2} \geq 1(\mathrm{GeV} / c)^{2}$ & & & \\
\hline & 6 & {$[6,150]$} & $\begin{array}{c}p^{\mu} \geq 3 \mathrm{GeV} / c \\
W^{2} \geq 4 \mathrm{GeV}^{2} / c^{4}\end{array}$ & Statistical & $W^{2}<9 \mathrm{GeV}^{2} / c^{4}$ & [66] \\
\hline BEBC (1992) & 5 & {$[12,144]$} & $p^{\mu} \geq 3 \mathrm{GeV} / c$ & Included & $\checkmark$ & [67] \\
\hline \multicolumn{7}{|c|}{$\bar{\nu}_{\mu}+p \rightarrow \mu^{+}+X^{0}$} \\
\hline \multirow[b]{2}{*}{ FNAL 15 ft (1981) } & & & $\begin{array}{l}p_{\mathrm{ch}} \geq 5 \mathrm{GeV} / c \\
p_{\mathrm{FW}}^{\text {tot }} \geq 2 \mathrm{GeV} / c\end{array}$ & & & \\
\hline & 10 & {$[16,100]$} & $\begin{array}{c}y_{\mathrm{B}} \geq 0.1 \\
y_{\mathrm{B}} \leq 0.8 \\
E_{\bar{\nu}}^{\mathrm{reco}} \geq 5 \mathrm{GeV}\end{array}$ & Statistical & $W^{2}<30 \mathrm{GeV}^{2} / c^{4}$ & [68] \\
\hline BEBC (1983) & 10 & {$[9,121]$} & $\begin{array}{c}p^{\mu} \geq 3 \mathrm{GeV} / c \\
E^{\text {visible }} \geq 5 \mathrm{GeV} \\
W^{2} \geq 9 \mathrm{GeV}^{2} / c^{4}\end{array}$ & $3-5 \%$ & $x$ & [65] \\
\hline & & & $Q^{2} \geq 0.1(\mathrm{GeV} / c)^{2}$ & & & \\
\hline BEBC (1990) & 6 & {$[6,144]$} & $\begin{array}{c}p^{\mu} \geq 3 \mathrm{GeV} / c \\
W^{2} \geq 4 \mathrm{GeV}^{2} / c^{4}\end{array}$ & Statistical & $W^{2}<10 \mathrm{GeV}^{2} / c^{4}$ & [66] \\
\hline BEBC (1992) & 5 & {$[12,144]$} & $p^{\mu} \geq 3 \mathrm{GeV} / c$ & Included & $W^{2}<60 \mathrm{GeV}^{2} / c^{4}$ & [67] \\
\hline
\end{tabular}

Both experiments look for $\nu_{\mu}$ and $\bar{\nu}_{\mu} \mathrm{CC}$ interactions on hydrogen to study the averaged charged multiplicity of the final state as a function of the event invariant mass. The main requirement to select $\mathrm{CC}$ events is to detect a muon track. Muons are detected with an external muon identifier (EMI), and a minimum muon momentum, $p_{\mu}$, is usually required to guarantee good muon identification (ID). This is a consequence of the muon ID efficiency dependence on the muon momentum energy. For instance, in BEBC experiment, the muon ID efficiency varies from $40 \%$ to $100 \%$ in the muon momentum range of $3 \mathrm{GeV} / c \leq$ $p_{\mu} \leq 10 \mathrm{GeV} / c$, with an average efficiency of $95 \%$. The FNAL $15 \mathrm{ft}$ experiment also uses a kinematic technique to identify negative muons in neutrino interactions [69]. Under this $\mu^{-}$-ID method, only events in which the $\mu^{-}$candidate has transverse momentum, $p_{\perp}^{\mu}$, of at least $1 \mathrm{GeV} / c$ are accepted.

Selected events, which satisfy the conditions specified above, are analyzed to reconstruct the event topology and kinematics. In particular, BEBC uses the HYDRA program [65-67] and FNAL $15 \mathrm{ft}$ a modified version of the TVGP program [70]. Only a small fraction of the charged finalstate hadrons is identified by using energy loss, range in hydrogen, break point probability, and kinematic fits [65]. If left unidentified, the remaining charged hadrons are assumed to be pions; this assumption can cause migration 
of particles from the backward- to the forward-going hemispheres. For instance, the BEBC experiment is able to identify about $30 \%$ of the protons using the HYDRA algorithm, while the rest are classified as pions [66].

For $\nu_{\mu} \mathrm{CC}$ interactions, because of charge conservation, the experiments scan for events with three or more charged particles in the final state.

The topology of neutrino and antineutrino events is expected to be different. In antineutrino events, interactions with only one charged track can occur $\left(n_{\mathrm{ch}}=0\right)$. Such events are not negligible at low $E_{\nu}$ and low $W$. However, these are removed due to low scanning efficiency and poor antineutrino energy reconstruction. Both BEBC and FNAL $15 \mathrm{ft}$ correct for the effect of removing one-prong contributions in antineutrino samples using $\mathrm{MC}$ calculations [65-68]. One-prong MC events are weighted so that the fraction of one-prong events agrees with the experimental estimate. The scanning efficiencies for three-prong events are higher than $90 \%$, improving as the number of charged secondaries increases $(\geq 95 \%)$.

In hydrogen and deuterium bubble chambers, the identification of neutral particles, such as $\pi^{0}$, is difficult due to the low $Z$ of the medium. As a consequence, the transverse momentum balance method is used to estimate the neutrino energy by assuming undetected neutral particles in the event [71],

$$
E_{\nu}^{\mathrm{reco}}=p_{L}^{\mu} c+p_{L}^{\mathrm{ch}} c\left(1+\frac{\left|\boldsymbol{p}_{\perp}^{\mu}+\boldsymbol{p}_{T}^{\mathrm{ch}}\right|}{\sum_{i=1}^{n_{\mathrm{ch}}}\left|\boldsymbol{p}_{\perp i}\right|}\right) .
$$

The subscripts $L$ and $\perp$ refer to longitudinal and transverse components of the momenta relative to the neutrino direction, whereas the $c h$ and $\mu$ labels denote the charged-hadron system and the muon, respectively. The index $i$ runs over the charged hadrons in the hadronic system. By using this method, there is a non-negligible bias for the neutrino energy reconstruction. For instance, the BEBC experiment estimated the reconstructed neutrino energy to differ from the true energy by $\sim 10-15 \%[38,65]$. Both bubble chambers corrected for this effect; see Sec. III C. In some analyses, cuts on the reconstructed neutrino energy, $E_{\nu}^{\text {reco }}$, are applied $[38,64,65]$.

Backgrounds from NC events, quasielastic (QEL) CC events, or neutral-particle-induced events are removed from the final sample using kinematic cuts that depend on each analysis. $\mathrm{NC}$ events can mimic CC events as a consequence of muon-hadron misidentification. On the one hand, for the FNAL $15 \mathrm{ft}$ experiment, the muon-hadron misidentification increases at high Bjorken inelasticity values $\left(y_{\mathrm{B}}\right)$, and a cut on $y_{\mathrm{B}}$ is required to guarantee a good efficiency in selecting CC events [68]. In $\bar{\nu}_{\mu}$ events, backgrounds from low-energy neutrons as well as events caused by incoming hadron tracks that rescatter within the chamber are controlled by requiring the total momentum in the forward hemisphere, $p_{\mathrm{FW}}^{\text {tot }}$, to be greater than $2 \mathrm{GeV} / c[68,70]$. Moreover, FNAL $15 \mathrm{ft}$ removes backgrounds from $K_{L}^{0}$ mesons by requiring the minimum total momentum from charged particles, $p_{\mathrm{ch}}$, to be higher than $5 \mathrm{GeV} / c$ [68]. On the other hand, the BEBC experiment applies kinematic cuts on either $W$ or/ and $Q^{2}$ to remove QEL events [65-67]. All cuts applied to the different analyses are shown in Table III.

\section{B. Deuterium data}

The analyses algorithm followed by the FNAL $15 \mathrm{ft}$ and BEBC bubble chamber experiments operating with deuterium aims to discriminate between interactions on proton and neutron. The data on deuterium considered in this work are those listed in Table IV.

Before classifying the event as a neutrino interaction on either proton or neutron, the analyses procedure is equivalent to the one described in Sec. III. Each event has to contain a muon, identified with the EMI, that satisfies the cuts summarised in Table IV. The information about the event topology and kinematics is obtained using the TVGP-SQUAW or HYDRA algorithms for FNAL $15 \mathrm{ft}$ [38] and $\mathrm{BEBC}$, respectively $[39,72,73]$. Particles are classified as pions if the algorithm fails to identify them as any other particle. The neutrino energy is reconstructed using the transverse momentum balance method. Similar kinematic cuts to those specified for the hydrogen analyses are applied.

The main difference between both analyses is the particle identification of struck nucleons in the event. A neutrino event is classified as a neutrino interaction on proton if the event topology has an odd number of prongs. Alternatively, the event is classified as an interaction on neutron if the event has an even number of prongs with no visible spectator or an odd number of prongs that include a visible proton. See a graphical interpretation in Fig. 6. The antineutrino case is similar except that the minimum prong multiplicity on proton is 1 , instead of 3 . Because of the selection criteria explained in Sec. III, interactions with $n_{\mathrm{ch}}=0$ are not considered, effectively making the selection criteria for antineutrinos the same as for neutrinos.

In the analyses, a prong is classified as a proton if it corresponds to a particle moving backward relatively to the beam direction $\left(\cos \theta_{p}<0\right)$ or a forward-going particle with low momentum. The maximum momentum cut is dataset dependent; see Table IV. If these conditions are not satisfied, the proton is not reconstructed, and for the purpose of the analyses, it is considered invisible. In the FNAL $15 \mathrm{ft}$ analyses, for a proton to be detected as a prong, its momentum has to be $p_{p}>200 \mathrm{MeV} / c$.

The deuterium target can induce rescattering of the hit nucleon with the spectator; this can increase the number of hadrons in the final state [69]. An odd number of prongs can occur in any possible neutrino interaction because of rescattering, independently of the hit nucleons, so the $\nu_{\mu} p$ sample will contain $\nu_{\mu} n$ events. In contrast, the $\nu_{\mu} n$ sample can only contain $\nu_{\mu} p$ events because of detector inefficiencies. Rescattering events have an impact on the event kinematics, which can be quantified defining an energy balance as 
TABLE IV. Compilation of historical data from the BEBC and FNAL $15 \mathrm{ft}$ bubble chamber experiments on averaged charged hadron multiplicity in muon (anti)neutrino on deuterium interactions. Information about the number of points in each dataset, $N_{p}$; the $W^{2}$ range covered; and the cuts applied in each analysis is provided. Unless specified, the systematic errors were not included in the data release error bands and have been added in quadrature by the amount specified in this table; see details in Sec. III C. The sixth column specifies whether a dataset is included, discarded, or partially included in the fit; see Sec. IV C.

\begin{tabular}{|c|c|c|c|c|c|c|}
\hline Experiment & $\mathbf{N}_{\mathrm{p}}$ & $\mathbf{W}^{2}\left(\mathrm{GeV}^{2} / c^{4}\right)$ & Cuts & Systematic errors & In fit & References \\
\hline \multicolumn{7}{|c|}{$\nu_{\mu}+p \rightarrow \mu^{-} X^{++}$} \\
\hline & & & $\begin{array}{l}p_{\mu} \geq 5 \mathrm{GeV} / c \\
p_{\mu}^{\perp} \geq 1 \mathrm{GeV} / c\end{array}$ & & & \\
\hline FNAL 15 ft (1983) & 14 & {$[1,225]$} & $\begin{array}{c}p_{\mathrm{ch}}^{L} \geq 5 \mathrm{GeV} / c \\
p_{p} \leq 340 \mathrm{MeV} / c \\
p_{p} \geq 200 \mathrm{MeV} / c \\
W \geq 1.5 \mathrm{GeV} / c^{2} \\
E_{\nu}^{\text {reco }} \geq 10 \mathrm{GeV}\end{array}$ & $10 \%$ & $W^{2}>4 \mathrm{GeV}^{2} / c^{4} \dagger$ & [38] \\
\hline BEBC (1989) & 6 & {$[4,196]$} & $\begin{array}{c}\varepsilon_{\text {cut }} \\
p^{\mu} \geq 4 \mathrm{GeV} / c \\
p_{p} \leq 300 \mathrm{MeV} / c\end{array}$ & Not included & $x$ & [72] \\
\hline \multicolumn{7}{|c|}{$\nu_{\mu}+n \rightarrow \mu^{-} X^{+}$} \\
\hline FNAL 15 ft (1983) & 14 & {$[1,225]$} & $\begin{array}{c}p_{\mu}^{\perp} \geq 1 \mathrm{GeV} / c \\
p_{L}^{\text {ch }} \geq 5 \mathrm{GeV} / c \\
E_{\nu}^{\text {reco }} \geq 10 \mathrm{GeV} \\
p_{p} \leq 340 \mathrm{MeV} / c \\
p_{p} \geq 200 \mathrm{MeV} / c\end{array}$ & $10 \%$ & $\checkmark$ & [38] \\
\hline BEBC (1984) & 8 & {$[6,112]$} & $\begin{array}{c}\varepsilon_{\text {cut }} \\
p_{\mu} \geq 4 \mathrm{GeV} / c \\
Q^{2} \geq 1(\mathrm{GeV} / c)^{2} \\
W^{2} \geq 5 \mathrm{GeV}^{2} / c^{4} \\
p_{p} \leq 300 \mathrm{MeV} / c\end{array}$ & Statistical & $\checkmark$ & [73] \\
\hline BEBC (1989) & 6 & {$[4,196]$} & $\begin{array}{c}\varepsilon_{\text {cut }} \\
p_{\mu} \geq 4 \mathrm{GeV} / c \\
p_{p} \leq 300 \mathrm{MeV} / c \\
W \geq 5 \mathrm{GeV} / c^{2}\end{array}$ & Not included & $x$ & [72] \\
\hline \multicolumn{7}{|c|}{$\bar{\nu}_{\mu}+p \rightarrow \mu^{+} X^{0}$} \\
\hline BEBC (1982) & 8 & {$[5,75]$} & $\begin{array}{c}p_{\mu} \geq 4 \mathrm{GeV} / c \\
p_{p} \leq 300 \mathrm{MeV} / c\end{array}$ & Statistical & $\checkmark$ & [39] \\
\hline BEBC (1989) & 6 & {$[4,196]$} & $\begin{array}{c}\varepsilon_{\text {cut }} \\
p^{\mu} \geq 4 \mathrm{GeV} / c \\
p_{p} \leq 300 \mathrm{MeV} / c \\
\end{array}$ & Not included & $x$ & [72] \\
\hline \multicolumn{7}{|c|}{$\bar{\nu}_{\mu}+n \rightarrow \mu^{+} X^{-}$} \\
\hline BEBC (1982) & 8 & {$[1.5,56]$} & $\begin{array}{c}p_{\mu} \geq 4 \\
p_{p} \leq 300 \mathrm{MeV} / c\end{array}$ & Statistical & $\checkmark$ & [39] \\
\hline BEBC (1989) & 6 & {$[4,196]$} & $\begin{array}{c}\varepsilon_{\text {cut }} \\
p_{\mu} \geq 4 \mathrm{GeV} / \mathrm{c} \\
p_{p} \leq 300 \mathrm{MeV} / c \\
\end{array}$ & Not included & $x$ & [72] \\
\hline
\end{tabular}




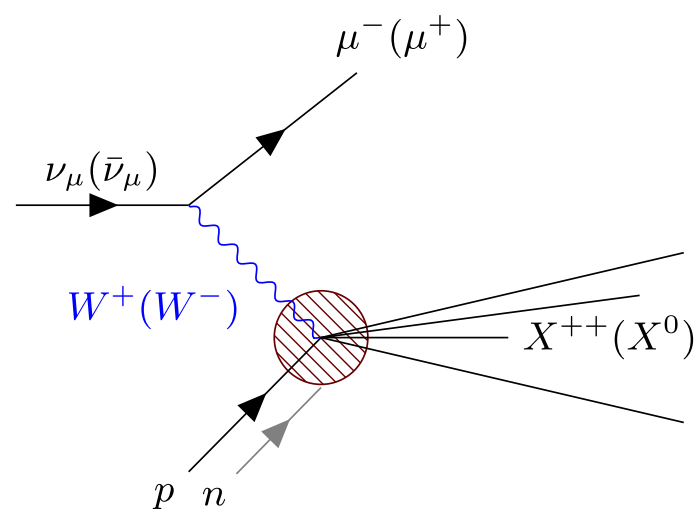

(a)

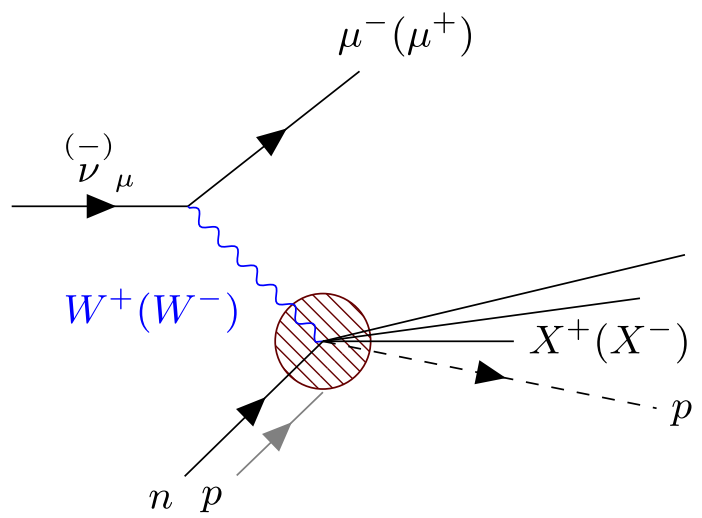

(b)

FIG. 6. Bubble chamber analyses of $\nu_{\mu}$ and $\bar{\nu}_{\mu}$ on ${ }^{2} \mathrm{H}$ data schematic procedure. The topology definition is based mainly on the number of prongs in each event. Possible visible proton spectators that satisfy the momentum requirements specified in Table IV are represented with dashed lines. $\bar{\nu}_{\mu}{ }^{2} \mathrm{H}$ one-prong events are not considered. (a) $\nu_{\mu} p$ topology and (b) $\nu_{\mu} n$ topology.

$$
\varepsilon \equiv \sum_{i}\left(E_{i}-p_{L i} c\right)-M c^{2}
$$

where $E_{i}$ and $p_{L i}$ are the $i$ th charged-particle energy and longitudinal momentum component relative to the neutrino direction, respectively, while $M$ is the mass of the target nucleon assumed in the selection sample. Equation (7) assumes that the nucleon is at rest and that the neutrino direction is known. In an ideal detector where all final-state particles are identified, $\varepsilon=0$ [74]. In a bubble chamber experiment, where only charged particles are detected, $\varepsilon<0$. Possible particle misidentification reduces the $\varepsilon$ value further, as particles are assigned to be pions as a default, unless identified otherwise. Rescattering events have a $\varepsilon>0$ with a maximum value of $M_{{ }_{2} \mathrm{H}} c^{2}-M_{n} c^{2}$; see Fig. 7 .

The BEBC experiment eliminates rescattering events from the sample by imposing a cut on the energy balance $[39,72,73]$. An event is rejected due to rescattering if the following conditions are satisfied:

$\varepsilon>0.1 \mathrm{GeV}$,

$\varepsilon>-0.1 \mathrm{GeV}$ and the transverse missing momenta squared differs from zero, $\left(p_{\perp}^{\text {miss }}\right)^{2}>0.075(\mathrm{GeV} / c)^{2}$. The FNAL $15 \mathrm{ft}$ experiment did not correct for rescattering events. In some of the analyses, additional cuts are considered for the deuterium analyses to remove backgrounds. For instance, the FNAL $15 \mathrm{ft}$ bubble chamber reduces the background from neutral hadroninduced events by applying a cut on the total chargedparticle longitudinal momentum in the final-state system $\left(p_{\mathrm{ch}}^{L}\right)$ [38].

\section{Sources of systematic uncertainties in the FNAL $15 \mathrm{ft}$ and BEBC experiments}

MC studies were preformed by the FNAL $15 \mathrm{ft}$ and BEBC bubble chamber experiments to correct for possible

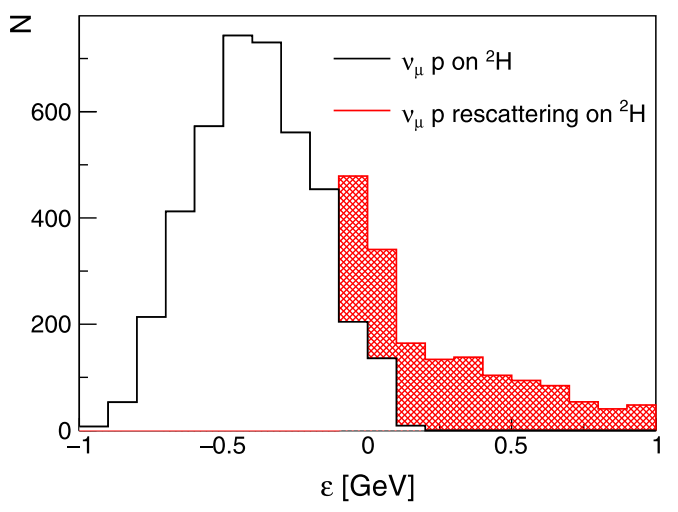

(a)

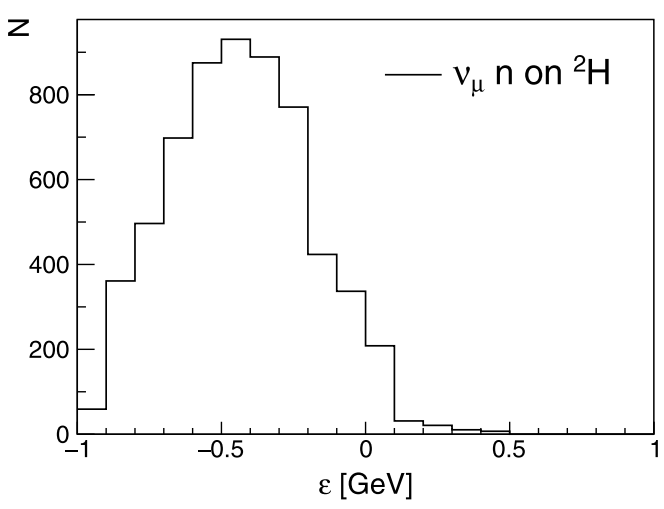

(b)

FIG. 7. Energy balance distribution for $\nu_{\mu}$ events on proton and neutron candidates digitized from the BEBC analyses paper [73]. Events that do not satisfy the $\epsilon_{\text {reco }}$ correspond to rescattering events and are highlighted in red. No rescattering contribution were observed in the $\nu_{\mu} n$ sample. (a) $\nu_{\mu} p$ events under the odd prong topology assumption and (b) $\nu_{\mu} n$ events under the even prong topology assumption. Neutron events with a spectator proton are not included. 
sources of errors. In particular, the different analyses correct for the following effects:

EMI geometrical inefficiency $[39,66,67,73]$.

Efficiency losses due to possible hadron misidentification and migration of particles from the forward to backward hemispheres [65-67,73].

$W^{2}$ smearing due to the uncertainty in the neutrino energy reconstruction [38,39,64-68,72,73].

Neutrino energy uncertainty associated the transverse balance method [38,39,64-68,72,73].

Neutral particle decays $\left(\gamma, K^{0}\right.$, and $\left.\Delta\right)$ into charged particles that can lead to a higher charged multiplicity if the decay vertex is close to the primary one [38,39,64-68].

One-prong event corrections [65-68,73]. This kind of events occurs for low- $W \bar{\nu} p$ interactions in which only the $\mu^{+}$is observed.

Efficiency to detect CC events $[38,68]$.

Corrections due to the Fermi motion in deuterium [73].

Possible measurement errors $[38,65-67,73]$.

The information about the BEBC systematic errors was obtained by using two MC programs: the LUND MC and a longitudinal phase space model [75]. Both MC were tuned to describe the BEBC experiment. From the MC generations, two samples are created: the initial, $d_{\text {initial }}^{\mathrm{MC}}$, and modified, $d_{\text {modified }}^{\mathrm{MC}}$, samples. The initial sample contains the truth information of the event. The modified sample includes modifications to mimic the analyses procedure. The ratio between the samples provides a correction factor that it is applied to the data.

BEBC systematic errors are obtained from the difference between both MC calculations. The FNAL $15 \mathrm{ft}$ corrected for some of the effects, but no clear information about the methodology followed to estimate the systematics is provided. Some of these experiments provide error bars which already include an estimation of systematics; however, this is not the case for most of the data. In particular, there are three different ways in which the BEBC and FNAL $15 \mathrm{ft}$ experiments quote the systematic errors: (1) The systematic errors are already included in the total error, (2) The systematic uncertainty was quoted as a percentage with respect to the central value. (3) The systematic error is considered to be approximately of the same size of the statistical error. (4) No information is provided in the data release. For cases 2 and 3, the systematic errors are added in quadrature to the statistical ones in this analysis. Particularly, for the datasets from Ref. [72], information on systematic errors is not provided in the data release. In Tables. III and IV, the information on the systematic error is provided. We label the different categories as (1) included, (2) with the percentage, (3) statistical, and (4) not included, respectively. No correlation matrices are provided by any of these experiments.

\section{REVIEW OF PREVIOUS TUNES TO HADRONIZATION DATA}

While summarizing the experimental fits to averaged charged multiplicity data, this section also explains the origin of the 2010 GENIE tune parameters. This is necessary to define proper selection criteria for a dataset to be included in a global fit.

\section{A. Fits to bubble chamber data}

Both BEBC and FNAL $15 \mathrm{ft}$ experiments provided estimations of the $\alpha_{\mathrm{ch}}$ and $\beta_{\mathrm{ch}}$ parameters for every released dataset. The individual fits were performed by fitting Eq. (1) in each channel. Fit results are summarized in Table V.

There are six channels in total, $\nu_{\mu}$ or $\bar{\nu}_{\mu}$ on the proton or neutron, while the information on interactions on proton can be from data with hydrogen or deuterium targets. Information about neutrino interaction on the neutron can only be extracted from deuterium samples. The BEBC and/ or FNAL $15 \mathrm{ft}$ experiments performed individual fits to each of the available channels.

From the best-fit values extracted for each dataset, we observe clear discrepancies for the $\alpha_{\mathrm{ch}}$ and $\beta_{\mathrm{ch}}$ values between data releases and between the BEBC and FNAL $15 \mathrm{ft}$ data (e.g., for $\nu_{\mu} p$ interactions on hydrogen). Discrepancies between hydrogen and deuterium samples are also present. This target-related discrepancy can also be observed in fits to OPERA and CHORUS data $[43,76]$. These discrepancies could have different origins: the $W^{2}$ range, the beam energy, or the kinematic cuts applied in the analyses.

The 2010 GENIE AGKY parameter values presented in Table I correspond to the analyses on deuterium targets highlighted in Table V. Notice that the parameters used in the 2010 GENIE prediction come from fits to Eq. (1) over the whole $W^{2}$ range. This procedure is not adequate as the $\alpha_{\mathrm{ch}}$ and $\beta_{\text {ch }}$ should be extracted from a fit to data over the low$W$ validity range given that the AGKY model differs from the simplified linear behavior.

The description of the shower particle content is linked to several observables whose correlation is still unknown. For instance, the averaged charged multiplicity and dispersion observables can be correlated. The full list of available hadronization data is shown in Ref. [27]. Ideally, the AGKY tune should improve the agreement with all hadronization related observables. The extraction of the averaged charged multiplicity parameters, such as $\alpha_{\mathrm{ch}}$ and $\beta_{\mathrm{ch}}$, strongly relays on the precise understanding of the datasets described in Secs. III and III B. However, the analyses of historical averaged charged multiplicity datasets already show clear disagreements between each of the different data releases, as summarized in Table V. For these reasons, on this work, we focus on the description and tune of averaged charged multiplicity data on hydrogen and deuterium samples. 
TABLE V. Compilation of best-fit values for the intercept $\alpha_{\mathrm{ch}}$ and slope $\beta_{\mathrm{ch}}$ obtained from individual fits to Eq. (1) against mean charged hadron multiplicity data as a function of $W^{2}$. The parameters for charged-current $\nu_{\mu}$ and $\bar{\nu}_{\mu}$ scattering data on hydrogen, deuterium, ${ }^{207} \mathrm{~Pb}$, and the Fuji ET-B7 emulsion are shown in the table. 2010 GENIE parameters are extracted from the analyses highlighted in bold.

\begin{tabular}{|c|c|c|c|c|c|}
\hline Experiment & $\mathbf{W}^{2}\left(\mathrm{GeV}^{2} / c^{4}\right)$ & Target & $\alpha_{\mathrm{ch}}$ & $\beta_{\mathrm{ch}}$ & References \\
\hline \multicolumn{6}{|c|}{$\nu_{\mu}+p \rightarrow \mu^{-} X^{++}$} \\
\hline FNAL 15 ft (1976) & {$[1.5,150]$} & $\mathrm{H}$ & $1.09 \pm 0.38$ & $1.09 \pm 0.03$ & [64] \\
\hline BEBC (1983) & {$[12,112]$} & $\mathrm{H}$ & $-0.05 \pm 0.11$ & $1.43 \pm 0.04$ & [65] \\
\hline FNAL $15 \mathrm{ft}(1983)$ & {$[1.5,160]$} & ${ }^{2} \mathrm{H}$ & $0.05 \pm 0.07$ & $1.42 \pm 0.03$ & [38] \\
\hline BEBC (1990) & {$[6,150]$} & $\mathrm{H}$ & $0.911 \pm 0.224$ & $1.131 \pm 0.086$ & [66] \\
\hline BEBC (1992) & {$[12,144]$} & $\mathrm{H}$ & $0.40 \pm 0.13$ & $1.25 \pm 0.04$ & [67] \\
\hline \multicolumn{6}{|c|}{$\nu_{\mu}+n \rightarrow \mu^{-} X^{+}$} \\
\hline BEBC (1984) & {$[6,112]$} & ${ }^{2} \mathrm{H}$ & $1.75 \pm 0.12$ & $1.31 \pm 0.04$ & [73] \\
\hline FNAL $15 \mathrm{ft}$ (1983) & {$[1.5,160]$} & ${ }^{2} \mathrm{H}$ & $-0.20 \pm 0.07$ & $1.42 \pm 0.03$ & [38] \\
\hline \multicolumn{6}{|c|}{$\bar{\nu}_{\mu}+p \rightarrow \mu^{+} X^{0}$} \\
\hline FNAL 15 ft (1982) & {$[1.7,74]$} & $\mathrm{H}$ & $-0.44 \pm 0.13$ & $1.48 \pm 0.06$ & [68] \\
\hline BEBC (1982) & {$[5,75]$} & ${ }^{2} \mathrm{H}$ & $0.02 \pm 0.20$ & $1.28 \pm 0.08$ & [39] \\
\hline BEBC (1983) & {$[12,96]$} & $\mathrm{H}$ & $-0.56 \pm 0.25$ & $1.42 \pm 0.08$ & [65] \\
\hline BEBC (1990) & {$[6,144]$} & $\mathrm{H}$ & $0.222 \pm 0.362$ & $1.117 \pm 0.100$ & [66] \\
\hline BEBC (1992) & {$[12,144]$} & $\mathrm{H}$ & $-0.44 \pm 0.20$ & $1.30 \pm 0.06$ & [67] \\
\hline \multicolumn{6}{|c|}{$\bar{\nu}_{\mu}+n \rightarrow \mu^{+} X^{-}$} \\
\hline BEBC (1982) & {$[1.5,56]$} & ${ }^{2} \mathrm{H}$ & $0.80 \pm 0.09$ & $0.95 \pm 0.04$ & [39] \\
\hline \multicolumn{6}{|c|}{$\nu_{\mu}+A$} \\
\hline OPERA (2018) & {$[1.6,54.6]$} & $\mathrm{Pb}$ & $-0.19 \pm 0.18$ & $0.76 \pm 0.07$ & [43] \\
\hline CHORUS (2007) & {$[1,148]$} & Fuji ET-B7 & $1.07 \pm 0.05$ & $1.32 \pm 0.11$ & [76] \\
\hline
\end{tabular}

\section{B. HERMES tune}

The PYTHIA parameters are extracted from high-energy $e^{-} e^{+}$experiments; see Sec. II B. From Fig. 8, we see that PYTHIA underestimates the averaged charged multiplicity. However, PYTHIA has not been tuned using data from neutrino experiments.

The 2010 GENIE tune, summarized in Sec. II B, aimed to improve the agreement with different hadronization observables by incorporating the results from a NUX PYTHIA tune [40]. However, this was not sufficient to improve the agreement of PYTHIA with average charged multiplicity data from bubble chambers experiments. Moreover, the tune lacked information about the uncertainties of the fit parameters.

Information on PYTHIA parameters at lower energy was provided by the HERMES experiment, which tuned PYTHIA using $e^{ \pm} p$ data at $27 \mathrm{GeV}$ [62]. Reference [41] suggests that the HERMES tune improves the agreement with neutrino data, as summarized in Fig. 8. The main differences between the HERMES tune and the GENIE 2010 retune are the modification of the Lund $a$ and Lund $b$ parameters, suggesting higher (lower) values of Lund $a$ (Lund $b$ ).

The PYTHIA parameters with the most impact on the average charged multiplicity for the 2010 GENIE AGKY and HERMES tunes are summarized in Table II.

\section{Requirements for including a dataset in the AGKY multiplicity tune}

Only the averaged charged multiplicity data on hydrogen and deuterium is taken into account in this AGKY fit. Tables III-IV summarize the information about which datasets are included in the tune. If possible, only the latest analysis of each experiment is included. Previous analyses are only considered if:

(1) its reanalyses did not cover all the original $W^{2}$ range,

(2) the prediction interpolation by Professor fails to describe the GENIE prediction (see Sec. VI A),

(3) the data release lack of sufficient information about systematic errors.

In requirement 1 , previous analyses are used to complement the covered $W^{2}$ range, as those points were not documented in the revisited ones. If the datasets are only included partially, the approximate $W^{2}$ range used is provided. An example is the BEBC $\nu_{\mu}$ on $\mathrm{H}$ data, in particular datasets (BEBC,1) and (BEBC,2). In this case, the data point at $W^{2}<10 \mathrm{GeV}^{2} / c^{4}$ from the earlier release $(\mathrm{BEBC}, 1)$ is included in the fit, while the others are not because the later $(\mathrm{BEBC}, 2)$ is covering the same $W$ region. This approach has already been implemented in other studies [42]. The exact $W^{2}$ range after requirement 2 is given in Sec. VII. 


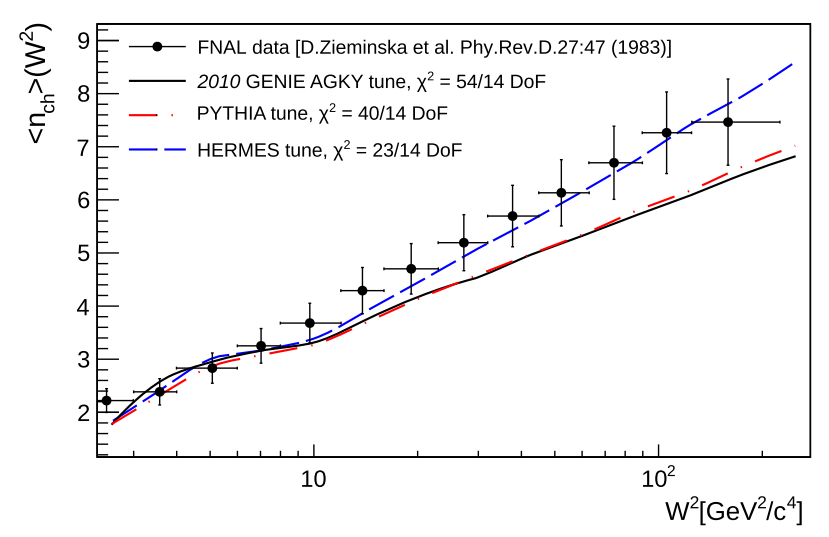

(a) $\nu_{\mu} p \rightarrow \mu^{-} X^{++}$on ${ }^{2} \mathrm{H}$.

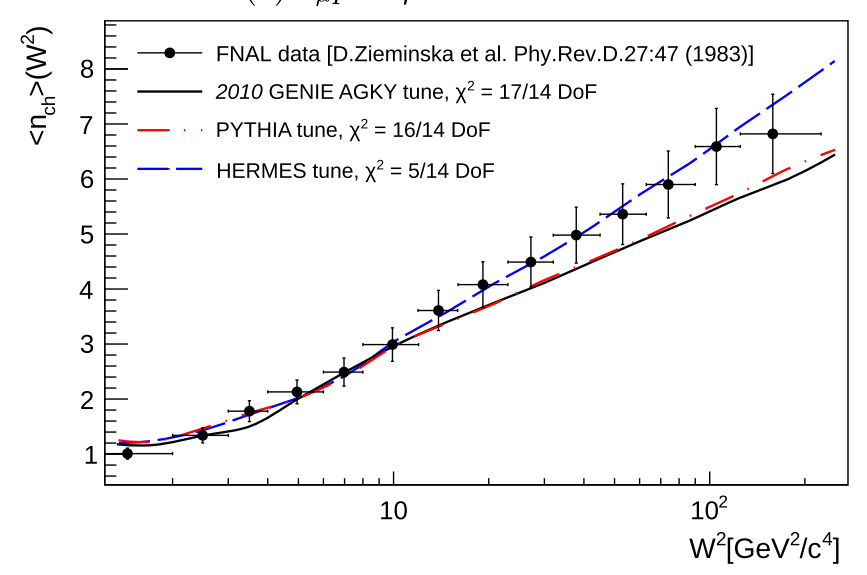

(b) $\nu_{\mu} n \rightarrow \mu^{-} X^{+}$on ${ }^{2} \mathrm{H}$.

FIG. 8. Comparison of FNAL average charged multiplicity deuterium data against GENIE predictions obtained with the parametrizations specified in Table II.

Global fits can be used to expose datasets that pull the results in different directions. This is the case of the most recent $\bar{\nu}_{\mu}$ measurement by BEBC experiment [72], which did not provide information on systematic errors, and consequently, the total error on this data tends to be much smaller than the rest; see Sec. VII. Such small errors give a strong preference to this dataset, and as a consequence, this measurement is in tension with other data, including older $\bar{\nu}_{\mu}$ BEBC measurements [39] for which information on the systematic uncertainty was provided; see Table IV. Given that the BEBC [72] analyses did not provide enough information on the systematic errors and they are in clear disagreement with the other ones, these are not considered in the tune and are shown for comparison only.

\section{PARAMETRIZATION OF MODEL UNCERTAINTIES}

This section discusses the impact on AGKY parameters on the predictions. The predictions are generated with the G18_02a_02_11a tune of GENIE v3.0.6. This tune was previously obtained to improve the agreement with pion production data on the free nucleon [24]. The complete
TABLE VI. Complete list of models used for the G18_02a_02_11a/b CMC in GENIE v3.

\begin{tabular}{lc}
\hline \hline Simulation domain & Model \\
\hline Nuclear model & Fermi gas [77] \\
QEL & Llewellyn Smith [79] \\
QEL charm & Kovalenko [80] \\
QEL $\Delta S=1$ & Pais [81] \\
RES & Rein-Sehgal [82] \\
SIS/DIS & Bodek-Yang [78] \\
DIS $\Delta S=1$ & Aivazis-Olness-Tung [83] \\
Coherent $\pi$ production & Rein-Sehgal [82] \\
Hadronization & AGKY [27] \\
FSI & INTRANUKE hA [84] \\
\hline \hline
\end{tabular}

model list for this tune is summarized in Table VI. As introduced in Sec. II, hadronization is modeled with the AGKY model [27]. Interactions with nuclei are calculated within the relativistic Fermi Gas framework, using the Bodek-Ritchie model [77], and hadronic reinteractions are simulated using INTRANUKE hA. The main contributions to the averaged charged multiplicity predictions come from CC DIS and nonresonance SIS [78]. As the DIS and models are common for all GENIE v3 tunes, the choice of the base configuration does not affect the hadronization predictions. An updated version of the G18_02a_02_11a tune, named G18_02a_02_11b, has been recently released in Ref. [24]. In terms of the hadronization predictions, these CMCs are interchangeable, and the results of this work are valid within the updated version.

The subset of parameters controlling the averaged charged hadron multiplicity is the target of our tune. The list contains the parameters $\alpha_{\mathrm{ch}}$ and $\beta_{\mathrm{ch}}$ defined in Eq. (1) and the five PYTHIA parameters discussed before in Sec. II B. The ranges for $\alpha_{\mathrm{ch}}$ and $\beta_{\mathrm{ch}}$ parameters are chosen in a such a way that they cover the values reported by experimental fits; see Table V. The same approach is followed to define the PYTHIA parameters range from the HERMES tune; see Table II.

The impact of each parameter range on the predictions of averaged charge multiplicity for $\nu_{\mu} \mathrm{CC}$ interactions on proton is shown in Fig. 9. As expected, both $\alpha_{\mathrm{ch}}$ and $\beta_{\mathrm{ch}}$ act on low $W$, and their effect vanishes gradually over the transition region. In the PYTHIA region, the largest contribution comes from the Lund $a$ and Lund $b$ parameters. In the transition region, the prediction will be determined by both sets of parameters: as a consequence, we anticipate a correlation between PYTHIA and the low- $W$ AGKY parameters after the fit. The parameter ranges that define the parameter space are defined in Table VII.

\section{CONSTRUCTION OF THE GENIE PREDICTIONS AND EVALUATION OF THE LIKELIHOOD}

To build the hadronization prediction for the data described in Sec. III, $\nu_{\mu}$ and $\bar{\nu}_{\mu} \mathrm{CC}$ events on $\mathrm{H}$ and ${ }^{2} \mathrm{H}$ 

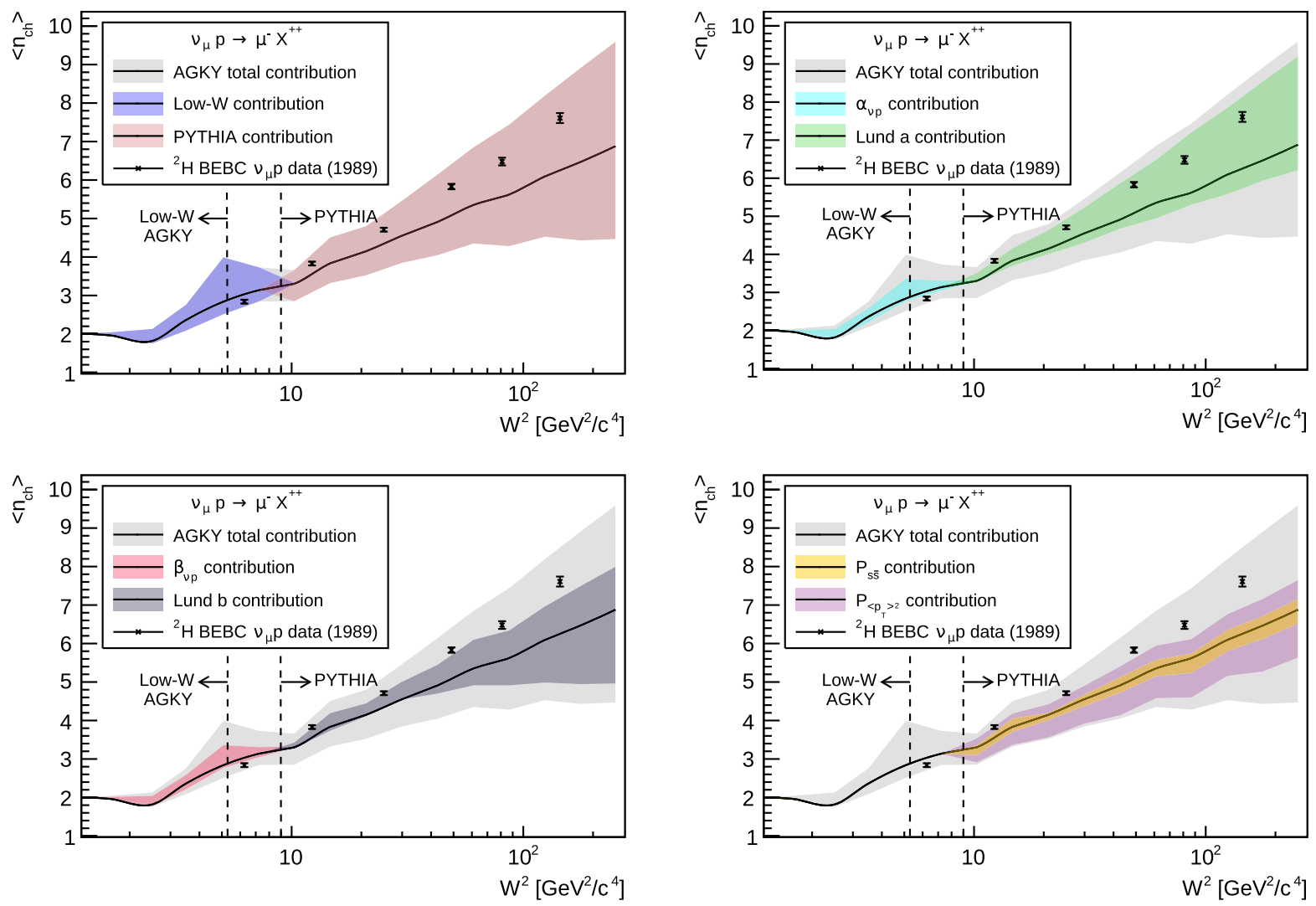

FIG. 9. Impact of fit parameters on the prediction of the averaged charged multiplicity, as a function of $W$, for $\nu_{\mu} p \rightarrow \mu^{-} X^{++}$ interaction on a deuterium target. Each parameter has been varied within the range of study specified in Table VII. The top left plot shows the total contributions from low- $W$ and PYTHIA parameters. All the other plots specify the contribution from specific parameters compared to the total, which is always rendered with the gray area. Dashed lines correspond to $W_{\min }^{\mathrm{tr}}$ and $W_{\max }^{\mathrm{tr}}$, defining the transition region. BEBC data [72] are shown for reference.

TABLE VII. Best-fit result parameters for the AGKY 2021 global tune and ${ }^{2} \mathrm{H}$-only tune. The range of study and priors used in the tune are specified in the table. The 2010 GENIE AGKY parameter values are also specified for reference, as is the parameter name used in the GENIE software. See Secs. VII A and VII B for the details on the error estimation of each tune. Posterior distributions are not always symmetric; in that case, the interval is reported accordingly. The total $\chi^{2}$ obtained from each fit is obtained from the minimization of Eq. (10).

\begin{tabular}{|c|c|c|c|c|c|}
\hline Parameter & GENIE parameter name & 2010 GENIE & Allowed range & 2021 Global fit & $2021{ }^{2} \mathrm{H}$ fit \\
\hline \multicolumn{6}{|c|}{ Low- $W$ empirical model } \\
\hline$\alpha_{\nu p}$ & KNO-Alpha-vp & 0.40 & {$[-1.0,2.0]$} & $1.1 \pm 0.3$ & $1.2 \pm 0.4$ \\
\hline$\alpha_{\nu n}$ & KNO-Alpha-vn & -0.20 & {$[-1.0,2.0]$} & $1.75_{-0.11}^{+0.14}$ & $-0.58 \pm 0.07$ \\
\hline$\alpha_{\bar{\nu} p}$ & KNO-Alpha-vbp & 0.02 & {$[-1.0,2.0]$} & $1.32_{-0.14}^{+0.16}$ & $1.9 \pm 0.08$ \\
\hline$\alpha_{\bar{\nu} n}$ & KNO-Alpha-vbn & 0.80 & {$[-1.0,2.0]$} & $1.11 \pm 0.09$ & $1.07 \pm 0.3$ \\
\hline$\beta_{\nu p}$ & KNO-Beta-vp & 1.42 & {$[0.0,2.5]$} & $0.79 \pm 0.15$ & $0.9 \pm 0.3$ \\
\hline$\beta_{\nu n}$ & KNO-Beta-vn & 1.42 & {$[0.0,2.5]$} & $0.5 \pm 0.1$ & $1.9 \pm 0.3$ \\
\hline$\beta_{\bar{\nu} p}$ & KNO-Beta-vbp & 1.28 & {$[0.0,2.5]$} & $0.8 \pm 0.1$ & $0.3 \pm 0.1$ \\
\hline$\beta_{\bar{\nu} n}$ & KNO-Beta-vbn & 0.95 & {$[0.0,2.5]$} & $0.88_{-0.08}^{+0.09}$ & $0.9 \pm 0.2$ \\
\hline \multicolumn{6}{|c|}{ PYTHIA } \\
\hline$P_{s \bar{s}}$ & PYTHIA-SSBarSuppression & 0.30 & {$[0.0,1.0]$} & $0.27 \pm 0.04$ & $0.29 \pm 0.05$ \\
\hline$\left\langle p_{\perp}^{2}\right\rangle\left(\mathrm{GeV}^{2} / c^{2}\right)$ & PYTHIA-GaussianPt2 & 0.44 & {$[0.1,0.7]$} & $0.46 \pm 0.05$ & $0.43 \pm 0.04$ \\
\hline$E_{\text {Cutoff }}(\mathrm{GeV})$ & PYTHIA-RemainingEnergyCutoff & 0.20 & {$[0.0,1.0]$} & $0.30 \pm 0.04$ & $0.24 \pm 0.05$ \\
\hline Lund $a$ & PYTHIA-Lunda & 0.30 & {$[0.0,2.0]$} & $1.53 \pm 0.13$ & $1.85 \pm 0.15$ \\
\hline \multirow[t]{2}{*}{ Lund $b\left(c^{4} / \mathrm{GeV}^{2}\right)$} & PYTHIA-Lundb & 0.58 & {$[0.0,1.5]$} & $1.16 \pm 0.09$ & $1.0 \pm 0.2$ \\
\hline & & & $\chi^{2}=$ & 87.9/62 DoF & 29.5/32 DoF \\
\hline
\end{tabular}


are simulated. Events are generated using a " $1 / E$ "-like flux, with a $0.1-200 \mathrm{GeV}$ energy range. This is sufficient as the observables are given in terms of $W$; hence, the neutrino flux is factorized out.

To compute the prediction associated to the $i$ th dataset from Tables III and IV, we select events simulated with the neutrino flux and target of the corresponding experiment and processed using the same experimental cuts. For each selected event, we reconstruct $E_{\nu}$ and $W$ following the recipes described in Sec. III. The events are classified in bins according to the reconstructed $W$, and for each bin, we evaluate the average charged multiplicity $\left\langle n_{\mathrm{ch}}\right\rangle_{i}(W)$. This operation is repeated for a number of points in the parameter space $\boldsymbol{\theta}$ defined in Table VII. Each experiment has a different binning system, and therefore we identify the $W$ bins using two indices: one for the dataset (i) and the other one for the bin index inside the dataset $(j)$. Labeling with $\boldsymbol{\theta}$ the vector of coordinates of a point belonging to the parameters space, we can define our predictions associated to the $i$ th dataset and a given $j$ th $W$ bin as $\left\langle n_{\mathrm{ch}}\right\rangle_{i}\left(W_{i j} \mid \boldsymbol{\theta}\right)$. The statistical error due to the MC sample size is also evaluated, and this is referred to as $\sigma_{i j}(\boldsymbol{\theta})$.

We use Professor [85] to generate a parametrization denoted as $\tilde{n}_{i j}(\boldsymbol{\theta})$ and $\tilde{\sigma}_{i j}(\boldsymbol{\theta})$ interpolating the values of $\left\langle n_{\mathrm{ch}}\right\rangle_{i}\left(W_{i j} \mid \boldsymbol{\theta}\right)$ and $\sigma_{i j}(\boldsymbol{\theta})$ as a function of $\boldsymbol{\theta}$. The parametrization is a generic polynomial of order $M$ in the $P$-dimensional space [85], whose analytical form is

$$
\begin{aligned}
\tilde{n}_{i j}(\boldsymbol{\theta})= & \alpha_{0}^{i j k}+\sum_{n=1}^{P} \beta_{n}^{i j k} \theta_{n}+\sum_{n \leq m} \gamma_{n m}^{i j k} \theta_{n} \theta_{m} \\
& +\cdots+\sum_{n_{1} \leq \ldots \leq n_{M}} \xi_{n_{1} \ldots n_{M}}^{i j k} \prod_{\ell=1}^{M} \theta_{n_{\ell}}
\end{aligned}
$$

where $\theta_{n}$ is the coordinate of the $n$-th parameter and $M$ is the polynomial order, set to fourth order in this work. The coefficients $\alpha_{0}^{i j k}, \beta_{n}^{i j k}, \gamma_{(n m)}^{i j k}, \ldots, \xi_{\left(n_{1} \ldots n_{M}\right)}^{i j k}$ are determined by Professor fitting the parametrization against the computed $\left\langle n_{\mathrm{ch}}\right\rangle_{i}\left(W_{i j} \mid \boldsymbol{\theta}\right)$ obtained by generating $\mathrm{O}\left(10^{4}\right)$ points uniformly spread within parameter space defined in Table VII. Nonphysical regions in the sampled parameter space are avoided by applying a veto function. In particular, every combination of $\boldsymbol{\theta}$ has to verify that $\left\langle n_{\mathrm{ch}}\right\rangle \geq 0$ at the pion production threshold. The parametrization $\tilde{n}_{i j}(\boldsymbol{\theta})$ is used instead of the exact predictions in order to estimate the bestfit parameters by minimizing the $\chi^{2}$. The main advantage of this method is the reduction of the brute-force scans computational complexity while allowing for massive parallelization.

\section{A. Professor interpolation cutoff condition}

As mentioned in Sec. IV C, in view of the fact that the Professor interpolation is just an approximation, it can fail to describe the actual prediction. When this happens, we

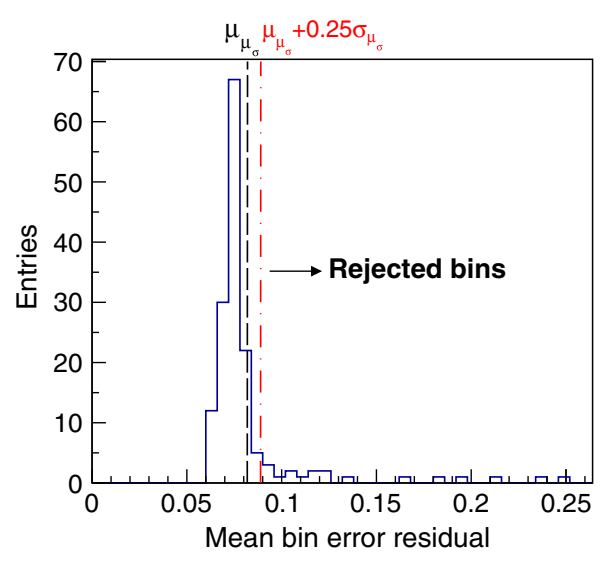

FIG. 10. Distribution of mean bin error residual for all data points. The distribution mean value $\left(\mu_{\mu_{\sigma}}\right)$ is shown with a dashed black line. Data points with a mean value higher than 0.25 of the mean bin error variance $\left(\sigma_{\mu_{\sigma}}\right)$ are rejected. This cutoff value is shown with the dashed red line.

remove from the analysis data points whose Professor interpolation, of the predicted mean value or predicted error, disagree too much with the GENIE prediction corresponding to that data point. The relative difference between the interpolation and the GENIE prediction is known as residual. For each data point, we calculate the bin central value and bin error residuals for all the points in our parameter space. The distributions of the residual for central values and errors are monitored, and whenever the means or the variances of a bin are too far from the average values among all bins, the corresponding data point is removed. In this analysis, the cutoff condition requires that any data points with a mean central value or error that exceeds the average values among all bins by $0.25 \sigma_{\mu_{\mu}}$ or $0.25 \sigma_{\mu_{\sigma}}$, respectively, are removed from the analysis. An example of the cutoff condition on the error residual distribution for all data points is shown in Fig. 10.

The corresponding distribution associated to the bin central value and bin error residual for the last bin of the BEBC [67] and the two lasts bins of the FNAL $15 \mathrm{ft}$ [38] datasets are shown in Figs. 11(b) and 11(c) and Figs. 11(e) and 11(f), respectively. Two examples are given in Fig. 11: a dataset in which the interpolation is accurate for all the $W^{2}$ range and a dataset in which the interpolation fails for some of the dataset points, highlighted with empty markers. This criteria allow us to ensure that the Professor parametrization does not fail for the data considered in the tune. A total of $\sim 18 \%$ of the data points have been removed due to this requirement. In this work, it has been observed that the residual variance increases with $W^{2}$, with few exceptions. The complete list of removed data points is specified in Sec. VII.

The variance of the residual distribution for a given data point can be improved by increasing the order of the polynomial used for the Professor interpolation. In this case, a polynomial of order 4 is used. However, specifically 


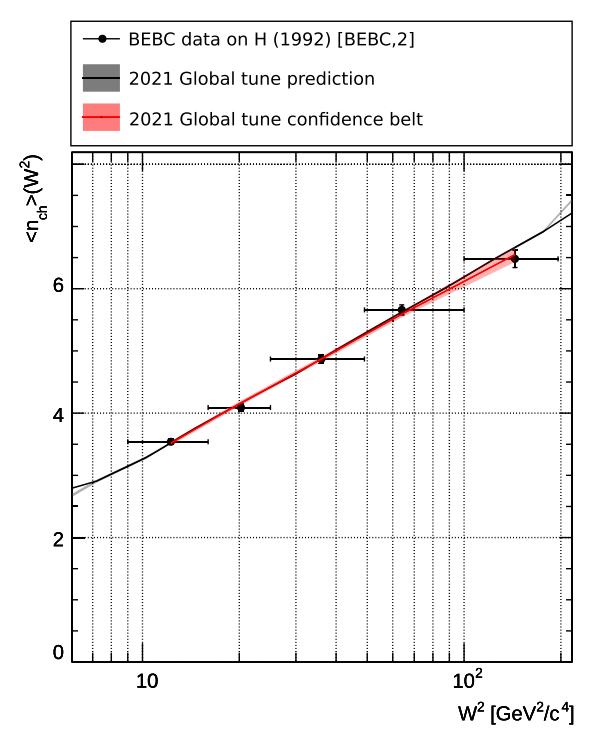

(a)

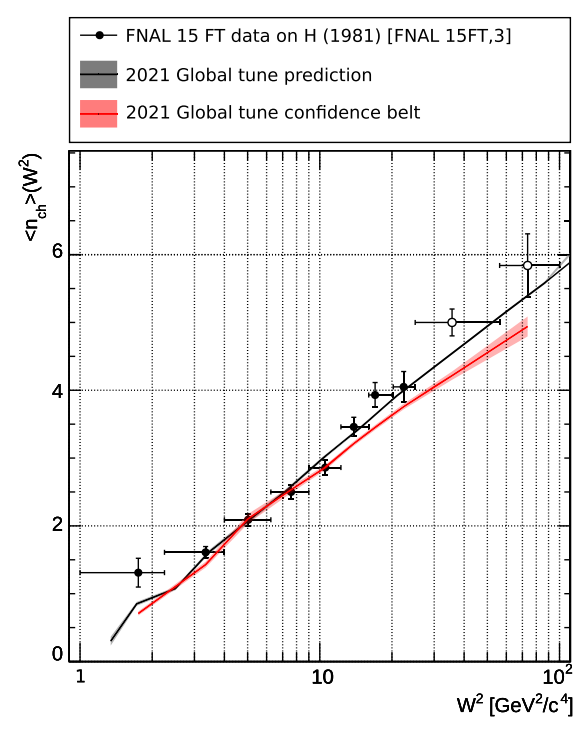

(d)

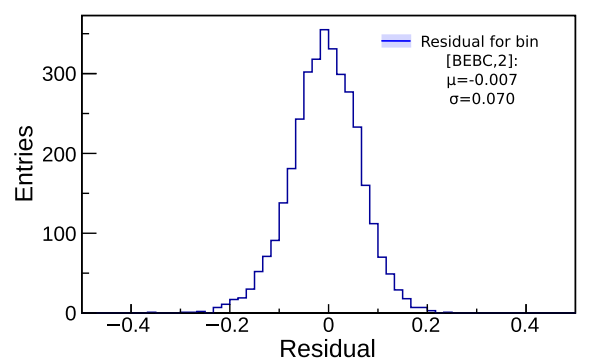

(b)

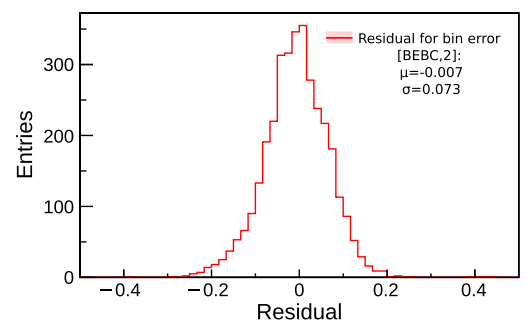

(c)

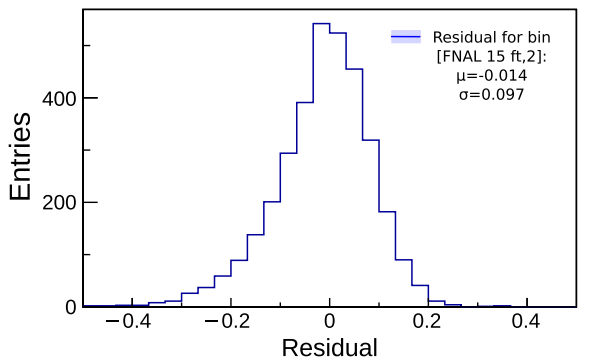

(e)

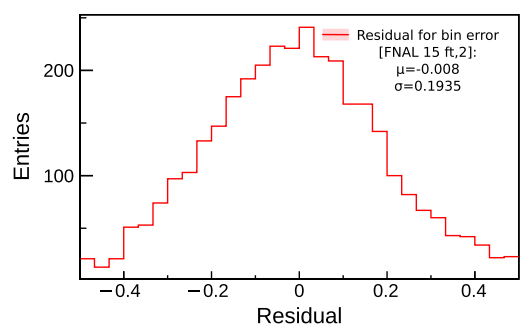

(f)

FIG. 11. Comparisons of $\nu_{\mu}$ data on $\mathrm{H}$ against predictions obtained from the Professor parametrization (red confidence belt) at the best-fit value for the AGKY global tune and the actual GENIE prediction (black line). Data points that do not satisfy our selection criteria are shown in empty markers. For the Professor parametrization, the uncertainties of the tuned parameters are propagated to the prediction considering the full covariance matrix. For three selected bins [the bin with highest $W$ for $(\mathrm{BEBC}, 2)(\mathrm{b}$ and $\mathrm{c})$ and the two higher $W$ bins for the (FNAL 15FT,3) plot (e and f) on the left column], the central values and error residual distributions are shown, blue and red, respectively: accepted parametrizations at the top and rejected parametrization at the bottom. It can be seen that the residual distribution of the rejected bins is wider than its accepted counterpart. In this particular case, the two data points with higher $W$ are neglected as the parametrization of the bin value and error do not satisfy the required criteria. (a) Dataset with good interpolations, (b) accepted central value residual distribution, (c) accepted error residual distribution, (d) dataset with some rejected interpolations, (e) Rejected central value residual distribution, and (f) rejected error residual distribution.

in this particular tune where 13 parameters are tuned, an increase of the order requires the generation of a much higher number of MC samples, which can be very computationally demanding.

\section{B. Parameter priors}

Our parameters of interest affect other hadronization observables and not only the averaged multiplicity. This is taken into account by using Gaussian priors. 


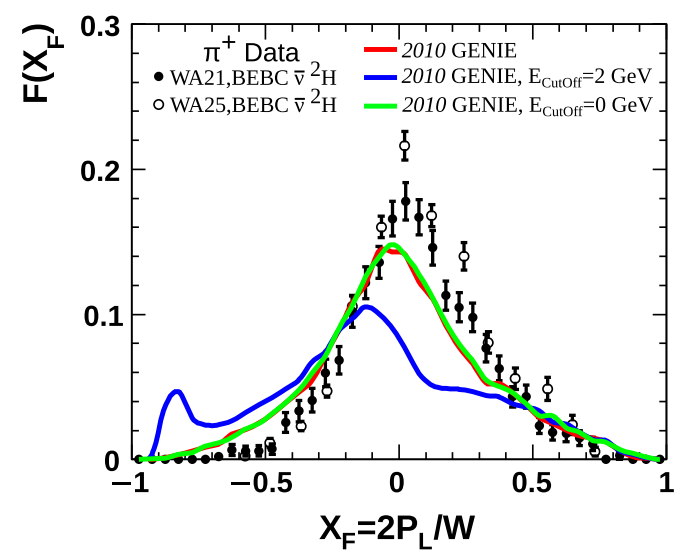

(a)

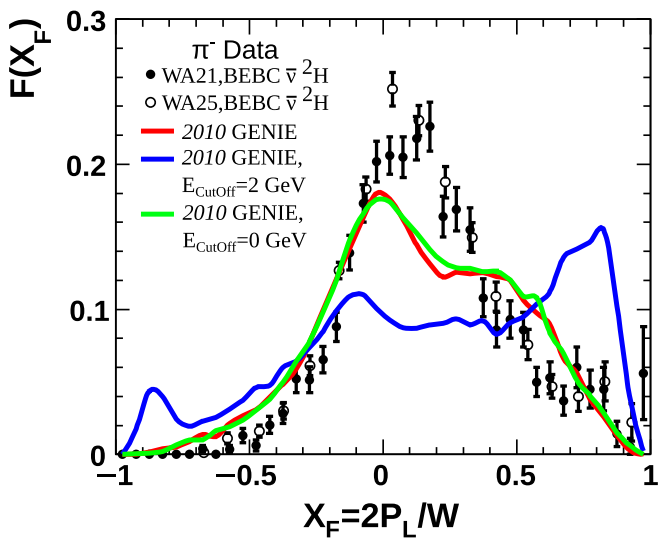

(b)

FIG. 12. Effect of $E_{\text {Cutoff }}$ on the $x_{\mathrm{F}}$ invariant distributions for $\pi^{+}$(a) and $\pi^{-}$(b) in $\bar{\nu}_{\mu}$ data on ${ }^{2} \mathrm{H}$ from the BEBC experiment [73]. The 2010 GENIE tune value for the energy cutoff is $E_{\text {Cutoff }}=0.2 \mathrm{GeV}$.

The $s \bar{s}$ suppression factor not only impacts the averaged multiplicity data but also the $\eta$ multiplicity production; see Fig. 4. A prior of $0.30 \pm 0.05$ is considered in order to preserve a good agreement with the SKAT data [63].

Variations of $E_{\text {Cutoff }}$ affect the shape of $F\left(x_{\mathrm{F}}\right)$ invariant distribution, defined as

$$
F\left(x_{\mathrm{F}}\right)=\frac{1}{N_{\mathrm{ev}}} \cdot \frac{1}{\pi} \cdot \frac{E}{p^{L \max c}} \cdot \frac{d N}{d x_{\mathrm{F}}},
$$

where $x_{\mathrm{F}}$ is the Feynman variable, $N_{\mathrm{ev}}$ is the total number of events, and $E$ and $p^{L \max }$ are the energy and maximum longitudinal momentum of the final-state hadron in the hadronic center of mass, respectively. The $F\left(x_{\mathrm{F}}\right)$ invariant distribution describes the fragmentation process for the forward and backward hemispheres, and it allows us to study the symmetry between this two fragmentation regions. In Fig. 12, the $F\left(x_{\mathrm{F}}\right)$ invariant distribution for $\bar{\nu}_{\mu}$ data on ${ }^{2} \mathrm{H}$ [73] is compared against GENIE predictions obtained by varying the $E_{\text {CutOff }}$ within a $[0,2] \mathrm{GeV}$ range. The main conclusion is that small values of this parameter preserve the agreement with data. To avoid an increase of $F\left(x_{\mathrm{F}}\right)$ at $\left|x_{\mathrm{F}}\right| \sim 1$, we apply a prior on $E_{\text {CutOff }}$ of $0.25 \pm 0.05 \mathrm{GeV}$.

Another parameter that has a strong impact on other observables is $\left\langle p_{\perp}^{2}\right\rangle$. As demonstrated in Fig. 5, low values of $\left\langle p_{\perp}^{2}\right\rangle$ are not in agreement with data for $\left\langle p_{T}^{2}\right\rangle$ distributions. Thus, we also apply a prior on the parameter to guarantee the agreement with these data of $0.44 \pm 0.05(\mathrm{GeV} / c)^{2}$. No priors are applied to the remaining parameters.

\section{Final form of the $\chi^{2}$}

Using the parametrization and the corresponding set of points belonging to the $i$ th dataset, $\mathcal{D}_{i j} \pm \delta \mathcal{D}_{i j}$, we seek to estimate the best-fit parameters $\hat{\boldsymbol{\theta}}$ by minimizing the quantity:

$$
\chi^{2}(\boldsymbol{\theta})=\sum_{i, j} w_{i j} \frac{\left(\tilde{n}_{i j}(\boldsymbol{\theta})-\mathcal{D}_{i j}\right)^{2}}{\tilde{\sigma}_{i j}^{2}(\boldsymbol{\theta})+\delta \mathcal{D}_{i j}^{2}}+\sum_{l} \frac{\left(\theta_{l}-\mu_{l}\right)^{2}}{\sigma_{l}^{2}} .
$$

The first term allows the minimization between data and prediction while applying weights, $w_{i j}$, that allow us to consider only specific data points in the fit. The second term adds uncorrelated Gaussian priors for a given parameter; the vectors of central values and variances are denoted $\mu_{l}$ and $\sigma_{l}$, respectively.

\section{AGKY TUNE RESULTS}

Starting from $\nu_{\mu}$ and $\bar{\nu}_{\mu}$ hadronization data, two tunes were considered: a global tune (2021 GENIE global) and a deuterium-only tune $\left(2021^{2} \mathrm{H}\right)$. The reason for a deuteriumonly fit is because other studies showed tensions between data on $\mathrm{H}$ and ${ }^{2} \mathrm{H}$ targets on bubble chamber experiments [42]. The goal of the global tune is to improve the agreement with hydrogen and deuterium targets, regardless of these tensions, while the deuterium-only tune was performed to quantify the tensions within the same framework. A hydrogen-only fit was not considered because it could not constrain the neutron related parameters of the low- $W$ empirical model.

The analyses procedure outlined in the previous sections was applied to both tunes. The likelihood function, Eq. (10), was minimized against the averaged charged multiplicity data that satisfy our selection criteria; see Sec. III. The best-fit parameter set for both tunes and the $\chi^{2}$ values obtained using the Professor parametrizations and Eq. (10) are summarized in Table VII.

GENIE predictions for all the averaged charged multiplicity data available are shown in Figs. 13-16 before and after the AGKY tunes. The results show the prediction for the 2021 GENIE global tune in red and 2021 GENIE $^{2} \mathrm{H}$ tune in green. To distinguish data points used in the analyses 


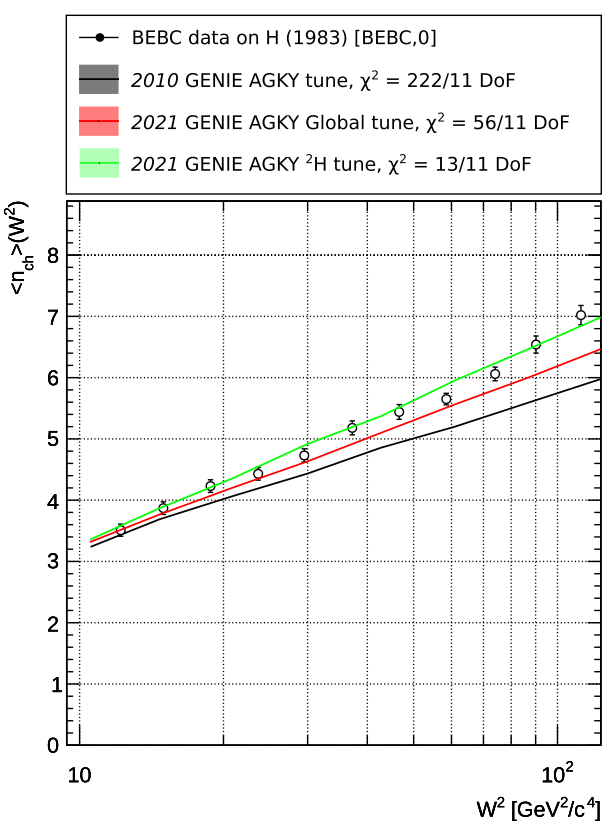

(a)

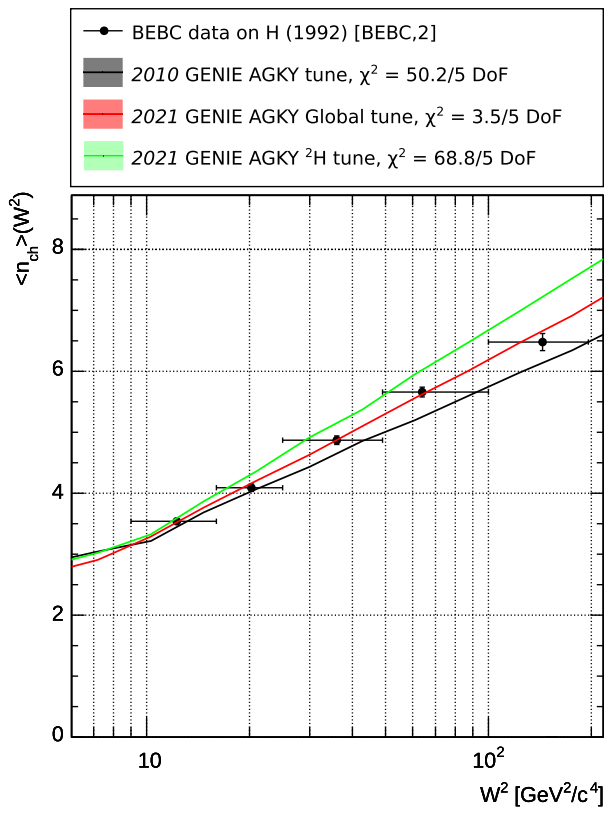

(c)

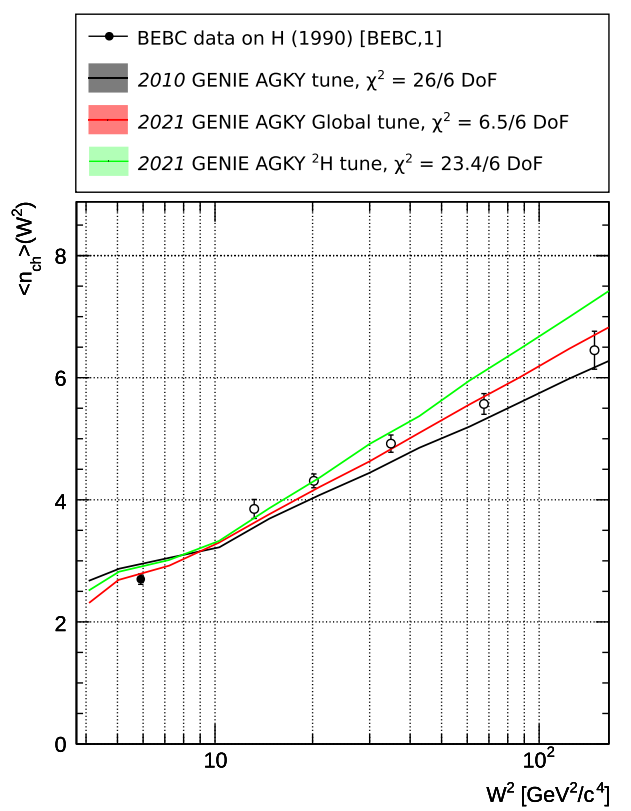

(b)

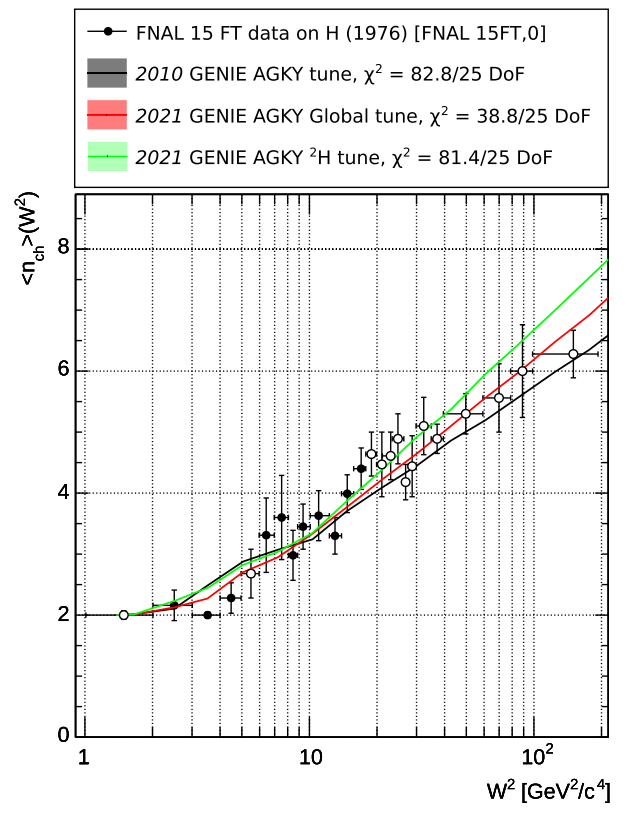

(d)

FIG. 13. Comparison of $\left\langle n_{\mathrm{ch}}\right\rangle$ against neutrino-induced hadronization data on $\nu_{\mu}+p$ interactions on $\mathrm{H}$ from BEBC [65-67] and FNAL [64] bubble chamber experiments filled with H. Data points used in the AGKY 2021 global tune analysis are shown as filled black markers. Discarded data points are represented using empty markers. The ${ }^{2} \mathrm{H}$ tune prediction is shown for comparison only. The predictions are computed using the parameters specified in Table VII. The $\chi^{2}$ values are calculated against all the data from each experiment. See the definition of tags in Table VIII.

from those that were not, the used points have completely black markers, the others are represented as empty circles. Each dataset is associated to a tag, defined in Table VIII. Vertical error bars include statistical and systematic uncertainties following our data review. Horizontal bars correspond to the bin width used in the data release and are only shown if those are available in the original paper.
In terms of prediction differences, the 2021 GENIE global tune tends to underpredict deuterium data, whereas the 2021 GENIE $^{2} \mathrm{H}$ tune overpredicts the hydrogen data. This is especially true for the PYTHIA region, at high $W$. This is translated in the parameters with an increase (decrease) of Lund $a$ (Lund $b$ ) for the deuterium tune with respect to the global tune. 


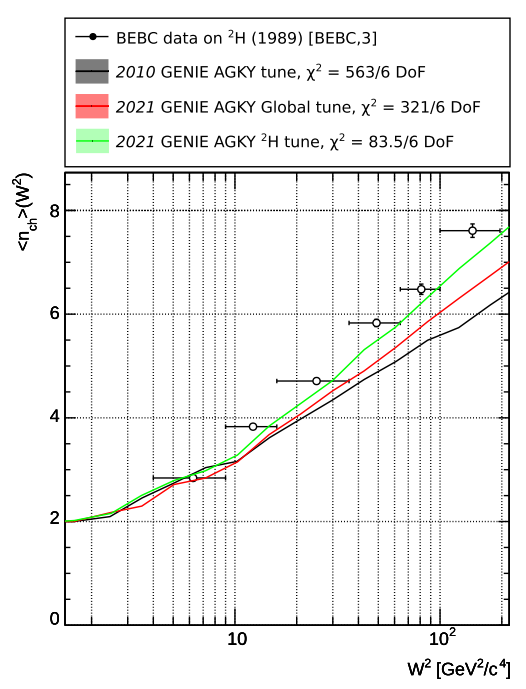

(a) $\nu_{\mu}+p \rightarrow \mu^{-} X^{++}$

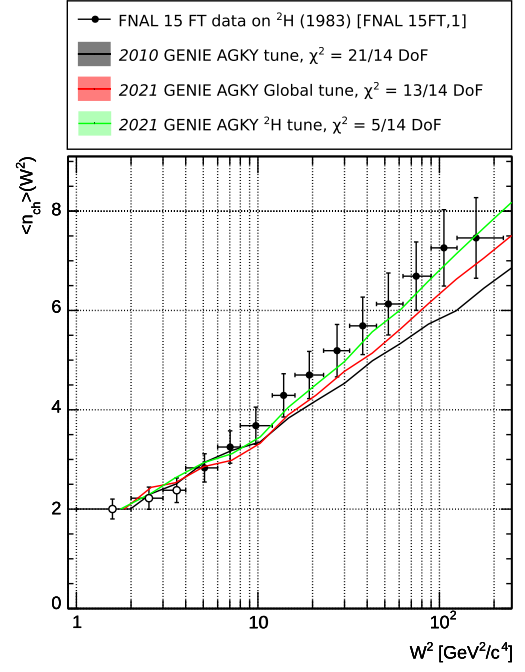

(b) $\nu_{\mu}+p \rightarrow \mu^{-} X^{++}$

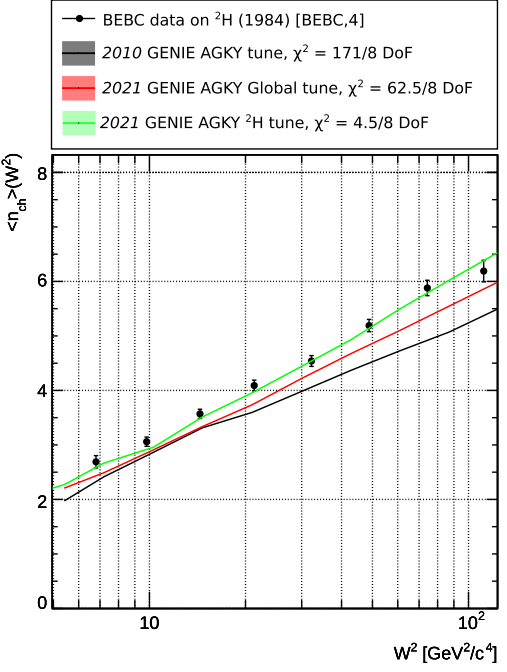

(c) $\nu_{\mu}+n \rightarrow \mu^{-} X^{+}$

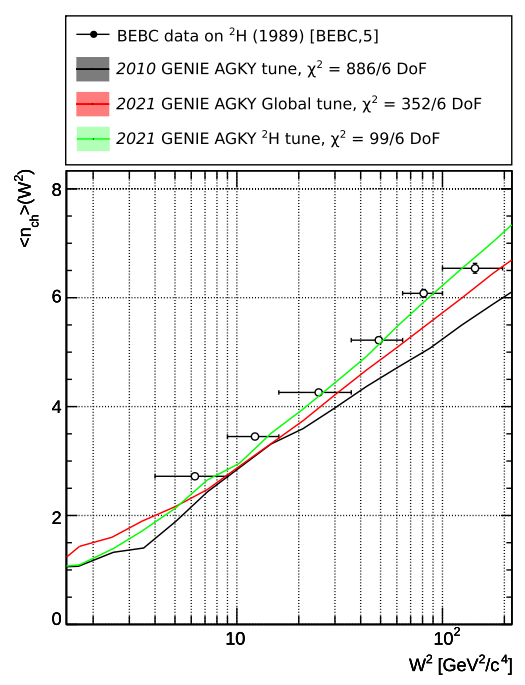

(d) $\nu_{\mu}+n \rightarrow \mu^{-} X^{+}$

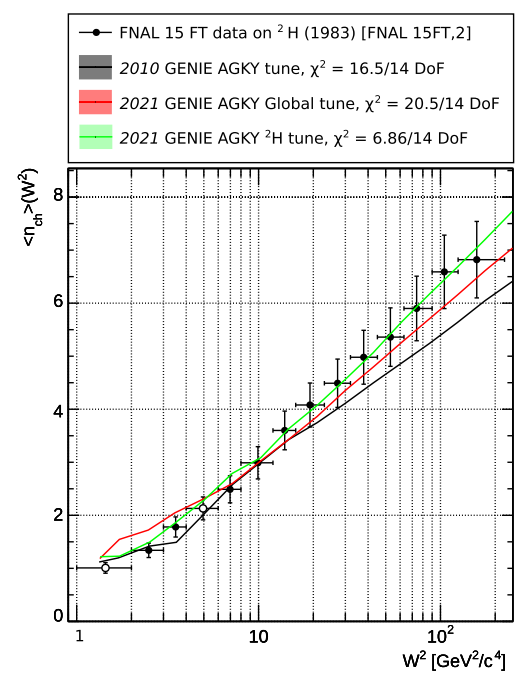

(e) $\nu_{\mu}+n \rightarrow \mu^{-} X^{+}$

FIG. 14. Comparison of $\left\langle n_{\mathrm{ch}}\right\rangle$ against neutrino-induced hadronization data on $\nu_{\mu}$ interactions on proton (p) and neutron (n) from the BEBC bubble chamber experiment filled with ${ }^{2} \mathrm{H}[39,72]$. Data points used in the AGKY 2021 global tune analysis are shown as filled black markers. Discarded data points are represented using empty markers. The predictions are computed using the parameters specified in Table VII. The $\chi^{2}$ values are calculated against all the data from each experiment. See the definition of tags in Table VIII.

The summary of the $\chi^{2}$ values per dataset as well as the total contributions are shown in Table IX. Three different $\chi^{2}$ values are presented: $\chi_{2010}^{2}, \chi_{2021(\text { Global })}^{2}$, and $\chi_{2021\left({ }^{2} \mathrm{H}\right)}^{2}$ using, respectively, the 2010 GENIE, 2021 GENIE global, and 2021 GENIE ${ }^{2} \mathrm{H}$ tune parameters. The $\chi^{2}$ values per dataset are computed by comparing the GENIE predictions against all the data points in each dataset, regardless of the point being used in the fit or not. Differences between the $\chi^{2}$ obtained with Eq. (10) and the one calculated using the GENIE predictions directly are expected. One of the reasons is that Eq. (10) only considers the data points included in the tune. Moreover, further differences arise form the fact that the Professor parametrization $\tilde{n}_{i j}$ is not exact, as explained in Sec. VI.

It is important to stress that the total $\chi^{2}$ from Table IX is not providing any information related to goodness of fit, but it simply shows the general agreement with respect to available datasets. A sense of the goodness of fit can be obtained looking at the total $\chi^{2}$ calculated with the datasets included in the fit only; see Table X.

The parameters covariance matrices for both tunes are obtained by inverting the Hessian of the log-likelihood function at the best fit point; see Tables XI and XII. As expected, the low- $W$ AGKY and PYTHIA parameters are now correlated in both tunes because of the interplay 


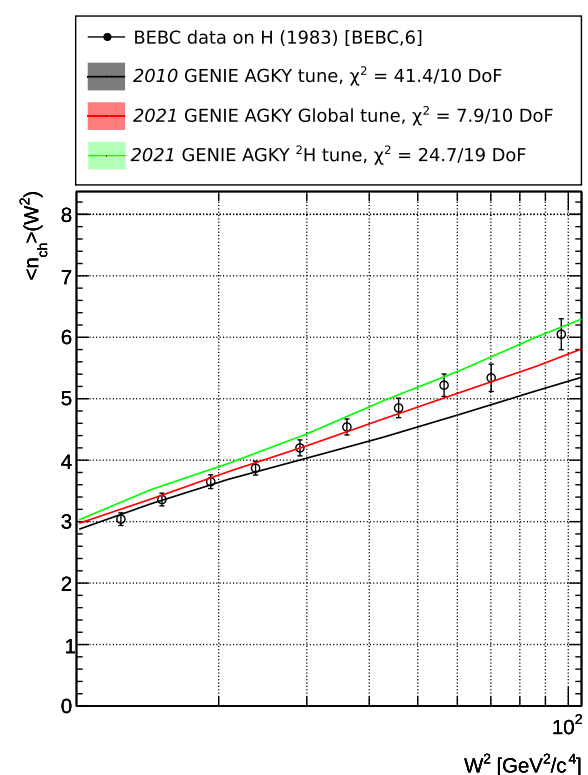

(a)

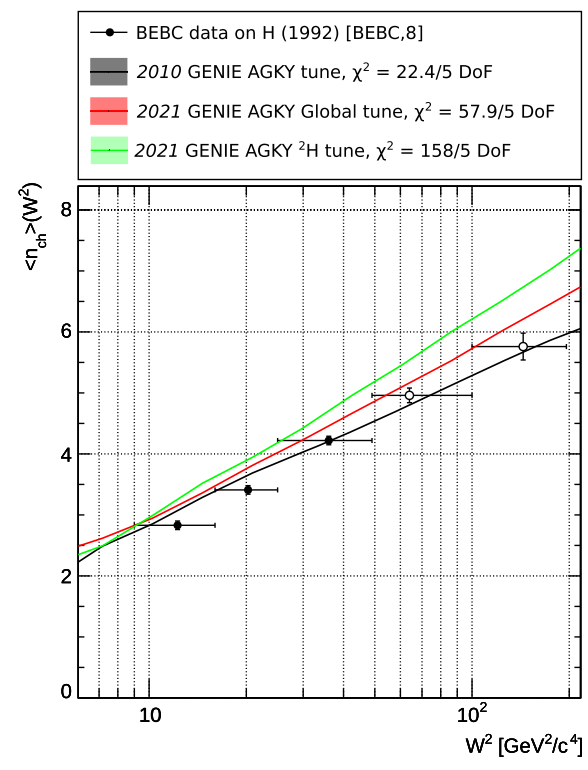

(c)

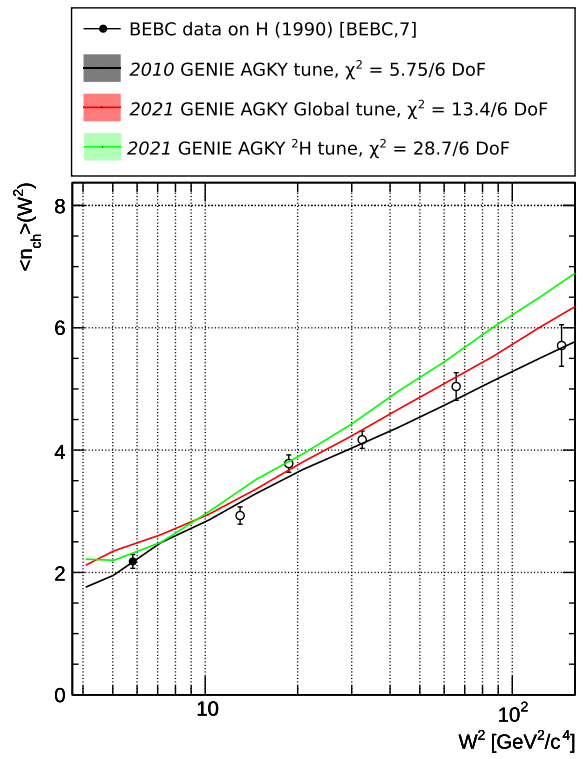

(b)

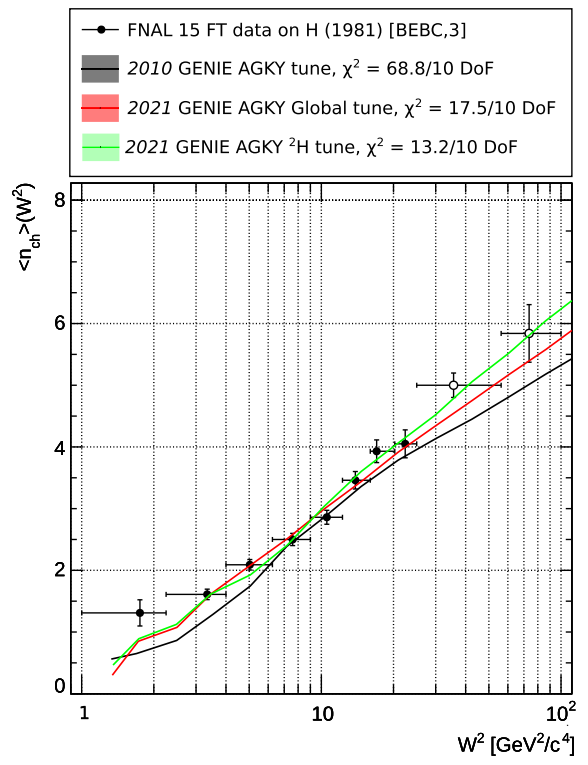

(d)

FIG. 15. Comparison of $\left\langle n_{\mathrm{ch}}\right\rangle$ against neutrino-induced hadronization data on $\bar{\nu}_{\mu}+p$ interactions on $\mathrm{H}$ from the BEBC [65-67] and FNAL [68] bubble chamber experiment filled with H. Data points used in the AGKY 2021 global tune analysis are shown as filled black markers. Discarded data points are represented using empty markers. The predictions are computed using the parameters specified in Table VII. The $\chi^{2}$ values are calculated against all the data from each experiment. See the definition of tags in Table VIII.

of the models in the transition region, with a number of parameters showing a correlation above 50\%. See a graphical representation of the correlation matrix in Fig. 17.

The results form the 2021 GENIE AGKY tunes will available in GENIE v3.2.0. Users can run the 2021 GENIE tunes global and ${ }^{2} \mathrm{H}$ tunes out of the box using the G18_02a_03_330 and G18_02a_03_320 comprehensive configurations, respectively.

\section{A. 2021 GENIE AGKY global tune}

After the AGKY global tune, the GENIE predictions show better agreement with the data. In particular, for the datasets included in the 2021 GENIE global tune, the $\chi^{2}$ associated to the prediction is $\chi_{2010}^{2}=486 / 109$ degrees of freedom (DoF). After the tune, the $\chi_{2021(\text { Global) }}^{2}$ is $242 / 109$ DoF. This is clearly an improvement, although the agreement is not completely satisfactory since the $\mathrm{p}$ value is $4 \times 10^{-12}$. 


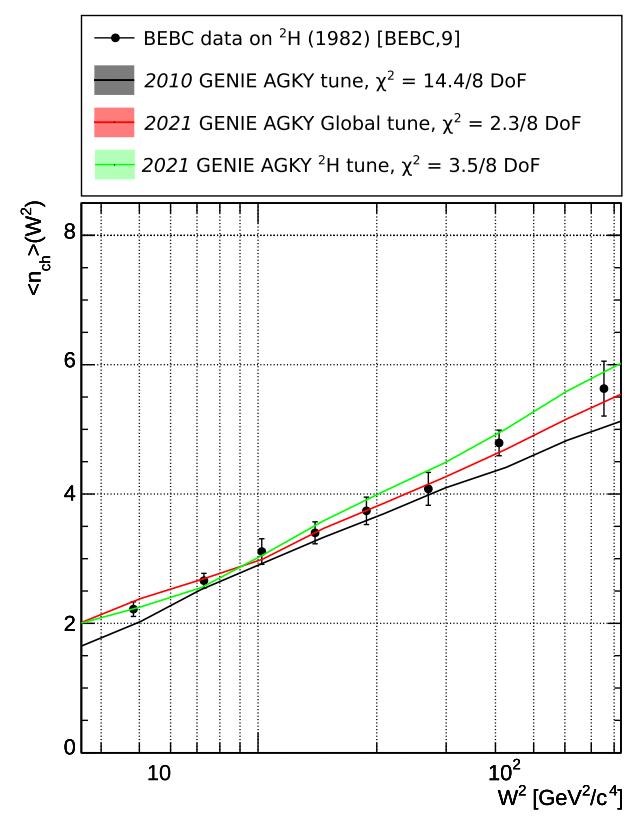

(a) $\bar{\nu}_{\mu}+p \rightarrow \mu^{+} X^{0}$

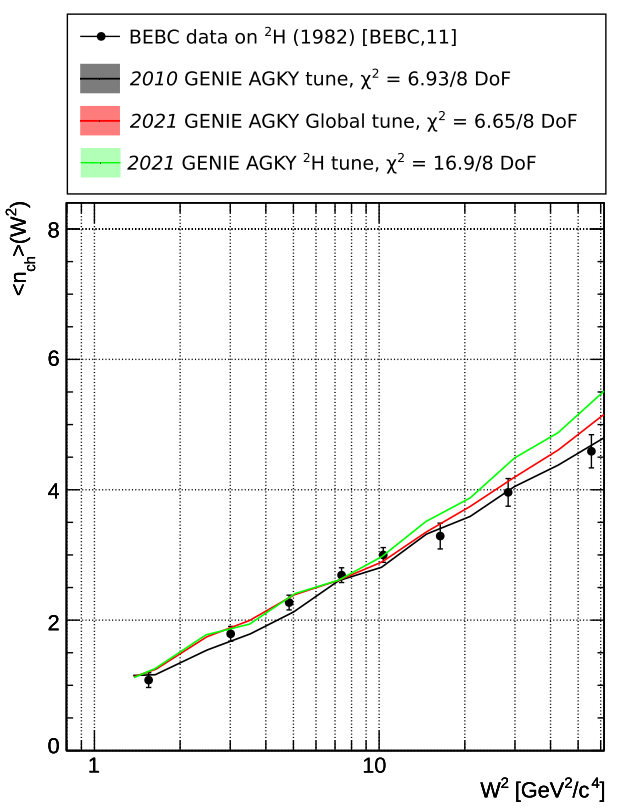

(c) $\bar{\nu}_{\mu}+n \rightarrow \mu^{+} X^{-}$

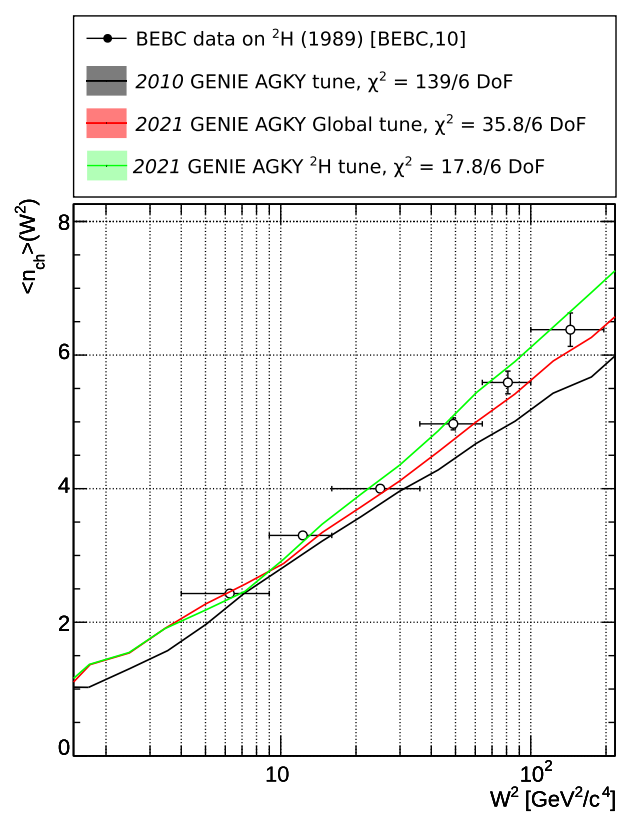

(b) $\bar{\nu}_{\mu}+p \rightarrow \mu^{+} X^{0}$

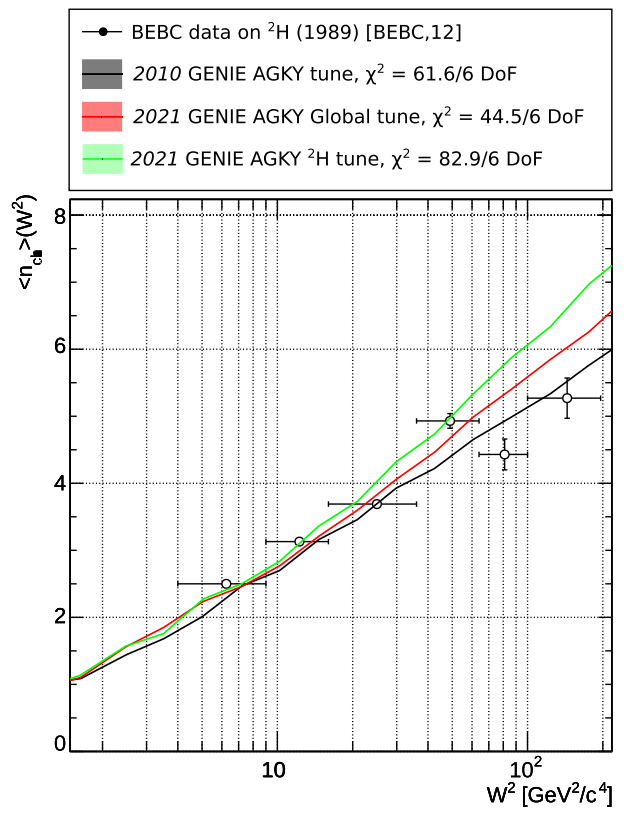

(d) $\bar{\nu}_{\mu}+n \rightarrow \mu^{+} X^{-}$

FIG. 16. Comparison of $\left\langle n_{\mathrm{ch}}\right\rangle$ against neutrino-induced hadronization data on $\bar{\nu}_{\mu}$ interactions on proton (p) and neutron (n) from the BEBC bubble chamber experiment filled with ${ }^{2} \mathrm{H}[39,72]$. Data points used in the AGKY 2021 global tune analysis are shown as filled black markers. Discarded data points are represented using empty markers. The predictions are computed using the parameters specified in Table VII. The $\chi^{2}$ values are calculated against all the data from each experiment. See the definition of tags in Table VIII.

The improvement in the data description is general, and both deuterium and hydrogen samples have better agreement. Moreover, after the tune, both hydrogen and deuterium samples have similar goodness of fit, and in general, the level of agreement is the same. This can be noted from the $\chi^{2}$ contributions from Table $\mathrm{X}$.
The agreement with the datasets not included in the tune has also improved, as shown in Table IX. The total $\chi^{2}$ computed using all available data is reduced significantly for both $\mathrm{H}$ and ${ }^{2} \mathrm{H}$ datasets. Particularly, the global tune shows better agreement against all hydrogen data. As expected from Sec. IV C, the datasets with highest 
TABLE VIII. Summary of data used for comparisons in Figs. 7, 14, 15, and 16. This table links the experiment and the tag used for the legend in each plot to the corresponding reference.

\begin{tabular}{|c|c|c|c|}
\hline Experiment & Target & Tag & References \\
\hline \multicolumn{4}{|c|}{$\nu_{\mu}+p \rightarrow \mu^{-} X^{++}$} \\
\hline FNAL 15 ft (1976) & $\mathrm{H}$ & FNAL $15 \mathrm{ft}, 0$ & [64] \\
\hline BEBC (1983) & $\mathrm{H}$ & $\mathrm{BEBC}, 0$ & {$[65]$} \\
\hline BEBC (1990) & $\mathrm{H}$ & $\mathrm{BEBC}, 1$ & [66] \\
\hline BEBC (1992) & $\mathrm{H}$ & $\mathrm{BEBC}, 2$ & [67] \\
\hline FNAL 15 ft (1983) & ${ }^{2} \mathrm{H}$ & FNAL $15 \mathrm{ft}, 1$ & [38] \\
\hline BEBC (1989) & ${ }^{2} \mathrm{H}$ & BEBC,3 & [72] \\
\hline \multicolumn{4}{|c|}{$\nu_{\mu}+n \rightarrow \mu^{-} X^{+}$} \\
\hline FNAL 15 ft (1983) & ${ }^{n} \mathrm{H}$ & FNAL $15 \mathrm{ft}, 2$ & {$[38]$} \\
\hline BEBC (1984) & ${ }^{2} \mathrm{H}$ & BEBC,4 & [73] \\
\hline BEBC (1989) & ${ }^{2} \mathrm{H}$ & $\mathrm{BEBC}, 5$ & [72] \\
\hline \multicolumn{4}{|c|}{$\bar{\nu}_{\mu}+p \rightarrow \mu^{+} X^{0}$} \\
\hline FNAL 15 ft (1981) & ${ }^{\mu} \mathrm{H}$ & FNAL $15 \mathrm{ft}, 3$ & [68] \\
\hline BEBC (1983) & $\mathrm{H}$ & $\mathrm{BEBC}, 6$ & [65] \\
\hline BEBC (1990) & $\mathrm{H}$ & BEBC,7 & [66] \\
\hline BEBC (1992) & $\mathrm{H}$ & $\mathrm{BEBC}, 8$ & [67] \\
\hline BEBC (1982) & ${ }^{2} \mathrm{H}$ & BEBC,9 & [39] \\
\hline BEBC (1989) & ${ }^{2} \mathrm{H}$ & $\mathrm{BEBC}, 10$ & {$[72]$} \\
\hline \multicolumn{4}{|c|}{$\bar{\nu}_{\mu}+n \rightarrow \mu^{+} X^{-}$} \\
\hline BEBC (1982) & ${ }^{2} \mathrm{H}$ & BEBC,11 & [39] \\
\hline BEBC (1989) & ${ }^{2} \mathrm{H}$ & $\mathrm{BEBC}, 12$ & [72] \\
\hline
\end{tabular}

TABLE IX. Summary of $\chi^{2}$ values for the datasets shown in Figs. $13,14,15$, and 16 . The table shows the $\chi^{2}$ per dataset and interaction channel as well as the total and per channel $\chi^{2}$. The $\chi^{2}$ values are calculated using the GENIE predictions for each tune: 2010 GENIE, $\chi_{2010}^{2}, 2021$ GENIE, $\chi_{2021 \text { (Global) }}^{2}$, and 2021 GENIE, $\chi_{2021\left({ }^{2} \mathrm{H}\right)}^{2}$

\begin{tabular}{lccccc}
\hline \hline Experiment & $\chi_{2010}^{2}$ & $\chi_{2021(\mathrm{Global})}^{2} \chi_{2021\left({ }^{2} \mathrm{H}\right)}$ & $\begin{array}{c}\text { Degrees of } \\
\text { freedom }\end{array}$ & $\begin{array}{c}\text { In } \\
\text { tune }\end{array}$ \\
\hline \multicolumn{5}{c}{$\nu_{\mu}+p \rightarrow \mu^{-} X^{++}$} \\
\hline Data on hydrogen \\
\hline FNAL 15 ft,0 & 83 & 39 & 81 & 25 & Partially \\
BEBC,0 & 222 & 56 & 13 & 11 & $\boldsymbol{x}$ \\
BEBC,1 & 26 & 7 & 23 & 6 & Partially \\
BEBC,2 & 50.2 & 3.5 & 68.8 & 5 & $\checkmark$ \\
\hline \multicolumn{5}{c}{ Data on deuterium } \\
\hline FNAL 15 ft,1 & 21 & 13 & 5 & 14 & Partially \\
BEBC,3 & 563 & 321 & 84 & 6 & $\boldsymbol{X}$ \\
Total for $\nu_{\mu} p$ & $\mathbf{9 6 5}$ & $\mathbf{4 4 7}$ & $\mathbf{2 7 5}$ & $\mathbf{6 7}$ & \\
\hline & \multicolumn{5}{c}{$\nu_{\mu}+n \rightarrow \mu^{-} X^{+}$} \\
FNAL 15 ft,2 & 17 & 21 & 7 & 14 & Partially \\
BEBC,4 & 171 & 6 & 5 & 8 & $\checkmark$ \\
BEBC,5 & 886 & 352 & 99 & 6 & $\boldsymbol{x}$ \\
Total for $\nu_{\mu} n$ & $\mathbf{1 , 0 7 4}$ & $\mathbf{4 3 5}$ & $\mathbf{1 1 1}$ & $\mathbf{2 8}$ & \\
\hline
\end{tabular}

(Table continued)
TABLE IX. (Continued)

\begin{tabular}{|c|c|c|c|c|c|}
\hline Experiment & $\chi_{2010}^{2}$ & $\chi_{2021(\text { Global })}^{2}$ & $\chi_{2021\left({ }^{2} \mathrm{H}\right)}$ & $\begin{array}{l}\text { Degrees of } \\
\text { freedom }\end{array}$ & $\begin{array}{l}\text { In } \\
\text { tune }\end{array}$ \\
\hline \multicolumn{6}{|c|}{$\bar{\nu}_{\mu}+p \rightarrow \mu^{+} X^{0}$} \\
\hline \multicolumn{6}{|c|}{ Data on hydrogen } \\
\hline FNAL $15 \mathrm{ft}, 3$ & 69 & 18 & 13 & 10 & Partially \\
\hline BEBC,6 & 41 & 8 & 25 & 10 & $x$ \\
\hline BEBC,7 & 5.8 & 13.4 & 28.7 & 6 & Partially \\
\hline BEBC, 8 & 22.4 & 57.9 & 158.0 & 5 & $\checkmark$ \\
\hline \multicolumn{6}{|c|}{ Data on deuterium } \\
\hline BEBC,9 & 14 & 2 & 4 & 8 & $\checkmark$ \\
\hline BEBC, 10 & 139 & 36 & 18 & 6 & $x$ \\
\hline Total for $\bar{\nu}_{\mu} p$ & 292 & 135 & 246 & 45 & \\
\hline \multicolumn{6}{|c|}{$\bar{\nu}_{\mu}+n \rightarrow \mu^{+} X^{-}$} \\
\hline BEBC, 11 & 6.9 & 6.7 & 16.9 & 8 & $\checkmark$ \\
\hline $\mathrm{BEBC}, 12$ & 61.6 & 44.5 & 82.9 & 6 & $x$ \\
\hline$\underline{\text { Total for } \bar{\nu}_{\mu} n}$ & 69 & 51 & 100 & 14 & \\
\hline \multicolumn{6}{|c|}{$\chi^{2}$ summary } \\
\hline All data & 2,398 & 1,068 & 731 & 154 & \\
\hline All ${ }^{2} \mathrm{H}$ data & 1,879 & 858 & 320 & 76 & \\
\hline All $\mathrm{H}$ data & 519 & 202 & 411 & 78 & \\
\hline
\end{tabular}

contribution to the total $\chi^{2}$ after the global tune are BEBC,3 and BEBC,5.

The main effect of the tune is observed in the PYTHIA region, at $W>3 \mathrm{GeV} / c^{2}$, where the prediction of $\left\langle n_{\mathrm{ch}}\right\rangle$ increased. This is a direct consequence of the increase on Lund $a$ and Lund $b$. This behavior is consistent with the HERMES tune, summarized in Sec. II B.

For each parameter, the corresponding uncertainty is obtained with the profiling method under the condition $\Delta \chi_{\text {profile }}^{2}\left(\theta_{i}\right)<1$. The profiles are calculated by fixing the value of the parameter under study $\theta_{i}$ to a desired value and minimizing the quantity $\Delta \chi^{2}(\boldsymbol{\theta})=\chi^{2}(\boldsymbol{\theta})-\chi_{\min }^{2}$ with respect to all others parameters that were allowed to float in the fit. The constant $\chi_{\min }^{2}$ corresponds to the global

TABLE X. Total $\chi^{2}$ calculated with the datasets included in each fit: 2010 GENIE, $\chi_{2010}^{2}$, 2021 GENIE, $\chi_{2021(\text { Global })}^{2}$, and 2021 GENIE, $\chi_{2021\left({ }^{2} \mathrm{H}\right)}^{2}$.

\begin{tabular}{lcccr}
\hline \hline Datasets & $\chi_{2010}^{2}$ & $\chi_{2021(\text { Global })}^{2}$ & $\chi_{2021\left({ }^{2} \mathrm{H}\right)}^{2}$ & $\begin{array}{c}\text { Degrees } \\
\text { of freedom }\end{array}$ \\
\hline All Data in tune & 486 & 242 & 410 & 109 \\
2H Data in tune & 230 & 105 & 37 & 52 \\
H Data in tune & 256 & 138 & 374 & 57 \\
\hline \hline
\end{tabular}




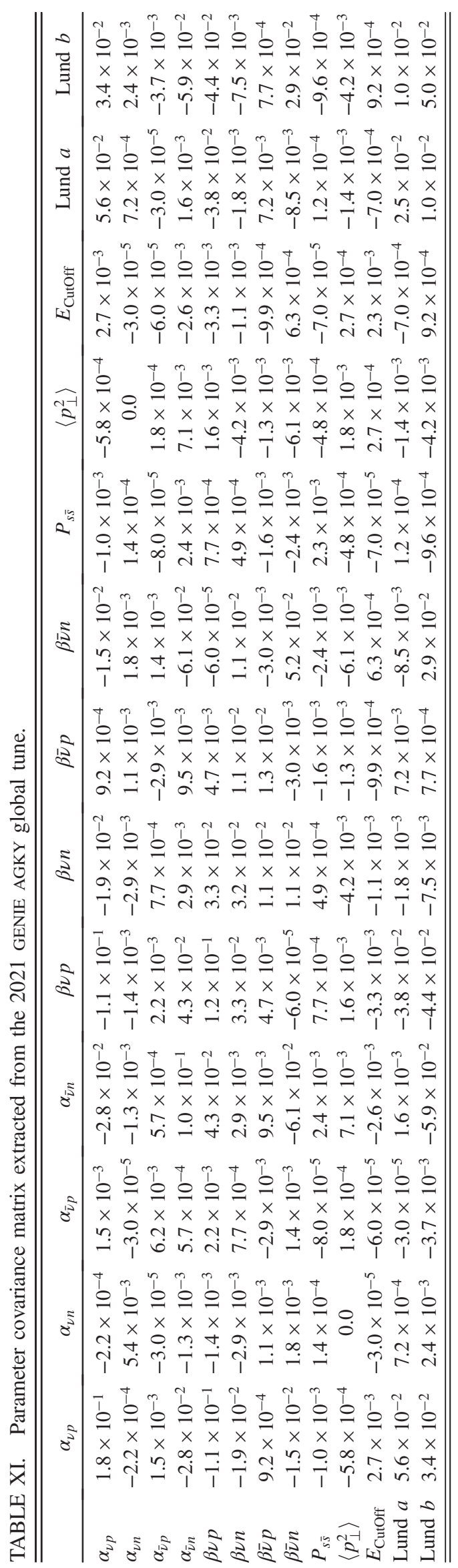




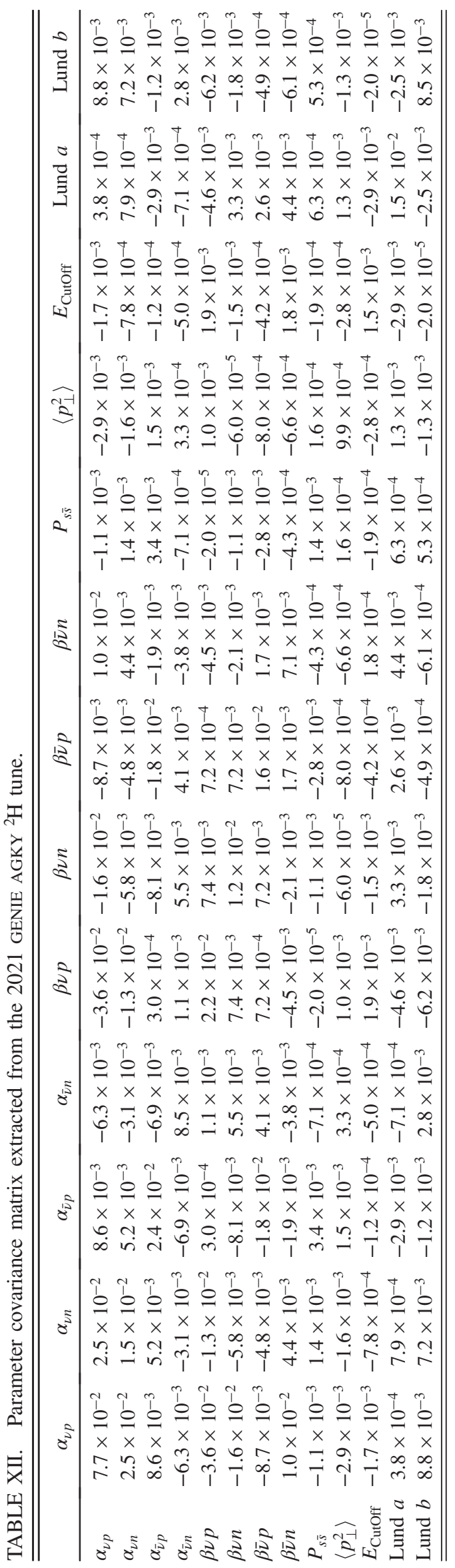




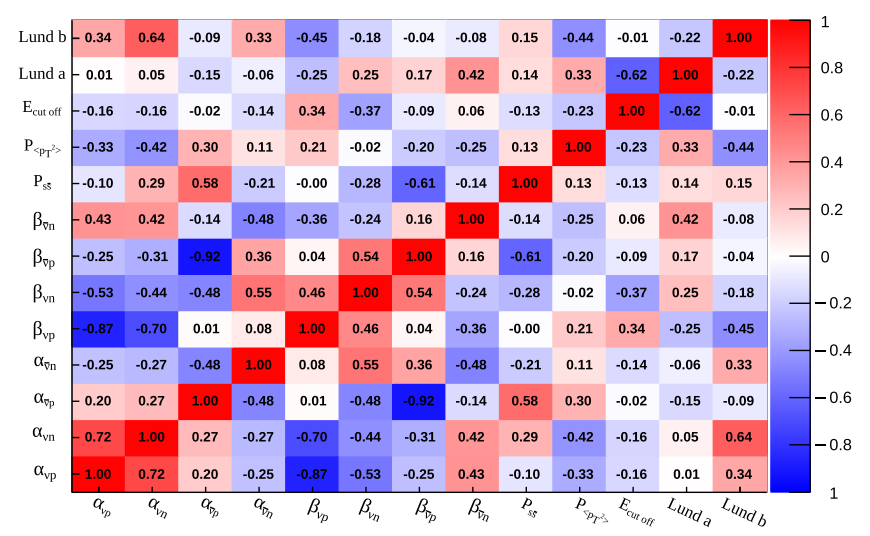

(a)

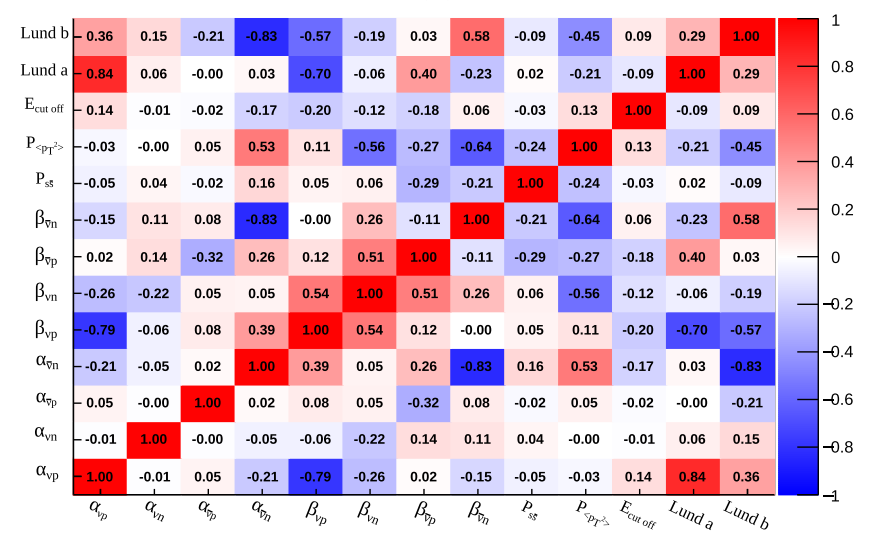

(b)

FIG. 17. Parameter correlation matrix for the 2021 GENIE AGKY tunes against averaged charged multiplicity data. (a) Global tune correlation matrix and (b) deuterium only tune correlation matrix. minimum value of $\chi^{2}(\boldsymbol{\theta})$. Some parameters have good Gaussian behavior and a symmetric profile. For some others, this is not true, and this gives rise to asymmetric uncertainties for the parameters. An example of a symmetric parameter profile compared to the non-Gaussian ones is shown in Fig. 18. The contours for some pairs of the AGKY parameters are shown in Fig. 19.

The fit covariance matrix can be propagated back to the GENIE predictions giving a posterior confidence belt for the prediction associated to the tune. As an example, a comparison of the global tune prediction and the associated posterior confidence belt is shown in Fig. 11.

\section{B. 2021 GENIE AGKY ${ }^{2} \mathrm{H}$ tune}

For the datasets included in the deuterium-only tune, the $\chi^{2}$ associated to the 2010 GENIE AGKY prediction is $\chi_{2010}^{2}=$ $230 / 52 \mathrm{DoF}$. After the tune, the total $\chi_{2021(\mathrm{H})}^{2}$ is $37 / 52 \mathrm{DoF}$ that corresponds to a $\mathrm{p}$ value of 0.94 . With the deuteriumonly goodness of fit being so much better than the global tune confirms the high tension between $\mathrm{H}$ and ${ }^{2} \mathrm{H}$ datasets.

Surprisingly, the deuterium-only tune shows better agreement than the global tune when all neutrino-induced hadronization data are considered; see Table IX. Yet, this does not imply that the deuterium-only fit is a better tune; it simply reinforces that the discarded dataset is not compatible with the data used in the fit.

The tension between hydrogen and deuterium data was already observed in other studies where a modified KNObased model was tuned to averaged charged multiplicity data from bubble chamber experiments [42]. Those studies suggest that the origin of tensions between $\mathrm{H}$ and ${ }^{2} \mathrm{H}$ could be due to rescattering effects on deuterium. As explained in Sec. III, the bubble chamber experiments claim that rescattering effects have a smaller effect on neutron

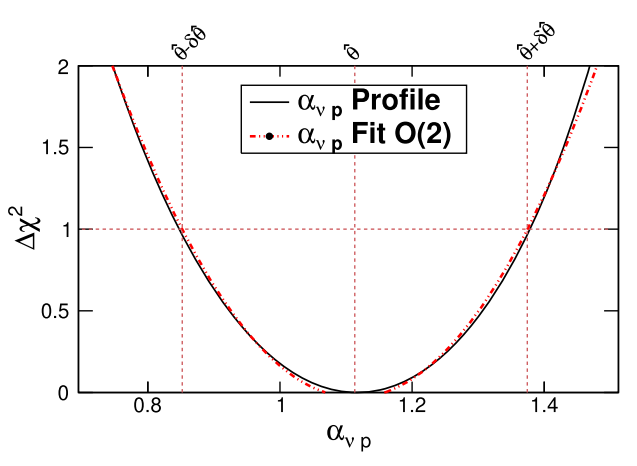

(a)
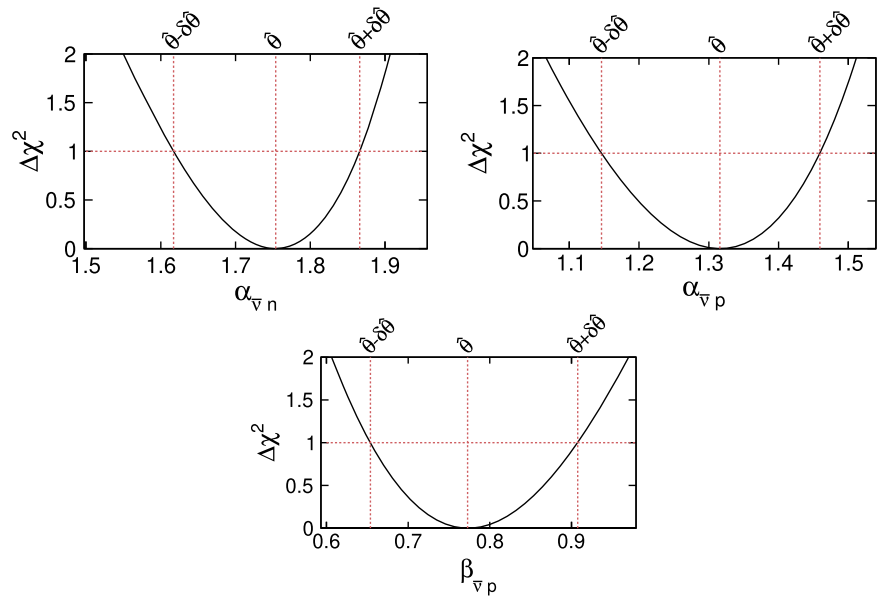

(b)

FIG. 18. Joint function obtained fixing the two parameters under study and minimizing $\Delta \chi_{\text {profile }}^{2}(\boldsymbol{\theta})$ with respect to the other parameters in the 2021 GENIE global tune. The dashed lines represent the parameter range that satisfies the condition $\Delta \chi_{\text {profile }}^{2}\left(\theta_{i}\right)<1$. This is also denoted as $\hat{\theta} \pm \delta \hat{\theta}$. (a) Symmetric parameter profile, (b) asymmetric parameter profiles. 

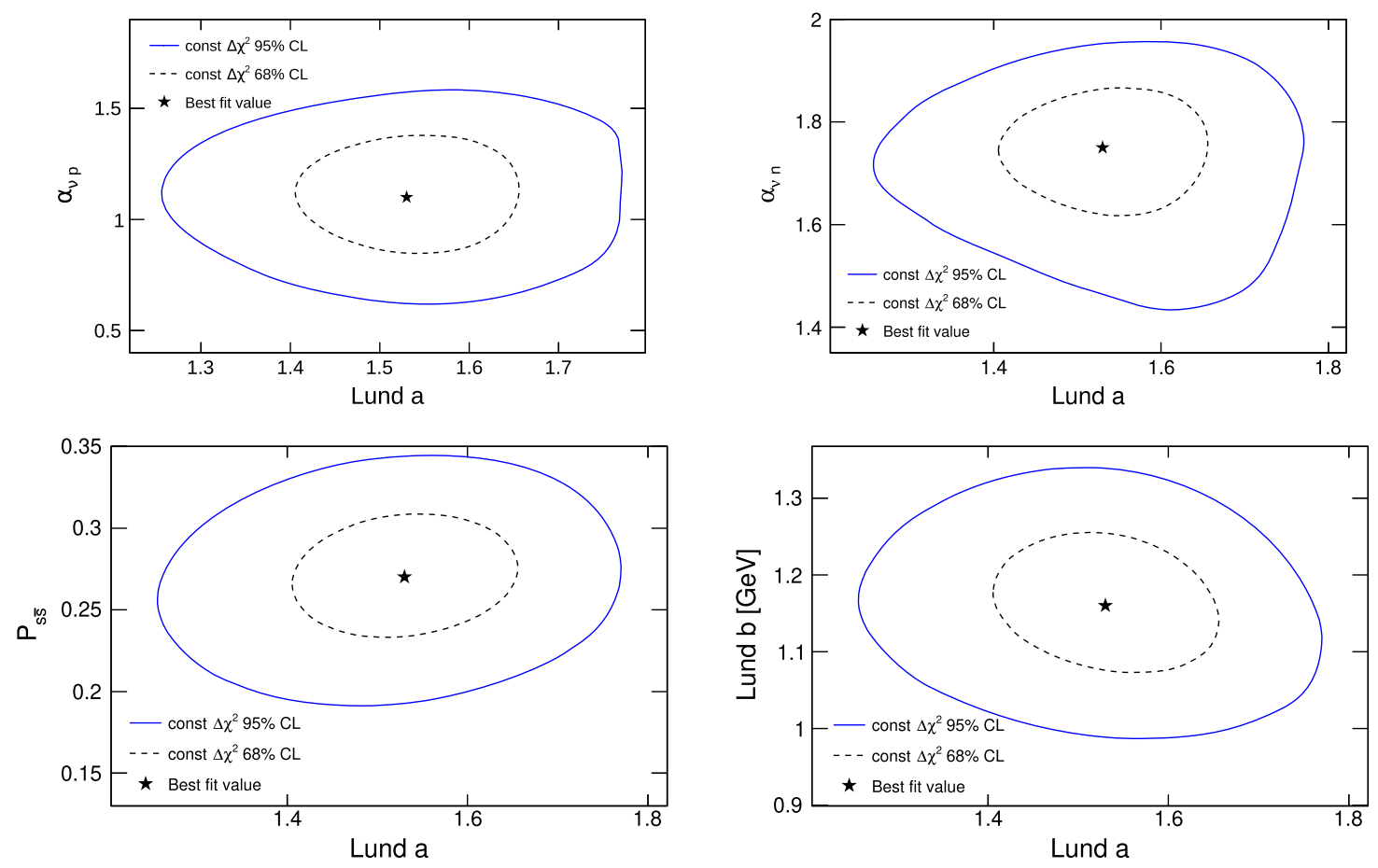

FIG. 19. Joint $\Delta \chi_{\text {contour }}^{2}\left(\theta_{i}, \theta_{j}\right)$ function obtained fixing the two parameters under study and minimizing $\Delta \chi_{\text {contour }}^{2}(\boldsymbol{\theta})$ respect the other parameters in the 2021 GENIE global tune. The $95 \%$ and $68 \%$ contour lines are shown as well as the best-fit values for the global tune.

samples as a consequence of the classification into $\nu_{\mu}$ on $p$ or $\nu_{\mu}$ on $n$ events. This is a consequence of the neutron reinteraction with the proton from the deuterium, which is then kicked out and therefore misidentified as a $\nu_{\mu}$ p event. If the disagreements were only due to rescattering, the global tune would have better agreement than the deuterium-only tune on $\nu_{\mu}$ and $\bar{\nu}_{\mu}$ on neutron data. However, better agreement of the global tune on neutron samples is not observed.

\section{AGKY global and deuterium-only tunes impact on other neutrino-induced hadronization observables}

The analyses procedure discussed in this paper focuses on the description of the charged averaged multiplicity. However, as discussed in Sec. IV A, different observables are linked with the shower particle content description. In this section, the effect of the 2021 global tune on different hadronization observables is discussed.

A wider comparison against all available hadronization observables for the G00_00a_00_00a AGKY predictions is reported in Ref. [27]. Some information provided by these observables is included in the tune using priors; see Sec. VI. The agreement of the 2021 GENIE AGKY global tune with these observables is not compromised.

There are, however, other observables that show tensions with the averaged charged multiplicity data. The neutral pion averaged multiplicity is related with the charged hadron multiplicity via Eq. (2); an increase on the charged averaged multiplicity is equivalent to a higher neutral pion averaged multiplicity. This result is incompatible with the data, as demonstrated in Fig. 20. Another example is the dispersion observable, defined as $D=\sqrt{\left\langle n^{2}\right\rangle-\langle n\rangle^{2}}$. The comparison of data on the ratio of $D /\left\langle n_{\mathrm{ch}}\right\rangle$ vs $W^{2}$ for the different tunes is shown in Fig. 21. In this case, the disagreement also increases with the invariant mass.

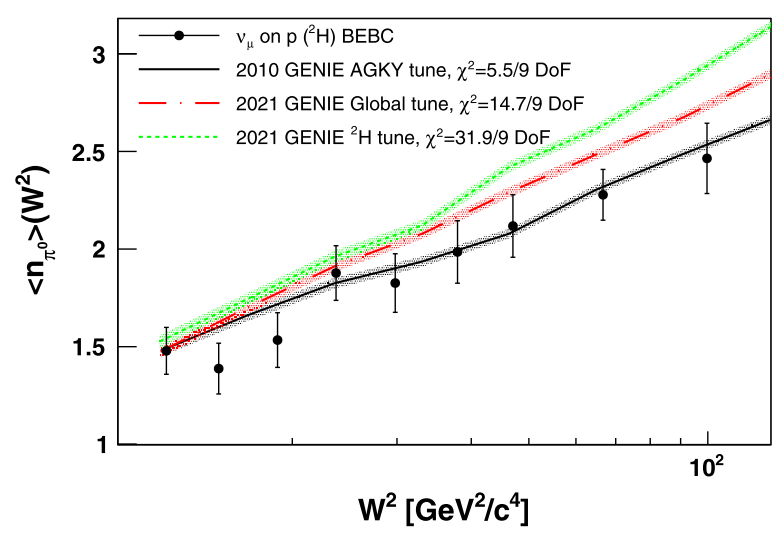

FIG. 20. Comparison of the predicted $\left\langle n_{\pi^{0}}\right\rangle$ against neutrinoinduced hadronization data on $\nu_{\mu}$ interactions on proton (p) from the BEBC bubble chamber experiment filled with ${ }^{2} \mathrm{H}[39,72]$. The predictions shown correspond to the 2010 GENIE AGKY (black), the 2021 GENIE AGKY global (red), and the 2021 GENIE AGKY ${ }^{2} \mathrm{H}$ (green) tunes. 


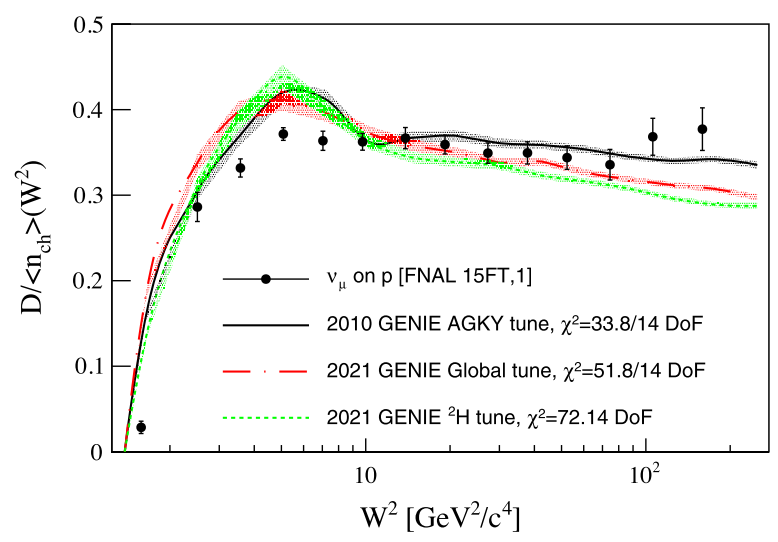

(a)

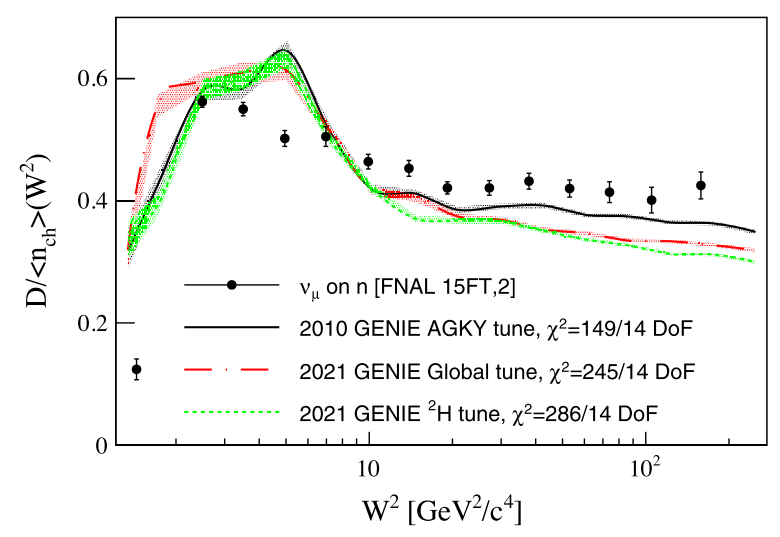

(b)

FIG. 21. Comparison of the predicted $D /\left\langle n_{\mathrm{ch}}\right\rangle$ against neutrino-induced hadrionzation data on $\nu_{\mu}$ interactions on proton (p) (a) and neutron (n) (b) from the FNAL $15 \mathrm{ft}$ bubble chamber experiment filled with ${ }^{2} \mathrm{H}$ [38]. The predictions shown correspond to the 2010 GENIE AGKY (black), the 2021 GENIE AGKY global (red), and the 2021 GENIE AGKY ${ }^{2} \mathrm{H}$ (green) tunes. (a) Comparison against $\nu_{\mu}$ on p data and (b) Comparison against $\nu_{\mu}$ on $\mathrm{n}$ data.

The tension between charged averaged multiplicity with $\left\langle n_{\pi^{0}}\right\rangle$ and dispersion data was already observed when using the HERMES parametrization described in Sec. IV B. The origin of these tensions is beyond the scope of this paper, as we aim for a better description of the charged averaged multiplicity data only. The further understanding of the connection between the different observables would require us to repeat the analyses procedure of this paper including other hadronizationrelated observables. Yet, it is important to understand how the 2021 GENIE AGKY global tune impacts other hadronization related observables.

\section{2021 GENIE AGKY global tune impact at the SIS region}

Other nonhadronization observables can be affected by this tune. The main impact is on the description of

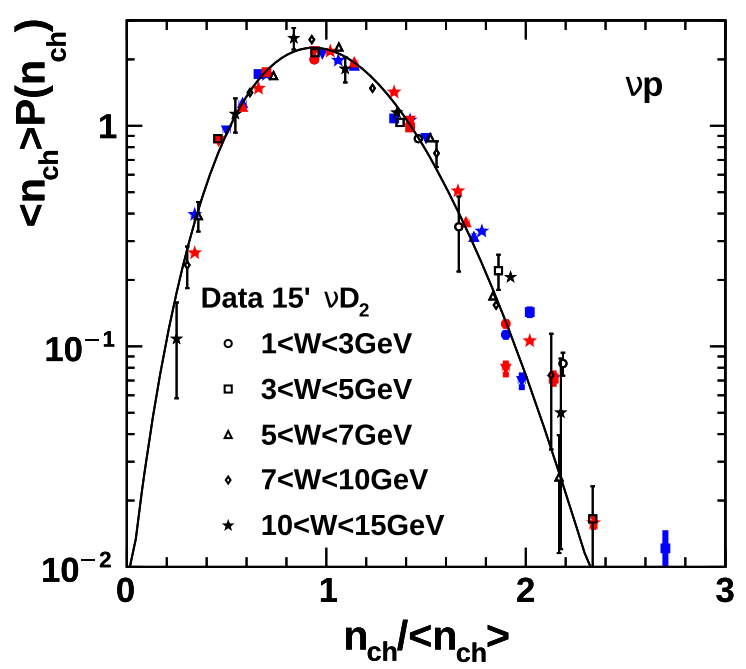

(a)

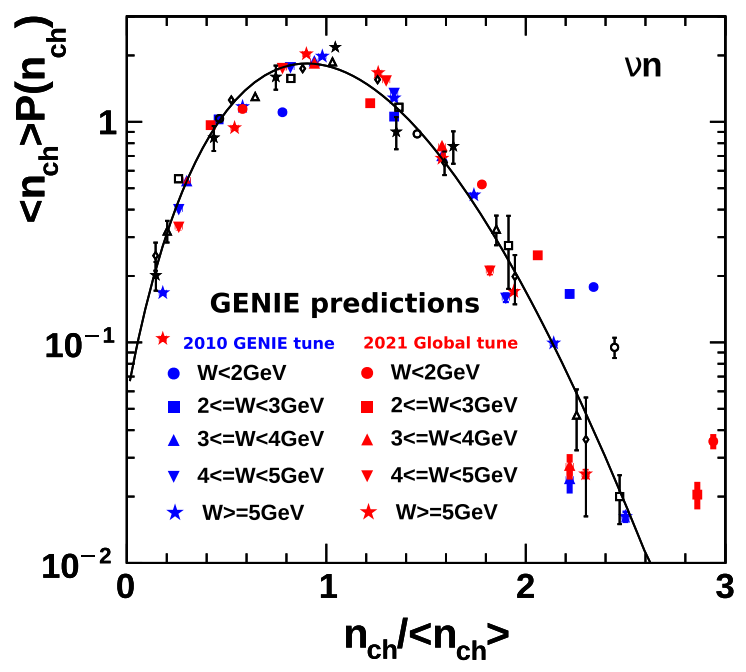

(b)

FIG. 22. Comparison of the KNO scaling distributions for neutrino interactions on deuterium against the predictions for 2010 GENIE tune (blue) and the 2021 GENIE global tune (red). The solid line is the best-fit result of the Levi function to FNAL $15 \mathrm{ft}$ bubble chamber data [38]. The $W$ range used for each data and predicted point is specified in the legend of Figs. 22 (a) and 22(b). (a) $\nu_{\mu} p$ KNO scaling distribution and (b) $\nu_{\mu} n \mathrm{KNO}$ scaling distribution.

the SIS region in GENIE, since it is linked with finalstate multiplicities [24]. In GENIE, the SIS is modeled applying scaling factors to the DIS cross section. These factors depend on the multiplicity of the process. Hence, variations on the final state multiplicity probabilities [Eq. (3)] change the scaling applied to the DIS cross section, affecting the DIS contribution to the SIS. The $P_{n}^{\text {had }}$ probability distributions for the 2010 GENIE AGKY tune and for the AGKY global tune are shown in Fig. 22. 


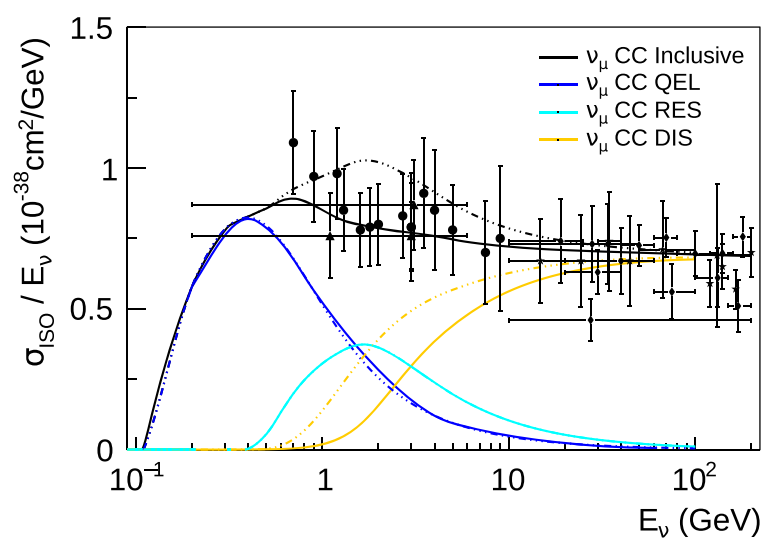

(a)

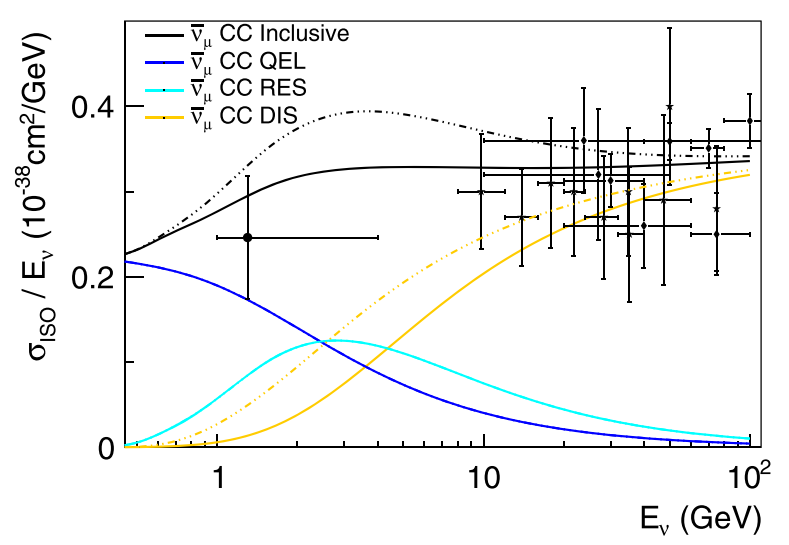

(b)

FIG. 23. Comparison of the $\nu_{\mu}$ and $\bar{\nu}_{\mu}$ CC inclusive cross section on free nucleon for the 2010 GENIE AGKY tune [24] (continuous lines) and the 2021 GENIE global tune (dashed lines) against hydrogen and deuterium data from Argonne National Lab (ANL) $12 \mathrm{ft}$ (triangle), Brookhaven National Lab (BNL) $7 \mathrm{ft}$ (filled circle), BEBC (diamond), and FNAL (filled star). The breakdown of the CC QEL, CC RES, and CC DIS contributions is shown for before and after the 2021 GENIE AGKY global tune. (a) $\nu_{\mu}$ CC inclusive cross section and (b) $\bar{\nu}_{\mu}$ CC inclusive cross section.

The impact of the AGKY tune on CC inclusive cross sections is summarized in Fig. 23. When applying the AGKY global tune to the SIS region, an increase of CC inclusive cross section is observed, for both $\nu_{\mu}$ and $\bar{\nu}_{\mu}$. The exclusive cross sections for different pion multiplicities show that the AGKY tune enhances the $2 \pi$ production while the $1 \pi$ production barely changes; see Fig. 24 . As a consequence, the agreement with inclusive and $\nu_{\mu} \mathrm{CC}$ $\pi^{+} \pi^{-}$data is lost.

Both the bare nucleon tune [24] and the 2021 GENIE global tune show a preference to increase the two-pion production, suggesting that a joint tune could preserve the agreement with inclusive and exclusive data at low $W$. This was neglected in previous analyses to minimize the tune's complexity, but this analysis clearly suggests otherwise. The high- $W$ AGKY parameters do not need

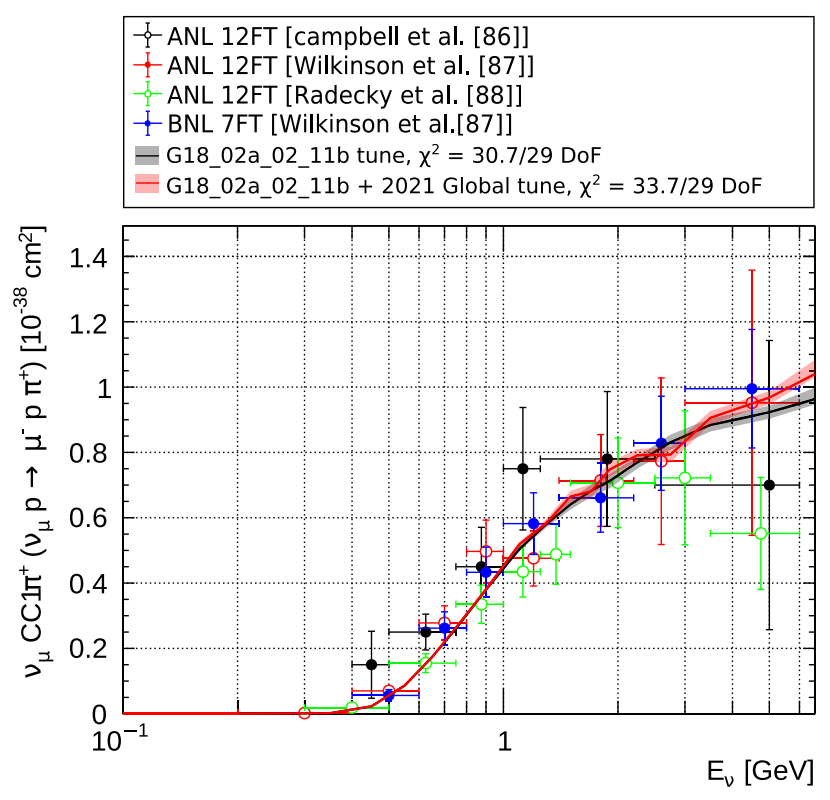

(a)

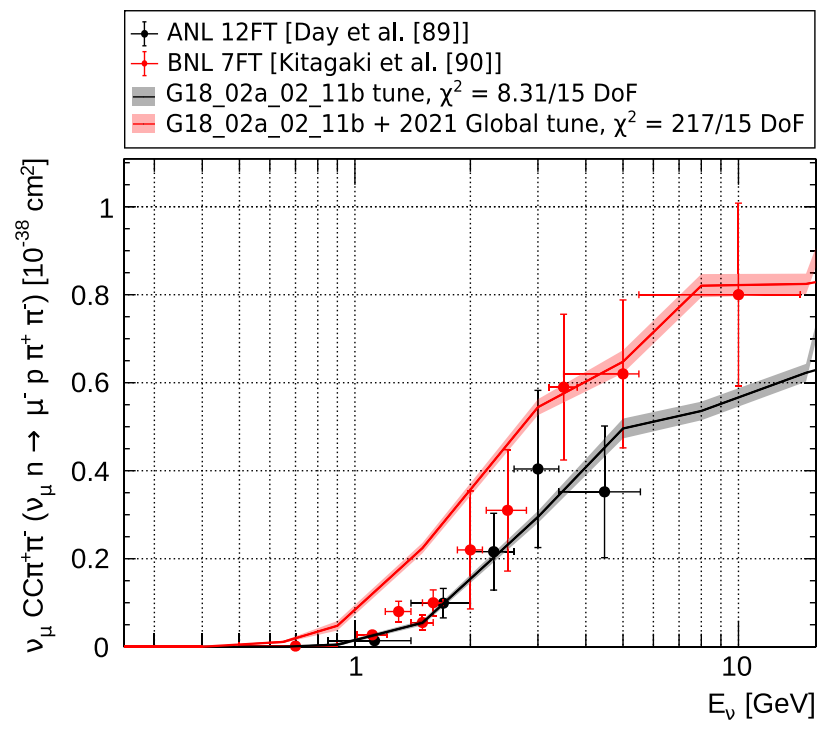

(b)

FIG. 24. Comparison of $\nu_{\mu} \mathrm{CC}$ exclusive cross section data on free nucleon for the 2010 GENIE AGKY tune [24] (black) and the 2021 GENIE global tune (red) against Argonne National Lab (ANL) $12 \mathrm{ft}$ and Brookhaven National Lab (BNL) $7 \mathrm{ft}$ data (See Refs. [86-90]). (a) One pion production and (b) two pion production.

anymore refinements. On the contrary, the low- $W$ parameters require a joint tune in order to have a satisfactory result that can be used to extract data-driven parameter uncertainties.

\section{CONCLUSIONS}

In this paper, we present the first GENIE tune of the AGKY model $[27,84]$, which was possible thanks to the Professor 
framework [85]. The analysis goal was to improve the GENIE agreement with neutrino charged averaged multiplicity data and to provide with the first data-driven constraints on hadronization parameters. Specifically, we constrained parameters of both the low- $W$ empirical model and PYTHIA using data from the BEBC and FNAL $15 \mathrm{ft}$ bubble chamber experiments filled with hydrogen and deuterium.

Tensions between hydrogen and deuterium data were observed, and two separate tunes were performed: a global and a deuterium-only tune. In particular, the 2021 AGKY global tune prediction underpredicts the deuterium data at the PYTHIA region, whereas the deuterium-only tune overpredicts the hydrogen data.

Further investigations on hadronization samples are needed in order to clarify the origin of this discrepancy. A possible solution could come from more recent neutrino experiments that released data on neutrino-induced hadronization. This is the case of NOMAD [91,92] for $\nu_{\mu}$ on mainly carbon target, CHORUS for $\nu_{\mu}$ and $\bar{\nu}_{\mu}$ on Fuji ET-7B emulsion [44,93], OPERA for $\nu_{\mu}$ on lead [43,94], and MicroBooNE for $\nu_{\mu}$ on argon [95]. But, of course, these samples include nuclear effects and therefore are not in the scope of this work.

Despite the tensions, the global tune shows better agreement with the charged averaged multiplicity data and provides the first data-driven analysis of this kind using neutrino interactions. This statistical analysis can be a useful input for proper systematic studies of modern neutrino experiments. The main effect of the tune is the increase of the averaged charged multiplicity for $W^{2}>10 \mathrm{GeV}^{2} / c^{4}$, modeled with PYTHIA. The low- $W$ region is also affected, but constraints due to energy, momentum, charge, baryon number, and strangeness conservation laws reduce the available phase space and the effect of the tuning procedure.

The effect of the 2021 GENIE AGKY global tune at the SIS region is an increase on the two-pion production cross section, which affects the current agreement with CC inclusive data [24]. Therefore, we conclude that this tune is more appropriate at higher energies where the contribution of the SIS region is not relevant. The information on the systematic uncertainties coming from the low- $W$ AGKY parameters is still valuable for neutrino experiments interested in the $W<2 \mathrm{GeV} / c^{2}$ region. A joint tune of the SIS region and hadronization datasets would address this disagreement.

\section{ACKNOWLEDGMENTS}

We would like to thank Andy Buckley (University of Glasgow, Scotland, U.K.) and Holger Schultz (Institute of Particle Physics Phenomenology, University of Durham, England, U.K.) for their support interfacing the Professor tool with the software products that underpin the GENIE global analyses. We would like to thank the CC-IN2P3
Computing Center as well as the Particle Physics Department at Rutherford Appleton Laboratory for providing computing resources and for their support. This work, as well as the ongoing development of several other GENIE physics tunes, was enabled through a Ph.D. studentship funded by STFC through the Liverpool Big Data Science Centre for Doctoral Training (Project No. 2021488). The initial conceptual and prototyping work for the development of the GENIE/Professor interfaces, as well as for the development of the GENIE global analyses framework that, currently, underpins several analyses, was supported in part through an Associateship Award by the Institute of Particle Physics Phenomenology, University of Durham. This document was prepared by the GENIE Collaboration using the resources of the Fermi National Accelerator Laboratory (Fermilab), a U.S. Department of Energy, Office of Science, HEP User Facility. Fermilab is managed by Fermi Research Alliance, LLC (FRA), acting under Contract No. DE-AC02-07CH11359.

\section{APPENDIX A: DATASET COMPATIBILITY STUDY}

The tensions highlighted in the paper were investigated to understand if their source could be caused by a specific dataset or analysis procedure. To do that, we performed a series of tunes using all the data used in the AGKY 2021 global fit, leaving one dataset out at a time. For each fit, we plotted the parameter best-fit value; see Fig. 25. Most of the partial fits results are compatible with the AGKY 2021 global tune predicted values. There are only two datasets in disagreement with the rest: BEBC,1 and BEBC.2 (hydrogen dataset) ${ }^{1}$ and FNAL, 1 (deuterium dataset). Those datasets use different target, they come from different experiments, and they were analyzed in different years. Hence, the cause of the tension cannot be due to a specific dataset or experiment.

\section{LIST OF ACRONYMS}

MC Monte Carlo

CC Charged-Current.

DIS Deep Inelastic Scattering.

SIS Shallow Inelastic Scattering.

FSI Final State Interactions.

KNO Koba-Nielsen-Olesen scaling law.

AGKY Andreopoulos-Gallagher-Kehayias-Yang.

BEBC Big European Bubble Chamber.

CMC Comprehensive Model Configurations.

EMI External Muon Identifier.

LPS Longitudinal Phase Space model.

\footnotetext{
${ }^{1}$ Please note that BEBC 1 is only one point, which is why we count it together with BEBC 2.
} 


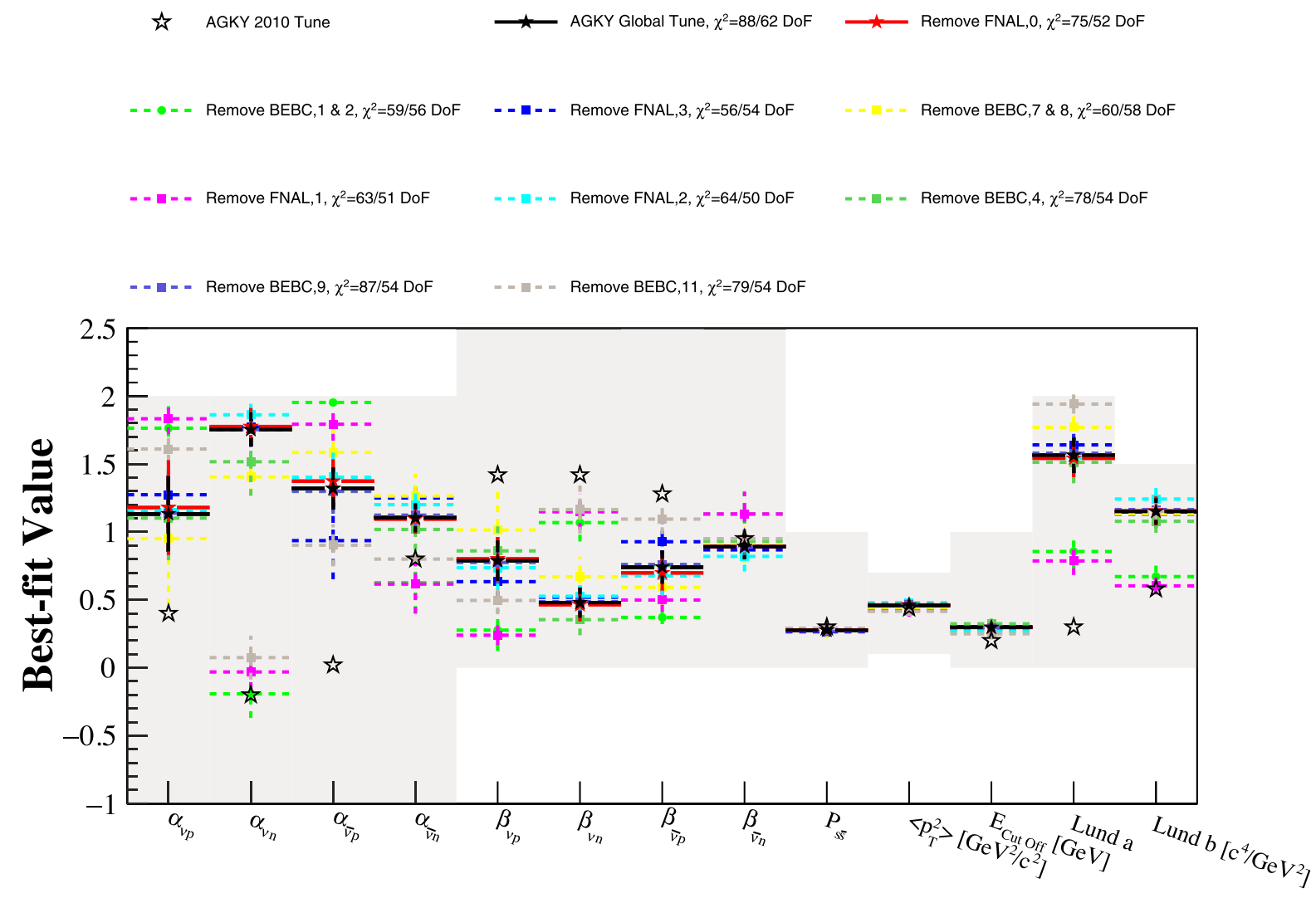

FIG. 25. Summary of best-fit values for a series of partial tunes obtained by using all the data from the AGKY 2021 global tune leaving one dataset out at a time. The result of the fit when removing a $\mathrm{H}$ dataset is shown in circles and when removing a ${ }^{2} \mathrm{H}$ dataset is shown in squares. The $\chi^{2}$ for each tune is listed in the legend. The gray area represents the available range for each parameter. The AGKY 2021 global tune best fit result is shown in filled black stars. The previous GENIE parameters are shown in empty stars (AGKY 2010 tune). The plotted errors are the square roots of the covariance diagonal elements.

[1] K. Abe et al. (T2K Collaboration), Observation of Electron Neutrino Appearance in a Muon Neutrino Beam, Phys. Rev. Lett. 112, 061802 (2014).

[2] S. Childress and J. Strait (NuMI, NOvA, LBNE Collaborations), Long baseline neutrino beams at fermilab, J. Phys. Conf. Ser. 408, 012007 (2013).

[3] L. Aliaga et al. (MINERvA Collaboration), Design, calibration, and performance of the MINER $\nu \mathrm{A}$ detector, Nucl. Instrum. Methods Phys. Res., Sect. A 743, 130 (2014).

[4] A. A. Aguilar-Arevalo et al. (MiniBooNE Collaboration), The MiniBooNE detector, Nucl. Instrum. Methods Phys. Res., Sect. A 599, 28 (2009).

[5] L. Alvarez-Ruso, Neutrino interactions: Challenges in the current theoretical picture, Nucl. phys., B Proc. suppl. 229-232, 167 (2012).

[6] L. Alvarez-Ruso, Y. Hayato, and J. Nieves, Progress and open questions in the physics of neutrino cross sections at intermediate energies, New J. Phys. 16, 075015 (2014).
[7] H. Gallagher, G. Garvey, and G. P. Zeller, Neutrino-nucleus interactions, Annu. Rev. Nucl. Part. Sci. 61, 355 (2011).

[8] L. Alvarez-Ruso, Y. Hayato, and J. Nieves, Progress and open questions in the physics of neutrino cross sections at intermediate energies, New J. Phys. 16, 075015 (2014).

[9] J. Nieves, R. Gran, I. Ruiz Simo, F. Sánchez, and M. J. Vicente Vacas, Neutrino-nucleus CCQE-like scattering, Nucl. Part. Phys., Proc. 273-275, 1830 (2016).

[10] A. A. Aguilar-Arevalo et al. (MiniBooNE Collaboration), First measurement of the muon neutrino charged current quasielastic double differential cross section, Phys. Rev. D 81, 092005 (2010).

[11] A. A. Aguilar-Arevalo et al. (MiniBooNE Collaboration), Measurement of Muon Neutrino Quasi-Elastic Scattering on Carbon, Phys. Rev. Lett. 100, 032301 (2008).

[12] K. Huang, Measurement of the neutrino-oxygen neutral current quasi-elastic interaction cross-section by observing nuclear de-excitation $\gamma$-rays in the $\mathrm{T} 2 \mathrm{~K}$ experiment, Ph.D. thesis, Kyoto University, 2015. 
[13] D. Ruterbories et al. (MINERvA Collaboration), Measurement of quasielastic-like neutrino scattering at $\left\langle E_{\nu}\right\rangle \sim$ $3.5 \mathrm{GeV}$ on a hydrocarbon target, Phys. Rev. D 99, 012004 (2019).

[14] K. Abe et al. (T2K Collaboration), Measurement of the $\nu_{\mu}$ charged current quasielastic cross section on carbon with the T2K on-axis neutrino beam, Phys. Rev. D 91, 112002 (2015).

[15] P. Abratenko et al. (MicroBooNE Collaboration), First Measurement of Differential Charged Current Quasielastic-like $\nu_{\mu}$-Argon Scattering Cross Sections with the MicroBooNE Detector, Phys. Rev. Lett. 125, 201803 (2020).

[16] P. Abratenko et al. (MicroBooNE Collaboration), Measurement of differential cross sections for $\nu_{\mu}$-Ar charged-current interactions with protons and no pions in the final state with the MicroBooNE detector, Phys. Rev. D 102, 112013 (2020).

[17] O. Lalakulich and U. Mosel, Pion production in the MiniBooNE experiment, Phys. Rev. C 87, 014602 (2013).

[18] K. Hiraide (SciBooNE Collaboration), Measurement of charged current charged single pion production in SciBooNE, in Proceedings of the 34th International Conference on High Energy Physics (ICHEP 2008), Philadelphia, Pennsylvania, USA, 2008 (2008), https://www.osti.gov/ biblio/940083-measurement-charged-current-charged-sing le-pion-production-sciboone.

[19] A. A. Aguilar-Arevalo et al. (MiniBooNE Collaboration), Measurement of neutrino-induced charged-current charged pion production cross sections on mineral oil at $E_{\nu} \sim 1 \mathrm{GeV}$, Phys. Rev. D 83, 052007 (2011).

[20] A. Rodriguez et al. (K2K Collaboration), Measurement of single charged pion production in the charged-current interactions of neutrinos in a $1.3 \mathrm{GeV}$ wide band beam, Phys. Rev. D 78, 032003 (2008).

[21] K. Abe et al. (T2K Collaboration), First measurement of the muon neutrino charged current single pion production cross section on water with the T2K near detector, Phys. Rev. D 95, 012010 (2017).

[22] R. Acciarri, C. Adams, J. Asaadi, B. Baller, T. Bolton, C. Bromberg et al. (The ArgoNeuT Collaboration), First measurement of the cross section for $\nu_{\mu}$ and $\bar{\nu}_{\mu}$ induced single charged pion production on argon using argoneut, Phys. Rev. D 98, 052002 (2018).

[23] C. Adams et al. (MicroBooNE Collaboration), First measurement of $\nu_{\mu}$ charged-current $\pi^{0}$ production on argon with the MicroBooNE detector, Phys. Rev. D 99, 091102 (2019).

[24] J. Tena-Vidal et al. (GENIE Collaboration), Neutrinonucleon cross-section model tuning in GENIE v3, Phys. Rev. D 104, 072009 (2021).

[25] P. Adamson et al. (MINOS Collaboration), Measurement of Neutrino Oscillations with the MINOS Detectors in the NuMI Beam, Phys. Rev. Lett. 101, 131802 (2008).

[26] C. Andreopoulos et al., The GENIE Neutrino Monte Carlo generator, Nucl. Instrum. Methods Phys. Res., Sect. A 614, 87 (2010).

[27] T. Yang, C. Andreopoulos, H. Gallagher, K. Hoffmann, and P. Kehayias, A hadronization model for few-GeV neutrino interactions, Eur. Phys. J. C 63, 1 (2009).
[28] T. Sjöstrand, S. Mrenna, and P.Z. Skands, PYTHIA 6.4 physics and manual, J. High Energy Phys. 05 (2006) 026.

[29] C. Adams et al. (LBNE Collaboration), The long-baseline neutrino experiment: Exploring fundamental symmetries of the Universe, in Proceedings of the Snowmass: Workshop on Energy Frontier, Seattle, Washington, USA, 2013 (2013), https://www.osti.gov/biblio/1128102.

[30] A. Ishihara, The IceCube upgrade-Design and science goals, Proc. Sci. ICRC2019(2021) 1031 [arXiv:1908.09441].

[31] D. Williams, Status and prospects for the IceCube neutrino observatory, Nucl. Instrum. Methods Phys. Res., Sect. A 952, 161650 (2020), 10th International Workshop on Ring Imaging Cherenkov Detectors (RICH 2018).

[32] A. Kouchner, Next-generation atmospheric neutrino experiments, Phys. Dark Universe 4, 60 (2014).

[33] M. Honda, M. S. Athar, T. Kajita, K. Kasahara, and S. Midorikawa, Atmospheric neutrino flux calculation using the NRLMSISE-00 atmospheric model, Phys. Rev. D 92, 023004 (2015).

[34] B. Abi et al. (DUNE Collaboration), Long-baseline neutrino oscillation physics potential of the DUNE experiment, Eur. Phys. J. C 80, 978 (2020).

[35] E. Valencia, D. Jena, Nuruzzaman, F. Akbar, L. Aliaga, D. Andrade, M. Ascencio, A. Bashyal, L. Bellantoni, A. Bercellie et al., Constraint of the MINER $\nu$ A medium energy neutrino flux using neutrino-electron elastic scattering, Phys. Rev. D 100, 092001 (2019).

[36] A. A. Aguilar-Arevalo, C. E. Anderson, A. O. Bazarko, S. J. Brice, B. C. Brown, L. Bugel, J. Cao, L. Coney, J. M. Conrad, D. C. Cox et al., Neutrino flux prediction at MiniBooNE, Phys. Rev. D 79, 072002 (2009).

[37] M. A. G. Aivazis, F. I. Olness, and W.-K. Tung, Leptoproduction of heavy quarks. I. general formalism and kinematics of charged current and neutral current production processes, Phys. Rev. D 50, 3085 (1994).

[38] D. Zieminska, S. Kunori, C. Y. Chang, G. A. Snow, D. Son, P. H. Steinberg et al., Charged-particle multiplicity distributions in $\nu n$ and $\nu p$ charged-current interactions, Phys. Rev. D 27, 47 (1983).

[39] S. Barlag et al. (Amsterdam-Bologna-Padova-PisaSaclay-Torino Collaboration), Charged hadron multiplicities in high energy $\bar{\nu}_{\mu} n$ and $\bar{\nu}_{\mu} p$ interactions, Z. Phys. C 11, 283 (1982); 14, 281(E) (1982).

[40] M. Sakuda, Results from low-energy neutrino-nucleus scattering experiments, Nucl. phys., B Proc. suppl. 112, 109 (2002).

[41] T. Katori and S. Mandalia, PYTHIA hadronization process tuning in the GENIE neutrino interaction generator, J. Phys. G 42, 115004 (2015).

[42] K. S. Kuzmin and V. A. Naumov, Mean charged multiplicities in charged-current neutrino scattering on hydrogen and deuterium, Phys. Rev. C 88, 065501 (2013).

[43] N. Agafonova et al. (OPERA Collaboration), Study of charged hadron multiplicities in charged-current neutrinolead interactions in the OPERA detector, Eur. Phys. J. C 78, 62 (2018); 78, 747(E) (2018).

[44] A. Kayis-Topaksu et al. (CHORUS Collaboration), Charged particle multiplicities in charged-current neutrino-and antineutrino-nucleus interactions, Eur. Phys. J. C 51, 775 (2007). 
[45] W. Wittek et al. (BEBC WA59 Collaboration), Production of $\pi^{0}$ mesons and charged hadrons in $\bar{\nu}$ neon and $\nu$ neon charged current interactions, Z. Phys. C 40, 231 (1988).

[46] Z. Koba, H. B. Nielsen, and P. Olesen, Scaling of multiplicity distributions in high-energy hadron collisions, Nucl. Phys. B40, 317 (1972).

[47] T. Sjöstrand, S. Mrenna, and P.Z. Skands, A brief introduction to PYTHIA 8.1, Comput. Phys. Commun. 178, 852 (2008).

[48] J. G. Rubin, Polarization, motion, and fragmentation: Exploring the role of quarks in the nucleon through semi-inclusive longitudinal spin asymmetries at HERMES, $\mathrm{Ph} . \mathrm{D}$. thesis, University of Illinois, 2009.

[49] S. Ferreres-Solé and T. Sjöstrand, The space-time structure of hadronization in the Lund model, Eur. Phys. J. C 78, 983 (2018).

[50] J. M. Katzy, QCD Monte-Carlo model tunes for the LHC, Prog. Part. Nucl. Phys. 73, 141 (2013).

[51] P. Z. Skands, Tuning Monte Carlo generators: The Perugia tunes, Phys. Rev. D 82, 074018 (2010).

[52] R. Corke and T. Sjöstrand, Interleaved parton showers and tuning prospects, J. High Energy Phys. 03 (2011) 032.

[53] G. Aad et al. (ATLAS Collaboration), Charged-particle multiplicities in $p p$ interactions measured with the ATLAS detector at the LHC, New J. Phys. 13, 053033 (2011).

[54] ATLAS Collaboration, New ATLAS event generator tunes to 2010 data, Report No. ATL-PHYS-PUB-2011-008, 2011.

[55] ATLAS Collaboration, ATLAS tunes of PYTHIA 6 and PYTHIA 8 for MC11, Report No. ATL-PHYS-PUB-2011009, 2011.

[56] ATLAS Collaboration, Summary of ATLAS PYTHIA 8 tunes , Report No. ATL-PHYS-PUB-2012-003, 2012.

[57] R. Field, The underlying event in hadronic collisions, Annu. Rev. Nucl. Part. Sci. 62, 453 (2012).

[58] J. Alcaraz Maestre et al. (SM and NLO MULTILEG Working Group, SM MC Working Group), The SM and NLO Multileg and SM MC Working Groups: Summary Report, in Proceedings of the 7th Les Houches Workshop on Physics at TeV Colliders, Les Houches, France, 2011 (2012), pp. 1-220, https://inspirehep.net/literature/1282676.

[59] N. Firdous and G. Rudolph, Tuning of PYTHIA 6 to minimum bias data, Eur. Phys. J. Web Conf. 60, 20056 (2013).

[60] T. Sjöstrand, PYTHIA tunes (2014), http://home.thep.lu.se/ torbjorn/pythia81html/Tunes.html.

[61] P. Skands, S. Carrazza, and J. Rojo, Tuning PYTHIA 8.1: The Monash 2013 Tune, Eur. Phys. J. C 74, 3024 (2014).

[62] A. Airapetian et al. (HERMES Collaboration), Multiplicities of charged pions and kaons from semi-inclusive deepinelastic scattering by the proton and the deuteron, Phys. Rev. D 87, 074029 (2013).

[63] N. M. Agababyan, V. V. Ammosov, N. Grigoryan, H. Gulkanyan, A. A. Ivanilov, Z. Karamyan, and V. A. Korotkov, Charged $\rho$-meson production in neutrino induced reactions at $\left\langle E_{\nu}\right\rangle \approx 10 \mathrm{GeV}$, Yad. Fiz. 74, 238 (2011) [Phys. At. Nucl. 74, $221(2011)]$.

[64] J. W. Chapman et al., Multiplicity Distributions in HighEnergy Neutrino Interactions, Phys. Rev. Lett. 36, 124 (1976).
[65] H. Grassler et al. (Aachen-Birmingham-BonnCERN-Imperial College-München (MPI) - Oxford Collaboration), Multiplicities of secondary hadrons produced in $\nu p$ and $\bar{\nu} p$ charged current interactions, Nucl. Phys. B223, 269 (1983).

[66] G. T. Jones et al. (Birmingham-CERN-Imperial College-München (MPI)—Oxford-University College London (WA21) Collaboration), $W^{2}$ and $Q^{2}$ dependence of charged hadron and pion multiplicities in $\nu p$ and $\bar{\nu} p$ charged current interactions, Z. Phys. C 46, 25 (1990).

[67] G. T. Jones et al. (Birmingham-CERN-Imperial College-München (MPI)_Oxford-University College London (WA21) Collaboration), Multiplicity distributions of charged hadrons in $\nu p$ and $\bar{\nu} p$ charged current interactions, Z. Phys. C 54, 45 (1992).

[68] M. Derrick, P. Gregory, F. LoPinto, B. Musgrave, J. Schlereth, P. Schreiner et al., Multiplicity distributions in $\bar{\nu}_{\mu} p$ interactions, Phys. Rev. D 25, 624 (1982).

[69] J. Hanlon, R. A. Burnstein, H. A. Rubin, C. Y. Chang, T. Dombeck, G. A. Snow et al., Comparison of $\nu n$ and $\nu p$ Charged-Current Cross Sections from High-Energy Neutrino interactions in deuterium, Phys. Rev. Lett. 45, 1817 (1980).

[70] V. E. Barnes, T. S. Carman, D. D. Carmony, E. Fernandez, A. F. Garfinkel, A. T. Laasanen et al., Measurement of quark momentum distributions in the proton using an antineutrino probe, Phys. Rev. D 25, 1 (1982).

[71] J. Blietschau et al. (Aachen-Bonn-CERN-Munich (MPI)_Oxford Collaboration), Observation of scaling deviations in the energy distribution of secondary hadrons in inelastic neutrino-proton interactions, Phys. Lett. 87B, 281 (1979).

[72] B. Jongejans et al. (Amsterdam-Bergen-BolognaPadova-Pisa-Saclay-Torino (WA 25) Collaboration), Multiplicity distributions of charged hadrons produced in (anti)neutrino-deuterium charged- and neutral-current interactions, Nuovo Cimento A 101, 435 (1989).

[73] D. Allasia et al. (Amsterdam-Bergen-BolognaPadova-Pisa-Saclay-Torino Collaboration), Fragmentation in neutrino and antineutrino charged current interactions on proton and neutron, Z. Phys. C 24, 119 (1984).

[74] C. C. Chang, W. A. Mann, A. Napier, J. Schneps, R. A. Burnstein, J. Hanlon et al., Study of diquark fragmentation into $\Lambda$ and $Y^{*+}$ in $\nu n$ and $\nu p$ interactions, Phys. Rev. D 27, 2776 (1983).

[75] J. Bell, C. T. Coffin, R. N. Diamond, H. T. French, W. C. Louis, B.P. Roe et al., Experimental study of hadrons produced in high-energy charged-current neutrino-proton interactions, Phys. Rev. D 19, 1 (1979).

[76] A. Kayis-Topaksu et al. (CHORUS Collaboration), Charged-particle multiplicities in charged-current neutrinoand anti-neutrino-nucleus interactions, Eur. Phys. J. C 51, 775 (2007).

[77] A. Bodek and J. L. Ritchie, Fermi motion effects in deep inelastic lepton scattering from nuclear targets, Phys. Rev. D 23, 1070 (1981).

[78] C. Berger and L. M. Sehgal, Lepton mass effects in single pion production by neutrinos, Phys. Rev. D 76, 113004 (2007); 77, 059901(E) (2008).

[79] C. H. Llewellyn Smith, Neutrino reactions at accelerator energies, Phys. Rep. 3C, 261 (1972). 
[80] S. G. Kovalenko, Quasielastic neutrino production of charmed baryons from the point of view of local duality, Yad. Fiz. 52, 1478 (1990) [Sov. J. Nucl. Phys. 52, 934 (1990)].

[81] A. Pais, Weak interactions at high energies, Ann. Phys. (N.Y.) 63, 361 (1971).

[82] D. Rein and L. M. Sehgal, Neutrino-excitation of baryon resonances and single pion production, Ann. Phys. (N.Y.) 133, 79 (1981).

[83] M. A. G. Aivazis, W.-K. Tung, and F. I. Olness, Next-toleading order QCD formulation of deep inelastic scattering, in Proceedings of the Vancouver Meeting, Particles \& Fields'91: Vancouver, Canada, 1991 (World Scientific, River Edge,1991), pp. 663-665, https://inspirehep.net/ literature/326997.

[84] C. Andreopoulos et al., The GENIE neutrino Monte Carlo generator: Physics and user manual, arXiv:1510.05494.

[85] A. Buckley, H. Hoeth, H. Lacker, H. Schulz, and J. E. von Seggern, Systematic event generator tuning for the LHC, Eur. Phys. J. C 65, 331 (2010).

[86] J. Campbell et al., Study of the Reaction $\nu p \rightarrow \mu^{-} \pi^{+} p$, Phys. Rev. Lett. 30, 335 (1973).

[87] C. Wilkinson, P. Rodrigues, S. Cartwright, L. Thompson, and K. McFarland, Reanalysis of bubble chamber measurements of muon-neutrino induced single pion production, Phys. Rev. D 90, 112017 (2014).
[88] G. M. Radecky et al., Study of single-pion production by weak charged currents in low-energy $\nu d$ interactions, Phys. Rev. D 25, 1161 (1982).

[89] D. Day et al., Study of $\nu d$ charged-current two-pion production in the threshold region, Phys. Rev. D 28, 2714 (1983).

[90] T. Kitagaki et al., Charged-current exclusive pion production in neutrino-deuterium interactions, Phys. Rev. D 34, 2554 (1986).

[91] F. Vannucci, The NOMAD experiment at CERN, Adv. High Energy Phys. 2014, 1 (2014).

[92] A. Chukanov and R. Petti, Study of fragmentation parameters in deep inelastic scattering neutrino interactions, JPS Conf. Proc. 12, 010026 (2016).

[93] E. Radicioni (CHORUS Collaboration), Results from the CHORUS experiment at CERN, Phys. Rep. 85, 95 (2000).

[94] G. Giacomelli and M. Giorgini (OPERA Collaboration), The OPERA experiment, in Proceedings of the Vulcano Workshop: Frontier Objects in Astrophysics and Particle Physics, Vulcano, Italy, 2006 (2006), https://inspirehep.net/ literature/725806.

[95] C. Adams et al. (MicroBooNE Collaboration), Comparison of $\nu_{\mu}$-Ar multiplicity distributions observed by MicroBooNE to GENIE model predictions, Eur. Phys. J. C 79, 248 (2019). 